\title{
Elementary abelian 2-subgroups of Sidki-type in finite groups
}

\author{
Michael Aschbacherr, Robert Guralnick ${ }^{2}$ and Yoav Segev ${ }^{3}$ \\ Dedicated to Avinoam Mann on the occasion of his retirement, 2006
}

\begin{abstract}
Let $G$ be a finite group. We say that a nontrivial elementary abelian 2-subgroup $V$ of $G$ is of Sidki-type in $G$, if for each involution $i$ in $G, C_{V}(i) \neq 1$. A conjecture due to S. Sidki (J. Algebra 39, 1976) asserts that if $V$ is of Sidki-type in $G$, then $V \cap O_{2}(G) \neq 1$. In this paper we prove a stronger version of Sidki's conjecture. As part of the proof, we also establish weak versions of the saturation results of G. Seitz (Invent. Math. 141, 2000) for involutions in finite groups of Lie type in characteristic 2. Seitz's results apply to elements of order $p$ in groups of Lie type in characteristic $p$, but only when $p$ is a good prime, and 2 is usually not a good prime.
\end{abstract}

Mathematics Subject Classification (2000). Primary 20D05; Secondary 20D06, 20 D08.

Keywords. Finite simple groups, involutions, parabolic subgroups, fundamental subgroups, saturation.

\section{Introduction}

In the conference honoring the retirement of A. Mann which took place in Jerusalem in May 2006, Said Sidki gave a talk in which he recalled a conjecture that he had made in 1976 ([Si]). In this paper we prove his conjecture:

Theorem 1. Assume $G$ is a finite group and $V$ is a non-trivial elementary abelian 2subgroup of $G$ such that for each involution $i \in G, C_{V}(i) \neq 1$. Then $V \cap O_{2}(G) \neq 1$.

One way to view Theorem 1 is the following. For a finite group $G \operatorname{let} \operatorname{Inv}(G)$ be the set of involutions of $G$ and let $d$ be the commuting graph on $\operatorname{Inv}(G)$. Thus the vertex set of $d$ is $\operatorname{Inv}(G)$ with $a, b \in \operatorname{Inv}(G)$ adjacent in $d$ if $a$ and $b$ commute. Now to

\footnotetext{
${ }^{1}$ Partially supported by NSF-0504852.

${ }^{2}$ Partially supported by NSF-0140578.

${ }^{3}$ Partially supported by BSF grant no. 2004-083.
} 
any graph one can associate a combinatorial simplicial complex: the clique complex, whose simplices are the cliques of the graph. Let $K_{2}(G)$ be the clique complex of $d$. Then Theorem 1 says that if there exists a simplex $\sigma$ in $K_{2}(G)$ such that each vertex in $d$ is adjacent to some vertex of $\sigma$, then $\langle\sigma\rangle \cap O_{2}(G) \neq 1$.

Since the clique complex $K_{2}(G)$ is homotopy equivalent to the Quillen complex $\mathcal{A}_{2}(G)$ of $G$ at the prime 2 (cf. 5.2 in [A7]), Theorem 1 sheds some light on the Quillen conjecture which asserts that for any prime $p, \mathcal{A}_{p}(G)$ is contractible if and only if $O_{p}(G) \neq 1$. Indeed, Theorem 1 shows that the Quillen conjecture at the prime 2 is equivalent to the following statement: $K_{2}(G)$ is contractible iff $G$ possesses a subgroup of Sidki type.

Since the hypothesis of Theorem 1 does not inherit well to homomorphic images, we prove that $V \cap O_{2}(G) \neq 1$ under a weaker hypothesis on $V$ :

Theorem 2. Assume that $G$ is a finite group and $V$ is a nontrivial elementary abelian 2-subgroup of $G$, such that whenever $v \in V$ inverts an element $h$ of odd order in $G$ then $C_{V}(h) \neq 1$. Then $V \cap O_{2}(G) \neq 1$.

Notice that if $h$ is an element of odd order inverted by $v \in V^{\#}$ then $h v$ is an involution in $G$, and $C_{V}(h v) \neq 1$ iff $C_{V}(h) \neq 1$. Thus if $V$ satisfies the hypothesis of Theorem 1, then it also satisfies the hypothesis of Theorem 2; consequently Theorem 2 implies Theorem 1.

In [Al], Alperin proved Theorem 1 for $|V| \leq 4$; indeed in this case a short and elementary argument (Lemma 1.4) shows that Theorem 2 holds, but when $|V|>4$ we know of no such argument to establish Theorem 2. The case $|V|=8$ is interesting in that Theorem 1 was proved by Sidki in [Si] when $|V|=8$ using elementary means.

For the general case we show in Lemma 1.1 that if $(G, V)$ satisfies the hypothesis of Theorem 2, and $K \unlhd G$ with $V \cap K=1$, then $(G / K, V K / K)$ also satisfies this hypothesis. This allows us to reduce the proof of Theorem 2 to the case where $G$ is almost simple (Proposition 1.14). Then we appeal to the classification of the finite simple groups and results on the subgroup structure of such groups to complete the proof.

Call a pair $(G, V)$ that satisfies the hypothesis of Theorem 2 a Sidki pair. Let $(G, V)$ be a Sidki pair and suppose $v \in V^{\#}$ inverts an element $x \in G$ of odd order. Set $D:=\langle v, x\rangle$ and $C:=C_{G}(D)$. We observe that

(O1) $(H, V)$ is a Sidki pair for all subgroups $H \leq G$ with $V \leq H$ (obvious);

(O2) $(C, V \cap C)$ is a Sidki pair (Lemma 1.7);

(O3) if $(G, V)$ is a minimal counter example to Theorem 2, and $O_{2}(C)$ contains a unique involution $t$, then $t$ is contained in $V$ and $\left\langle t^{C_{G}(v)}\right\rangle$ is elementary abelian (Lemma 1.15(2)).

These three observations are very useful in the proof of Theorem 2. We now describe this proof in more detail. Suppose $(G, V)$ is a minimal counter example to Theorem 2. 
Then $G$ is an almost simple finite group; that is $L=F^{*}(G)$ is a nonabelian finite simple group. By the classification of the finite simple groups, $L$ is an alternating group, a finite group of Lie type, or one of 26 sporadic groups.

An easy argument (Theorem 2.1) shows that $L$ is not an alternating group. In Sections 3-6 we establish various results about strongly real elements in the automorphism groups of sporadic groups and use observations (O1)-(O3) above to show that $L$ is not sporadic.

This reduces us to the case where $L$ is a group of Lie type and characteristic $p$ for some prime $p$. We consider two subcases: $p$ is odd and $p$ is even.

In the odd characteristic case we employ a different strategy: assume $X$ is an almost simple finite group such that $F^{*}(X)$ is a group of Lie type and odd characteristic. Let $W$ be a nontrivial elementary abelian 2-subgroup of $X$. Recall that proper parabolic subgroups $P$ and $Q$ in $F^{*}(X)$ are called opposite if $P \cap Q$ is a Levi factor of both $P$ and $Q$. Let us denote by $\delta(X, W)$ the collection of those $W$-invariant sets $S$ of proper parabolic subgroups of $F^{*}(X)$ such that if $P, Q \in S$ are distinct, then $P$ and $Q$ are opposites.

In Lemma 8.1 we show that, in a minimal counter example to Theorem 2 , for all $P \in S \in \mathcal{S}(G, V), N_{V}(P)=1$. Thus to eliminate the possibility that $L$ is of Lie type in odd characteristic it suffices to establish the following result, which may be of independent interest:

Theorem 3. Assume $X$ is an almost simple finite group such that $F^{*}(X)$ is a group of Lie type and odd characteristic. Assume $W$ is a nontrivial elementary abelian 2-subgroup of $X$. Then:

(1) $\delta(X, W)$ is nonempty.

(2) If $|W| \geq 8$, then there exists $P \in S \in \mathcal{S}(X, W)$ such that $N_{W}(P) \neq 1$.

This reduces the proof of Theorem 2 to the case where $L$ is of Lie type and characteristic 2 . In this case we use observation $(\mathrm{O} 1)$ and the fact that $|V|>4$ to "force" certain involutions $v$ of $G$ to belong to $V$ and then we (essentially) argue that for some element $x \in G$ of odd order inverted by $v, D:=\langle v, x\rangle$ does not satisfy observation $(\mathrm{O} 2)$ or $(\mathrm{O} 3)$. This is done by showing that certain involutions in $L$ satisfy a weak version of the saturation properties established by Seitz in [Se] for elements of order $p$ in groups of Lie type and characteristic $p$.

Unfortunately the results of Seitz only apply when $p$ is a good prime, which is almost never the case for $p=2$. Thus we must supply proofs of these weak saturation properties ourselves. These properties are of independent interest and their description is somewhat technical; we first define the set $\mathfrak{S}(Y, i)$.

Let $q$ be a power of 2, and assume that $Y$ is a group such that either

$Y=O^{2^{\prime}}(Y)$ is a group of Lie type over $\mathbb{F}_{q}$ with $F^{*}(Y)$ quasisimple, or $Y={ }^{2} F_{4}(2)^{\prime}$ is the Tits group. 
Let $i \in \operatorname{Inv}(Y)$, and define $\mathfrak{S}(Y, i)$ to be the set of pairs $(K, U)$ such that $i \in U \leq K \leq Y$;

$U$ is an elementary abelian 2-group of order $q$ and $U \leq Z\left(C_{Y}(i)\right)$; $C_{Y}(K)$ is a complement to $O_{2}\left(C_{Y}(i)\right)$ in $C_{Y}(i)$;

Furthermore one of the following holds:

(i) $K \cong L_{2}(q)$;

(ii) $Y \cong S z(q)$ or ${ }^{2} F_{4}(q)$ with $q>2$, or ${ }^{2} F_{4}(2)^{\prime}, i$ is a long root involution in $Y$, and $K \cong S z(q), S z(q)$, or $D_{10}$, respectively;

(iii) $Y \cong \operatorname{Sp}_{n}(q)$ or $Y \cong F_{4}(q), i$ is of type $c_{2}$, respectively of type $t u$ in $Y$, and $K \cong L_{2}\left(q^{2}\right)$; in addition, if $q=2$, then $C_{Y}(K)=C_{Y}(D)$ for each dihedral subgroup $D_{10} \cong D$ of $K$.

In this definition, and in Theorem 4 below, we use the notation and the description of involutions in groups of Lie type and characteristic 2 from [ASe].

When $Y^{\prime}$ is of Lie type and characteristic $p$ with $p$ a good prime, Seitz [Se] establishes many properties of elements $i^{\prime}$ of order $p$ in $Y^{\prime}$, including the fact that an appropriate analogue of $\mathfrak{S}\left(Y^{\prime}, i^{\prime}\right)$ is nonempty.

Theorem 4. Let $q$ be a power of 2, and assume $Y=O^{2^{\prime}}(Y)$ is an almost simple group of Lie type over $\mathbb{F}_{q}$, or $Y={ }^{2} F_{4}(2)^{\prime}$ is the Tits group. Let $i$ be an involution in $Y$. Then one of the following holds:

(1) $\subseteq(Y, i)$ is nonempty.

(2) $Y \cong \operatorname{Sp}_{n}(q)$ and $i$ is of type $c_{l}$ for some even integer $l>2$.

(3) $Y \cong \Omega_{n}^{\varepsilon}(q), n \geq 8$, and $i$ is of type $c_{l}$ for some even $l>2$.

(4) $Y \cong E_{7}(q)$ and $i$ is of type $u$ or $v$.

(5) $Y \cong E_{8}(q)$ and $i$ is of type $v$.

The exclusions in (2)-(5) of Theorem 4 may not be necessary. We do not need to consider such involutions in proving Theorem 2 , and did not immediately see how to show $\mathbb{S}(Y, i) \neq \emptyset$ for such involutions (except in case (3)), so we leave the question open. However there do seem to be some serious difficulties involved with case (2).

To conclude the introduction we point out that Theorem 3 is proved in $\S 8$, the proof of Theorem 4 is completed in $\S 15$, and the proof of Theorem 2 is completed in $\$ 16$. Our basic references are [A4] for notation and terminology involving finite groups and [GLS3] for notation, terminology, and information about the finite simple groups. 


\section{Sidki pairs}

In this section $G$ is a finite group and $V$ is a nontrivial elementary abelian 2-subgroup of $G$.

Given a $V$-invariant subgroup $H$ of $G$, define $\mathscr{D}(H, V)$ to be the set of $(h, v) \in$ $H \times V$ such that $h$ is of odd order and $v$ inverts $h$. Let $\mathcal{B}(H, V)$ consist of those $(h, v) \in$ $\mathscr{D}(H, V)$ such that $C_{V}(h)=1$. Recall from the Introduction that the pair $(G, V)$ is a Sidki pair if $\mathcal{B}(G, V)$ is empty. Write $\mathcal{P}$ for the set of Sidki pairs. A minimal counter example to Theorem 2 is a Sidki pair $(G, V)$ such that $O_{2}(G) \cap V=1,|G|$ is minimal subject to this constraint, and $|V|$ is minimal subject to both constraints. Write $\mathcal{Q}$ for the set of minimal counter examples to Theorem 2 .

Lemma 1.1. Assume $(G, V) \in \mathcal{P}$. Then:

(1) If $V \leq H \leq G$, then $(H, V) \in \mathcal{P}$.

(2) Suppose $K \unlhd G$ with $V \cap K=1$, and set $G^{*}=G / K$. Then $\left(G^{*}, V^{*}\right) \in \mathcal{P}$.

Proof. As $\mathscr{B}(H, V) \subseteq \mathscr{B}(G, V)$, (1) holds. Let $x \in G^{*}$ be an element of odd order and let $y \in V^{*}$ be an involution that inverts $x$. Then $D:=\langle x, y\rangle$ is a dihedral group of order $2 m$, where $m$ is odd, and there exists $v \in V$ with $v^{*}=y$. Thus there exists $g \in G$ such that $D=\left\langle v^{*},\left(v^{g}\right)^{*}\right\rangle$. Let $E=\left\langle v, v^{g}\right\rangle$. Then there exists $h \in E$ of odd order such that $h^{*}=x$. Since $v$ inverts $h$ and $(G, V) \in \mathcal{P}, C_{V}(h) \neq 1$. As $V \cap K=1, C_{V}(h) \cong C_{V}(h)^{*} \leq C_{V^{*}}\left(h^{*}\right)=C_{V^{*}}(x)$, so $C_{V^{*}}(x) \neq 1$.

Lemma 1.2. If $(G, V) \in \mathcal{P}$ and $|V|=2$, then $V \leq O_{2}(G)$.

Proof. Assume otherwise. Then by the Baer-Suzuki Theorem (cf. 39.6 in [A4]), a generator $v$ of $V$ inverts a nontrivial element $x$ of odd order, and as $|V|=2$, $C_{V}(x)=1$, contradicting $(G, V) \in \mathcal{P}$.

The following lemma, and Lemma 1.5 below, should be compared with Theorem 2.1 in $[\mathrm{Si}]$.

Lemma 1.3. Assume $(G, V) \in \mathcal{P}$ and $E$ is of index 2 in $V$ with $(G, E) \notin \mathcal{P}$. Then $V \leq O_{2}\left(C_{G}(E)\right)$.

Proof. Assume otherwise. Then $|V|>2$ by 1.2. By hypothesis there is $(x, e) \in$ $\mathscr{B}(G, E)$, and as $(G, V) \in \mathcal{P}$, there is $1 \neq v \in C_{V}(x)$. As $(x, e) \in \mathscr{B}(G, E)$, $C_{E}(x)=1$ so $v \in V-E$. Hence by assumption, $v \notin O_{2}\left(C_{G}(E)\right)$, so by the Baer-Suzuki Theorem, there is $g \in C_{G}(E)$ such that $y:=v v^{g}$ is nontrivial of odd order. Then $\left(G, V^{g}\right) \in \mathcal{P}$ and $(x, e) \in \mathscr{D}\left(G, V^{g}\right)$, so there exists $1 \neq u \in C_{V^{g}}(x)$. As $C_{E}(x)=1, u=v^{g} a$ for some $a \in E$. Then $y a=v v^{g} a=v u$ centralizes $x$, and $y$ centralizes $a$, so also $(y a)^{2}=y^{2}$ centralizes $x$. Then as $|y|$ is odd, $[x, y]=1$. 
Therefore $|x y|$ is odd and $e v$ inverts $x y$, so there is $1 \neq w \in C_{V}(x y)$. If $w \in E$ then $x y=(x y)^{w}=x^{w} y$, so $w \in C_{E}(x)=1$, a contradiction. Thus $w=b v$ for some $b \in E$. Therefore $x y=(x y)^{w}=x^{b} y^{-1}$, so $x^{b}=x y^{2}$. Then as $[b, y]=1$,

$$
x=x^{b^{2}}=\left(x y^{2}\right)^{b}=x^{b} y^{2}=x y^{4},
$$

contradicting $|y|$ odd.

Lemma 1.4. If $(G, V) \in \mathcal{P}$ and $|V| \leq 4$, then $V \cap O_{2}(G) \neq 1$.

Proof. Assume otherwise and choose $v \in V^{\#}$. By Baer-Suzuki, $v$ inverts a nontrivial element $x$ of odd order in $G$. As $(G, V) \in \mathcal{P}$, there is $1 \neq e \in C_{V}(x)$. But by 1.2 and $1.3, V \leq O_{2}\left(C_{G}(e)\right)$, a contradiction.

Lemma 1.5. Assume that $(G, V) \in \mathcal{P}$, but for each maximal subgroup $E$ of $V$, $(G, E) \notin \mathcal{P}$. Then $V$ centralizes each $V$-invariant subgroup of $G$ of odd order.

Proof. Let $X$ be a $V$-invariant subgroup of $G$ of odd order, and $\mathscr{E}$ the set of maximal subgroups of $V$. Then (cf. Exercise 8.1 in [A4]) $X=\left\langle C_{X}(E): E \in \mathscr{E}\right\rangle$. Furthermore, for $E \in \mathcal{E}$ we have $V \leq O_{2}\left(C_{G}(E)\right)$ by hypothesis and 1.3, so $\left[C_{X}(E), V\right] \leq O_{2}\left(C_{G}(E)\right) \cap X=1$, and hence $V$ centralizes $X$.

Lemma 1.6. Let $U \leq V$ be a nontrivial subgroup and let $H$ be a $U$-invariant subgroup of $G$. Then $(H U, U) \in \mathcal{P}$ iff $\mathcal{B}(H, U)=\emptyset$.

Proof. As the 2-group $U$ acts on $H, O^{2}(H U)=O^{2}(H)$, so $B(H, U)=\mathscr{B}(H U, U)$.

Lemma 1.7. Assume $(G, V) \in \mathcal{P}$, let $v \in V$ and let $x \in G$ be an element of odd order inverted by $v$. Set $D:=\langle v, x\rangle$ and $C:=C_{G}(D)$. Then $(C, C \cap V) \in \mathcal{P}$.

Proof. Let $W:=C \cap V$, and notice that since $(G, V) \in \mathcal{P}, W \neq 1$. Pick $1 \neq w \in W$ and let $y \in C$ be an element of odd order inverted by $w$. Then $v w$ inverts $x y$, so there exists $u \in V^{\#}$ such that $u \in C_{V}(x y)$. Since $w, x y \in C_{G}(u)$ also $(x y)(x y)^{w}=$ $x y x y^{-1}=x^{2} \in C_{G}(u)$. But $|x|$ is odd, so $u \in C$ and hence $u \in W$. Note that $u$ centralizes $y$, so we see that $(C, W) \in \mathcal{P}$.

Our goal in the remainder of the section is to prove that if $(G, V)$ is a minimal counter example to Theorem 2 then $G$ is almost simple, and to obtain further results on a minimal counter example. Thus in the remainder of the section we assume:

Hypothesis 1.8. $(G, V)$ is a minimal counter example to Theorem 2 . 
Lemma 1.9. Let $1 \neq U \leq V$ and assume that $H$ is a nontrivial $U$-invariant subgroup of $G$ such that $H U \neq G$.

(1) If $C_{U}(H)=1$ and $O_{2}(H)=1$, then $(H U, U) \notin \mathcal{P}$.

(2) If $U=V$ and $O_{2}(H)=1$, then $C_{V}(H) \neq 1$.

Proof. Notice that (2) is an immediate corollary of (1) because by Lemma 1.1(1), $(H V, V) \in \mathcal{P}$.

Assume the hypothesis of (1) with $(H U, U) \in \mathcal{P}$. Then by minimality of $G$ in Hypothesis 1.8, $U \cap O_{2}(H U) \neq 1$, and so $\left[U \cap O_{2}(H U), H\right] \leq O_{2}(H U) \cap H=1$, contradicting $C_{U}(H)=1$.

Lemma 1.10. (1) If $V \leq H<G$, then $V \cap O_{2}(H) \neq 1$.

(2) If $H$ is a proper subgroup of $G$ of odd index, then $1 \neq V \cap O_{2}(K)$ for some $K \in H^{G}$.

Proof. Under the hypothesis of (1), $(H, V) \in \mathcal{P}$ by 1.1(1), so (1) follows from minimality of $G$. Then (2) follows from (1) and Sylow's Theorem.

Lemma 1.11. $V$ centralizes each $V$-invariant subgroup of $G$ of odd order.

Proof. This follows from the minimality of $V$ in Hypothesis 1.8, and from 1.5.

Lemma 1.12. Let $X_{i} \leq G$ be $V$-invariant subgroups of $G$ for $i=1,2$, such that $X:=\left\langle X_{1}, X_{2}\right\rangle=X_{1} \times X_{2}, O_{2}(X)=1$ and $X_{i} V \neq G$, then $C_{V}(X) \neq 1$.

Proof. Assume that $C_{V}(X)=1$ and set

$$
V_{1}:=C_{V}\left(X_{2}\right) .
$$

By Lemma 1.9(2) $V_{1} \neq 1$. Since $C_{V_{1}}\left(X_{1}\right)=1$, Lemma 1.9(1) implies that $\left(X_{1} V_{1}, V_{1}\right) \notin \mathcal{P}$, so by Lemma 1.6 we may pick $\left(h_{1}, v_{1}\right) \in \mathscr{B}\left(X_{1}, V_{1}\right)$. Set

$$
V_{2}:=C_{V}\left(h_{1}\right) .
$$

Then $V_{2} \cap V_{1}=1$ and hence $C_{V_{2}}\left(X_{2}\right)=V_{2} \cap C_{V}\left(X_{2}\right)=V_{2} \cap V_{1}=1$. Hence, again, we may choose $\left(h_{2}, v_{2}\right) \in \mathscr{B}\left(X_{2}, V_{2}\right)$. Note that

$$
C_{V}\left(h_{1} h_{2}\right)=C_{V}\left(h_{1}\right) \cap C_{V}\left(h_{2}\right)=V_{2} \cap C_{V}\left(h_{2}\right)=C_{V_{2}}\left(h_{2}\right)=1 .
$$

However as $X=X_{1} \times X_{2}$ with $h_{i} \in X_{i},\left[h_{1}, h_{2}\right]=1$ so $h_{1} h_{2}$ is inverted by $v_{1} v_{2}$, contrary to our hypothesis that $(G, V) \in \mathcal{P}$.

Lemma 1.13. $F(G)=1$. 
Proof. Assume that $O_{2}(G) \neq 1$. As $(G, V) \in \mathcal{Q}, V \cap O_{2}(G)=1$. Then we conclude from Lemma 1.1 (2) and minimality of $G$ that $O_{2}\left(G / O_{2}(G)\right) \neq 1$, which is absurd.

Thus $O_{2}(G)=1$. Assume that $K:=F(G) \neq 1$, and set $G^{*}:=G / K$. As $O_{2}(G)=1, K$ is of odd order, so $V \cap K=1$. Thus $\left(G^{*}, V^{*}\right) \in \mathcal{P}$ by Lemma 1.1 (2). Then by minimality of $G, 1 \neq V^{*} \cap O_{2}\left(G^{*}\right)$. Let $U$ be the preimage in $V$ of $V^{*} \cap O_{2}\left(G^{*}\right)$. As $E(G)^{*} \leq E\left(G^{*}\right),[U, E(G)] \leq K$, so $U$ centralizes $E(G)$ (cf. 31.6 .3 in [A4]). Further $V$ centralizes $K$ by 1.11, so $U$ centralizes $F^{*}(G)$, contradicting $\mathrm{O}_{2}(G)=1$.

We can now prove

Proposition 1.14. Assume that $(G, V)$ is a minimal counter example to Theorem 2. Then $G$ is almost simple and $G=V F^{*}(G)$.

Proof. We first claim that

(1) $V$ acts transitively via conjugation on the components of $G$.

Let $\Omega$ be the set of components of $G$ and let $\Omega_{1}$ be an orbit of $V$ on $\Omega$. Assume that $\Omega_{2}=\Omega \backslash \Omega_{1}$ is nonempty. Set $X_{i}=\left\langle\Omega_{i}\right\rangle, i=1,2$ and note that we get a contradiction to Lemma 1.12. This establishes (1).

Let $L \in \Omega$. We next claim that

(2) $V_{1}:=N_{V}(L) \neq 1$.

Assume (2) fails and let $q$ be an odd prime dividing $|L|$ and $Q_{1} \in \operatorname{Syl}_{q}(L)$. Then $Q:=\left\langle Q_{1}^{V}\right\rangle$ is a $V$-invariant Sylow $q$-subgroup of $F^{*}(G)$, and $V$ is faithful on $Q$, contrary to 1.11. This establishes (2).

We next show

(3) $\left(L V_{1}, V_{1}\right) \in \mathcal{P}$.

First, for $1 \neq x \in L, C_{G}(x) \leq N_{G}(L)$. Thus if $x$ has odd order and is inverted by some $v \in V_{1}$, then $C_{V}(x) \neq 1$, and since $C_{V}(x) \leq N_{G}(L), C_{V}(x) \leq V_{1}$. So, by $1.6,(3)$ is established.

If $G=L V$ then the proposition holds, so we may assume otherwise. Then by (3) and the minimality of $G, C_{V_{1}}(L) \neq 1$. However as $V$ is abelian, $C_{V_{1}}(L) \leq$ $C_{V_{1}}\left(F^{*}(G)\right)=1$ by (1), a contradiction.

Lemma 1.15. Assume $u$ is an involution in $G$ and $x$ is a nontrivial element of odd order inverted by $u$. Set $D:=\langle u, x\rangle$ and suppose that either

(1) $O_{2}\left(C_{G}(D)\right)=1$, or

(2) $\mathrm{O}_{2}\left(C_{G}(D)\right)$ contains a unique involution $t$, and either $t \notin V$ or $\left\langle t^{C_{G}(u)}\right\rangle$ is not elementary abelian.

Then $u \notin V$. 
Proof. Suppose $u \in V$ and let $H=C_{G}(u), C=C_{G}(D)$, and $h \in H$. Set $W_{h}=C_{V^{h}}(D)$. By $1.7,\left(C, W_{h}\right) \in \mathcal{P}$, so by the minimality of $G, W_{h} \cap O_{2}(C) \neq 1$ and hence $t \in W_{h}$. We have

$$
t \in \bigcap_{h \in H} W_{h} \leq \bigcap_{h \in H} V^{h}=: V_{H},
$$

so $t \in V$ and as $V_{H}$ is an elementary abelian normal subgroup of $H,\left\langle t^{H}\right\rangle$ is elementary abelian, a contradiction.

\section{Alternating groups}

In this section we prove:

Theorem 2.1. Assume $(G, V)$ is a minimal counter example to Theorem 2. Then $G$ is not an alternating or symmetric group.

Let $\Omega=\{1, \ldots, n\}, S$ the symmetric group on $\Omega$, and $A$ the alternating group on $\Omega$. Assume $(G, V)$ is a minimal counter example to Theorem 2 and $G=S$ or $A$. For $X \subseteq S$, write $M(X)$ for the set of points of $\Omega$ moved by $X$.

Lemma 2.2. (1) $n \geq 5$.

(2) $V$ acts on no partition $\Gamma=\left\{\Omega_{1}, \Omega_{2}\right\}$ of $\Omega$ with $\left|\Omega_{i}\right|>4$ for $i=1,2$.

Proof. Part (1) follows from Proposition 1.14, which says that $G$ is almost simple. Suppose $V$ acts on $\Gamma$ as in (2), and let $H=N_{G}(\Gamma)$. Then $V \leq H<G$ with $\mathrm{O}_{2}(H)=1$, contrary to 1.10 .

Lemma 2.3. (1) $M(v)=\Omega$ for each $v \in V^{\#}$.

(2) Each orbit of $V$ on $\Omega$ is regular.

(3) $|V| \geq 8$.

(4) $n \geq 8$.

Proof. Assume (1) fails and pick $v \in V^{\#}$ so that $M(v) \neq \Omega$, and $m=|M(v)|$ is maximal subject to this constraint. Then $v$ inverts a cycle $h \in G$ of length $m+1$. Now $(h, v) \in \mathscr{D}(G, V)$, so as $\mathscr{B}(G, V)=\emptyset$, there exists $1 \neq u \in C_{V}(h)$. Then $M(h) \subseteq \operatorname{Fix}(u)$, so $M(u v)=M(u) \cup M(v)$ is of order $m+|M(u)|>m$. Further $u v$ fixes the fixed point of $v$ on $M(h)$, so $M(u v) \neq \Omega$, contrary to the maximality of $m$. Thus (1) is established.

Part (1) implies (2), while (3) follows from 1.4. By (2) and (3), $n \equiv 0 \bmod 8$, so (4) follows. 
Lemma 2.4. $V$ is regular on $\Omega$.

Proof. Let $m=|V|$. By 2.3 (2), $V$ has $r=n / m$ regular orbits of length $m$ on $\Omega$, and we may assume $r>1$. By 2.3 (3), $m \geq 8$. Let $\Delta$ be an orbit of $V$ on $\Omega$, and set $\Sigma=\Omega-\Delta$ and $H=G_{\Delta}$. Thus $H$ is the alternating or symmetric group on $\Sigma$, and as $|\Sigma| \geq m \geq 8, O_{2}(H)=1$. Hence $C_{V}(H) \neq 1$ by $1.9(2)$. But $C_{V}(H)$ fixes $\Sigma$ pointwise, contrary to $2.3(1)$.

Lemma 2.5. $n=8$.

Proof. Let $U$ be a subgroup of index 2 in $V$ and $\Gamma$ the set of orbits of $U$ on $\Omega$. As $V$ is regular on $\Omega, U$ has two orbits of length $n / 2$ and $V$ acts on $\Gamma$. Now the lemma follows from 2.2 (2) and 2.3 (4).

We are now in a position to obtain a contradiction, establishing Theorem 2.1. By 2.4 and $2.5, G \cong L_{4}(2)$ and we may choose $V$ to be the group of transvections with a fixed axis $P$ on the natural module $M$ for $G$. Let $v \in V^{\#}$ and pick $h$ of order 3 in $G$ inverted by $v$. Then $\operatorname{dim}([M, h])=2$ and $M=[M, h]+P$. However $C_{V}(h)$ centralizes $[M, h]$ and $P$, so $C_{V}(h)$ centralizes $M=[M, h]+P$ and hence $C_{V}(h)=1$. Thus $(h, v) \in \mathscr{B}(G, V)$, for our contradiction.

\section{Some strongly real elements in sporadic groups}

In this section $L$ is a sporadic group. Our notation for conjugacy classes in $L$ come from [GLS3], although we sometimes write ' $r$ ' rather than ' $r A$ ' if $L$ has a unique class of elements of order $r$. The information about the normalizers of elements of prime order in $L$ comes from [GLS3] as well; sometimes the appeals to this information are implicit rather than explicit.

For a group $H$ we write $\operatorname{Inv}(H)$ for the set of involutions of $H$ and for $x \in H$, $N_{H}(x):=N_{H}(\langle x\rangle)$. Given $L$-invariant subsets $A, B$ of $G$, we sometimes write $[A, B]=1$ to indicate that $[a, b]=1$ for some $a \in A$ and $b \in B$. We write $t \rightsquigarrow x$ to indicate that the involution $t$ inverts $x$, and write $A \rightsquigarrow B$ to indicate that $a \rightsquigarrow b$ for some $a \in A$ and $b \in B$.

Lemma 3.1. (1) Let $M \cong M_{24}$ and let $\Omega$ be a set of 24 points permuted transitively by $M$. Then $M$ has two classes of involutions $2 A$ and $2 B$ such that:

(a) For $a \in 2 A, C_{M}(a) \cong L_{3}(2) / D_{8}^{3}$ and $\operatorname{Fix}(a)$ is an octad in $\Omega$.

(b) For $b \in 2 B, C_{M}(b) \cong S_{5} / E_{64}$ and $b$ has no fixed point on $\Omega$.

(c) $2 B \rightsquigarrow 11 A$.

(2) Let $L \cong M_{22}$, then $2 C \rightsquigarrow 11 A$. 
Proof. (1): Parts (a) and (b) appear in [A8, 21.1]. Let $X \leq M$ be of order 11. As the stabilizer $L_{3}(4)$ in $M$ of 3 points of $\Omega$ is an $11^{\prime}$-group, $|\operatorname{Fix}(X)|=2$ and $X$ has two orbits $\Omega_{i}, i=1,2$, of length 11 on $\Omega$. An involution $t \in M$ that inverts $X$ acts on Fix $(X)$ and either fixes one point in $\Omega_{i}$ for $i=1$ and 2, or interchanges $\Omega_{1}$ and $\Omega_{2}$. Thus $\mid$ Fix $(t) \mid \leq 4$, so $t \in 2 B$.

(2): View $\operatorname{Aut}(L)$ as the global stabilizer in $M=M_{24}$ of two points. Let $c \in$ $\operatorname{Inv}(\operatorname{Aut}(L)) \backslash L$ with $c \rightsquigarrow 11$. By (1), $c$ is in the $M$-class $2 B$. Thus $7 \notin \pi\left(C_{M}(c)\right)$, so $c$ is of type $2 C$ in $\operatorname{Aut}(L)$.

Lemma 3.2. The following table lists groups $L$ and classes $\mathcal{O}$ and $\mathcal{A}$ in $\operatorname{Aut}(L)$ such that

(i) $|\operatorname{Out}(L)|=2$ and $\mathcal{A}$ is a class of outer involutions in $\operatorname{Aut}(L)$;

(ii) for $x \in \mathcal{O},\left|C_{\mathrm{Aut}(L)}(x)\right|$ is odd and $\mathcal{A} \rightsquigarrow \mathcal{O}$.

\begin{tabular}{|l|l|l|l|l|}
\hline$L$ & $M_{12}$ & $M_{22}$ & $J_{3}$ & $O^{\prime} N$ \\
\hline $\mathcal{O}$ & $11 A$ & $11 A$ & $19 A$ & $31 A$ \\
\hline $\mathcal{A}$ & $2 C$ & $2 C$ & $2 B$ & $2 B$ \\
\hline
\end{tabular}

Proof. Observe $p=|x|$ is an odd prime, and from [GLS3], $\langle x\rangle \in \operatorname{Syl}_{p}(L),\left|C_{L}(x)\right|$ is odd, $|\operatorname{Out}(L)|=2$, and for each involution $a \in \operatorname{Aut}(L) \backslash L, p \notin \pi\left(C_{L}(a)\right)$. Thus, by a Frattini argument, some involution $t \in \operatorname{Aut}(L) \backslash L$ acts on $\langle x\rangle$, and then, as $p \notin \pi\left(C_{L}(t)\right), t$ inverts $x$.

Next, if $L \neq M_{22}$, then there is a unique class $\mathcal{A}$ of outer involutions in $\operatorname{Aut}(L)$ so $t \in \mathcal{A}$ and the lemma holds in this case. If $L=M_{22}$ then the lemma follows from Lemma $3.1(2)$.

Lemma 3.3. The following table lists classes $\mathcal{O}$ and $\mathcal{A}$ in $L$ such that for $x \in \mathcal{O}$, $\left|C_{L}(x)\right|$ is odd and $\mathcal{A} \rightsquigarrow \mathcal{O}$.

\begin{tabular}{|l|l|l|l|l|l|l|l|}
\hline$L$ & $J_{2}$ & $J_{3}$ & $J_{4}$ & $H S$ & $M c L$ & $R u$ & $O^{\prime} N$ \\
\hline $\mathcal{O}$ & $7 A$ & $17 A$ & $23 A$ & $7 A$ & $5 B$ & $29 A$ & $11 A$ \\
\hline $\mathcal{A}$ & $2 B$ & $2 A$ & $2 B$ & $2 B$ & $2 A$ & $2 B$ & $2 A$ \\
\hline
\end{tabular}

Proof. The $J_{3}, M c L$ and $O$ ' $N$ entries of the table follow from the fact that these groups have a unique class of involutions and from the structure of the normalizers of subgroups of prime order in these groups.

$J_{2}$ : Let $L \cong J_{2}$ and let $c$ be an outer involution in $\operatorname{Aut}(L)$. Then $K=C_{L}(c) \cong$ $\mathrm{PGL}_{2}(7)$ has Sylow 2-subgroup $S \cong D_{16}$ and two classes $z^{K}$ and $t^{K}$ of involutions, with $z$ 2-central in $K$. As $Z(S)$ contains a 2-central involution of $G, z$ is 2-central in $G$, and hence of type $2 A$. Then as non-2-central involutions of $L$ of type $2 B$ also centralize outer involutions (cf. [GLS3, pg. 268]), $t \in 2 B$. Finally $t \rightsquigarrow 7$ in $K$. 
$J_{4}: L \cong J_{4}$ has two classes of elements of order 11 and two classes of involutions. Further, for each $t \in \operatorname{Inv}(L)$, Sylow 11-subgroups of $C_{L}(t)$ have order 11, so $C_{L}(t)$ is transitive on its subgroups of order 11. Since each element of order 11 in $L$ is centralized by an involution, we conclude that:

(1) For $X \in\{A, B\}$, there exists a unique class $11_{X}$ of elements of order 11 such that $\left[11_{X}, 2 X\right]=1$. Further $11_{A} \neq 11_{B}$.

Next let $Y$ be generated by $y \in 11 A$. By [GLS3], $N_{L}(Y) \cong\left(\mathrm{GL}_{2}(3) \times \mathbb{Z}_{5}\right) / 11^{1+2}$ is strongly 11 -embedded in $L$. Let $Q=O_{11}\left(N_{L}(Y)\right)$. Then there is an involution $z \in C_{L}(Y)$ inverting $Q / Y$, and $3 \in \pi\left(C_{L}(y, z)\right)$, so $z \in 2 A$, since if $z \in 2 B$ then $3 \notin \pi\left(C_{C_{L}(z)}(y)\right)$. It follows from (1) that:

(2) $11_{X}=11 X$ for $X \in\{A, B\}$.

Further as $N_{L}(Y)$ is strongly 11 -embedded in $L$, each $b \in Q-Y$ is in $11 B$, and $z$ inverts some such elements, so:

(3) $2 A \rightsquigarrow 11 B$.

Let $b \in 11 B$ and $v \in C_{L}(b) \cap 2 B$. Then $C_{L}(v)$ is contained in a maximal subgroup $M \cong M_{24} / E_{2^{11}}$, so it follows from the structure of $M_{24}$ that $b \in N_{M}(X)$ for some $X \leq M$ of order 23. Finally $N_{L}(X)$ is Frobenius of order 23 22 , so (2) implies that $2 B \rightsquigarrow 23$.

$H S$ : Let $L \cong H S$ and let $d \in 2 D$. Then $K:=C_{L}(d) \cong S_{8}$. Consider the covering group $\hat{L}$ of $L$, and let $\hat{K}$ be the preimage of $K$ in $\hat{L}$. By [GLS3], $Z:=Z(\hat{L})$ is of order 2 , and the involutions in $K \backslash E(K)$ lift in $\hat{L}$ to elements of order 4 . As involutions of type $2 A, 2 B$ in $L$ lift to involutions, elements of order 4 in $\hat{L}$, respectively, it follows that involutions in $K \backslash E(K)$ are of type $2 B$. Since $L$ contains a unique class of elements of order 7, and an element of order 7 in $K$ is inverted by an involution of cycle type $2^{3}$, we are done.

$R u$ : There are two classes of involutions in $R u$ and only $[2 B, 7]=1$. Next for $x \in 29, N_{L}(x)$ is Frobenius of order 29.14, and hence $\operatorname{Inv}\left(N_{L}(x)\right) \subset 2 B$.

Lemma 3.4. If $L \cong M_{12}$, then $2 A \rightsquigarrow 5 A, 2 B \rightsquigarrow 5 A$, and for $x \in 5 A, \operatorname{Inv}\left(C_{L}(x)\right) \subset$ $2 A$.

Proof. First, $L$ has two classes of involutions, $2 A$ and $2 B$, and one class $5 A$ of elements of order 5. If $t \in 2 A$, then $t$ has no fixed points on the set $\Omega$ of 12 points permuted by $L$, while $s \in 2 B$ fixes 4 points. Further $L$ contains a transitive subgroup $K \cong L_{2}(11)$ and for $\omega \in \Omega, K_{\omega}$ is a Borel subgroup, so the involutions in $K$ are in the class $2 A$ and inside $K, 2 A \rightsquigarrow 5 A$. Next, $2 B \rightsquigarrow 5$, inside $L_{\omega} \cong M_{11}$. Finally, since $5 \notin \pi\left(C_{L}(2 B)\right), \operatorname{Inv}\left(C_{L}(x)\right) \subset 2 A$.

Lemma 3.5. If $L \cong J_{2}$, then $2 A \rightsquigarrow 5 A$ and for $y \in 5 A$, $\operatorname{Inv}\left(C_{L}(y)\right) \subset 2 B$.

Proof. By [GLS3], [2B, 5A] $=1$, so since $C_{L}(y) \cong \mathbb{Z}_{5} \times A_{5}, \operatorname{Inv}\left(C_{L}(y)\right) \subset 2 B$. Let $b \in \operatorname{Inv}\left(C_{L}(y)\right)$. By [GLS3], $C_{L}(b) \cong E_{4} \times A_{5}$, and $\operatorname{Inv}\left(C_{L}(b)\right) \cap 2 A=$ $\operatorname{Inv}\left(E\left(C_{L}(b)\right)\right)$. Thus inside $C_{L}(b), 2 A \rightsquigarrow 5 A$. 
Lemma 3.6. Assume $L \cong \mathrm{Co}_{2}$, let $x \in 5 B$, and set $N:=N_{L}(x)$. Then:

(1) $N \cong F_{20} \times S_{5}$, where $F_{20}$ is the Frobenius group of order 20 .

(2) If $s \in \operatorname{Inv}(N)$ and $C_{L}(s, x) \cong S_{5}$, then $s \in 2 B$.

(3) $2 C \rightsquigarrow 7 A$ and for $z \in 7 A, \operatorname{Inv}\left(C_{L}(z)\right) \subset 2 A \cup 2 B$.

Proof. Part (1) appears in [GLS3]. Let $t$ be a transposition in $C_{L}(x)$. We claim that $t \in 2 A$. Set $C:=C_{L}(t)$ and $C^{*}:=C / O_{2}(C)$. Then $x^{*}$ is of order 5 in $C^{*}$ such that $C_{C^{*}}\left(x^{*}\right) \cong \mathbb{Z}_{5} \times S_{3}$. Now the claim follows from the structure of centralizers of involutions in $L$. By the claim:

(i) $[5 B, 2 A]=1$.

Next from Section 24 in [A8], there exists a subgroup $H S \cong K \leq L$. Note that $K$ contains a Sylow 5-subgroup of $L$, and then from the structure of the centralizers of elements of order 5 in $L$ and $K$ :

(ii) For $X \in\{5 A, 5 B\}$, the $K$-class $X$ is contained in the $L$-class $X$.

Similarly for $u$ in the $K$-class $2 B, C_{K}(u)$ contains an $\operatorname{Aut}\left(A_{6}\right)$-section, so:

(iii) The $K$-class $2 B$ is contained in the $L$-class $2 C$.

Next from [GLS3], there exists $S_{8} \cong S \leq K$. Let $x_{S}$ be an element of order 5 in $S$. Then $3 \in \pi\left(C_{K}\left(x_{S}\right)\right)$, so $x_{S}$ is in the $K$-class $5 B$. Further there exists $t \in \operatorname{Inv}(S)$ centralizing $x$ with $C_{S}(t) \cong \mathbb{Z}_{2} \times S_{6}$, so $t$ is in the $K$-class $2 B$. Hence from (ii) and (iii):

(iv) $[5 B, 2 C]=1$.

Now for each $t \in \operatorname{Inv}(L)$, Sylow 5 -subgroups of $C_{L}(t)$ have order 5 , so $C_{L}(t)$ is transitive on its subgroups of order 5. Hence by (i) and (iv), for $y \in 5 A, C_{L}(y) \cap 2 A=$ $\emptyset=C_{L}(y) \cap 2 C$, so:

(v) For $y \in 5 A, \operatorname{Inv}\left(C_{L}(y)\right) \subseteq 2 B$.

If $i$ is in the $K$-class $2 B$, then $C_{K}(i) \cong \mathbb{Z}_{2} \times \operatorname{Aut}\left(A_{6}\right)$, so:

(vi) If $t \in \operatorname{Inv}(K)$ is a square in $K$, then $t$ is in the $K$-class $2 A$.

Next an element $y$ in the $K$-class $5 A$ is centralized in $K$ by an involution $i$ which is a square in $C_{K}(y)$, so $i$ is in the $K$-class $2 A$ by (vi). By (ii), $y$ is in the $L$-class $5 A$, so by (v), $i$ is in the $L$-class $2 B$. Thus we have shown:

(vii) The $K$-class $2 A$ is contained in the $L$-class $2 B$.

By (ii), we may assume that $x \in K$ and $N_{K}(x) \cong F_{20} \times A_{5}$. Let $s$ be an involution in $N_{K}(x)$ with $C_{K}(s, x) \cong A_{5}$. By (vi) and (vii), $s$ is in the $L$-class $2 B$, so (2) follows.

It remains to prove (3). We may assume that $z \in K$. By 3.3, $z$ is inverted by an involution $j$ in the $K$-class $2 B$, so $j$ is in the $L$-class $2 C$ by (iii). Since for $c \in 2 C$, $7 \notin \pi\left(C_{L}(c)\right),(3)$ follows.

Lemma 3.7. Let $L \cong H e$. Then:

(1) $2 B \rightsquigarrow 17$ and $\left|C_{\mathrm{Aut}(L)}(x)\right|=17$, for $x \in 17$.

(2) Let $x \in 5 A$. Then $x$ is inverted by some element a in $2 A$ with $C_{L}(a, x) \cong A_{5}$ and $C_{\mathrm{Aut}(L)}(a, x) \cong S_{5}$. 
Proof. Let $a \in 2 A$. Then $C_{L}(a)$ has a subgroup $E_{4} \times L_{3}(4)$ of index 2 , so if $u \in L$ with $|u|=8$, then $u^{4} \in 2 B$. Let $y \in 17$. Then $N_{L}(y)$ is Frobenius of order $17 \cdot 8$, so the first part of (1) follows, and the second is a consequence of [GLS3]. Part (2) follows from 42.14 in [A8].

\section{Sporadic groups in Theorem 2}

In this section we begin to prove the following result.

Theorem 4.1. If $(G, V)$ is a minimal counter example to Theorem 2 , then $F^{*}(G)$ is not sporadic.

Assume that $(G, V)$ is a minimal counter example to Theorem 2 with $L=F^{*}(G)$ sporadic. By Proposition 1.14, $G=V L$. We begin with a series of reductions.

Lemma 4.2. Assume $a$ is an involution in $G$ and $x$ is an element of odd order inverted by $a$. Set $D=\langle a, x\rangle$. Then each of the following imply that $a \notin V$ :

(1) $O_{2}\left(C_{G}(D)\right)=1$.

(2) $v^{G} \cap C_{G}(D)=\emptyset$ for each $v \in V^{\#}$.

Proof. Part (1) is sufficient by 1.15 (1). Further if $a \in V$, then $(x, a) \in \mathscr{D}(G, V)$, so as $(G, V) \in \mathcal{P}, C_{V}(D) \neq 1$; thus (2) is sufficient.

Lemma 4.3. Assume a is an involution in $G$ and $x$ is an element of odd order inverted by a. Set $D=\langle a, x\rangle$ and assume $O_{2}\left(C_{G}(D)\right)$ has a unique involution $t$. Then each of the following imply that $a \notin V$ :

(1) $\mathrm{O}_{2}\left(C_{G}(a)\right)=\langle a\rangle$.

(2) $\left\langle t^{C_{G}(a)}\right\rangle$ is not elementary abelian.

Proof. Part (2) is sufficient by 1.15 (2). Then (1) is sufficient by (2).

Lemma 4.4. $V \cap L \neq 1$.

Proof. This follows as $|\operatorname{Out}(L)| \leq 2$ and $|V| \geq 8$ by 1.4.

Lemma 4.5. $L$ is not $M_{11}, M_{23}, J_{1}$, Ly or $F_{3}$.

Proof. For each of the groups, $\operatorname{Out}(L)=1$, and $L$ has one class $a^{L}$ of involutions. We indicate a class $\mathcal{O}=x^{L}$ of elements of odd prime order in $L$ satisfying the hypothesis of Lemma 4.2(1), and appeal to that lemma.

\begin{tabular}{|l|l|l|l|l|l|}
\hline$L$ & $M_{11}$ & $M_{23}$ & $J_{1}$ & $L y$ & $F_{3}$ \\
\hline $\mathcal{O}$ & $5 A$ & $5 A$ & $7 A$ & $31 A$ & $19 A$ \\
\hline
\end{tabular}


Lemma 4.6. If $L$ is $M_{12}, J_{3}$, or $O{ }^{\prime} N$, then $G=L$.

Proof. Let $\mathcal{A}=a^{L}$ and $\mathcal{O}=x^{L}$ be as in Lemma 3.2. Then, by Lemma 3.2 and Lemma 4.2(1), $V \cap \mathcal{A}=\emptyset$. But $\mathcal{A}$ is the unique class of outer involutions in $\operatorname{Aut}(L)$, so $V \subset L$. Thus the lemma follows as $G=V L$.

Lemma 4.7. $L$ is not $J_{3}$ or $O^{\prime} N$.

Proof. Assume otherwise. By Lemma 4.6, $G=L$. Now $L$ has a unique class of involutions $2 A$, and Lemma 3.3 shows that the hypotheses of Lemma 4.2 (1) hold for $a \in \mathcal{A}=2 A$ and some $x \in \mathcal{O}$, completing the proof.

Lemma 4.8. $L$ is not $M_{12}$.

Proof. Assume $L \cong M_{12}$. By Lemma 4.6, $G=L$. Let $x \in 5 A, a \in 2 A$, and $b \in 2 B$. Then $C_{L}(x) \cong \mathbb{Z}_{2} \times \mathbb{Z}_{5}$ and $C_{L}(a) \cong \mathbb{Z}_{2} \times S_{5}$. By 3.4, we may assume that $a \rightsquigarrow x$ and $b \rightsquigarrow x$. Then by 4.3(1), $V \cap 2 A=\emptyset$. Consequently $V^{\#} \subset 2 B$. But by 3.4, $\operatorname{Inv}\left(C_{L}(x)\right) \subseteq 2 A$, so Lemma 4.2 (2) supplies a contradiction.

Lemma 4.9. $L$ is not $M_{22}$.

Proof. Assume $L \cong M_{22}$. By Lemma 3.2 and Lemma 4.2(1), $V \cap 2 C=\emptyset$. Next, $2 A$ is the unique class of involutions in $L$, and $x \in 5 A$ is inverted in $L$, so $2 A \rightsquigarrow 5 A$. Further $5 \notin \pi\left(C_{L}(i)\right)$ for $i \in 2 A \cup 2 B$, so $\operatorname{Inv}\left(C_{\text {Aut }(L)}(x)\right) \subset 2 C$. Thus applying Lemma 4.2 (2), $V \cap 2 A=\emptyset$, contrary to 4.4 .

Lemma 4.10. $L$ is not $M_{24}$.

Proof. Assume $L \cong M_{24}$. Then $\operatorname{Out}(L)=1$, so $G=L$. An element of order 11 is self centralizing in $L$, so by 3.1(1c) and 4.2(1), $V \cap 2 B=\emptyset$. Let $x \in 3 A$. Then $N:=N_{L}(x) \cong S_{3} \times L_{3}(2)$, so if $a \in \operatorname{Inv}(N)$ with $C_{N}(a, x) \cong L_{3}(2)$, then $a$ inverts $x$ and $a \in 2 A$ because $7 \in \pi\left(C_{L}(a)\right)$ but $C_{L}(b)$ is a $7^{\prime}$-group for $b$ in the remaining class $2 B$ of involutions. It follows from Lemma 4.2(1) that $V \cap 2 A=\emptyset$, contradicting $\operatorname{Inv}(L)=2 A \cup 2 B$.

Lemma 4.11. $L$ is not $J_{2}$.

Proof. Assume $L \cong J_{2}$. Let $x \in 7 A$. Then $N_{L}(x)$ is Frobenius of order 42 , while $\operatorname{Aut}(L)$ has a unique class $2 C$ of outer involutions, and for $c \in 2 C, C_{L}(c) \cong$ $\mathrm{PGL}_{2}$ (7). In particular we may choose $[x, c]=1$ and for $b \in \operatorname{Inv}(L)$ inverting $x$, $c b \notin L$, so $c b \in 2 C$. Thus $2 C \rightsquigarrow 7 A$, so $2 C \cap V=\emptyset$ by 4.3 (1). Therefore $G=L$.

Next, 3.3 together with Lemma $4.2(1)$ imply that $V \cap 2 B=\emptyset$. Finally by 3.5 and Lemma 4.2(2), $V \cap 2 A=\emptyset$, which is impossible since $\operatorname{Inv}(L)=2 A \cup 2 B$. 
Lemma 4.12. $L$ is not $J_{4}$.

Proof. Assume $L \cong J_{4}$. Then $\operatorname{Out}(L)=1$, so $G=L$. By Lemma 3.3 and Lemma 4.2(1), $V \cap 2 B=\emptyset$.

Next from observation (3) in the treatment of $J_{4}$ in $3.3,2 A \rightsquigarrow 11 B$, while observations (1) and (2) in that treatment imply that $\operatorname{Inv}\left(C_{L}(y)\right) \subset 2 B$, for $y \in 11 B$. Thus $V \cap 2 A=\emptyset$ by $4.2(2)$, contradicting $\operatorname{Inv}(L)=2 A \cup 2 B$.

Lemma 4.13. $L$ is not $\mathrm{Co}_{1}$.

Proof. Assume $L \cong C o_{1}$. Then $\operatorname{Out}(L)=1$, so $G=L$.

Let $z \in 2 A$ and $H:=C_{L}(z)$. By 25.6 in [A8], $\operatorname{Inv}\left(O_{2}\left(C_{L}(z)\right)\right) \subseteq 2 A$, so by $1.10(2), 2 A \cap V \neq \emptyset$.

Let $x \in 3 D$. Then $N_{L}(x) \cong S_{3} \times A_{9}$, so there is an involution $a$ inverting $x$ with $C_{L}(a, x) \cong A_{9}$. Then $3^{4}$ divides $\left|C_{L}(a)\right|$, so from the structure of centralizers of involutions in $L$ it follows that $a \in 2 A$. Then since $C_{L}(a, x) \cong A_{9}$, Lemma 4.2 (1) implies that $V \cap 2 A=\emptyset$, a contradiction.

Lemma 4.14. $\mathrm{L}$ is not $\mathrm{Co}_{2}$.

Proof. Assume $L \cong \mathrm{Co}_{2}$. Then $\operatorname{Out}(L)=1$, so $G=L$.

Let $x \in 3 B$, then $M:=N_{L}(x) \cong S_{3} \times \operatorname{Aut}\left(\operatorname{PSp}_{4}(3)\right)$. Thus if $a \in \operatorname{Inv}(M)$ with $C_{M}(a, x) \cong \operatorname{Aut}\left(\mathrm{PSp}_{4}(3)\right)$, then $a$ inverts $x$ and as $C_{L}(a)$ has an $\operatorname{Sp}_{4}(3)$ section, $a \in 2 A$. It follows from Lemma 4.2(1) that $V \cap 2 A=\emptyset$. Similarly by 3.6 (2) there exists $b \in 2 B$ inverting $x \in 5 B$ such that $C_{L}(b, x) \cong S_{5}$, so by Lemma 4.2(1), $V \cap 2 B=\emptyset$. Finally by 3.6 (3), there is $c \in 2 C$ inverting $z \in 7 A$ with $\operatorname{Inv}\left(C_{L}(c, z)\right) \subseteq 2 A \cup 2 B$, so $V \cap 2 C=\emptyset$ by 4.2 (2), a contradiction.

Lemma 4.15. $\mathrm{L}$ is not $\mathrm{Co}_{3}$.

Proof. Assume $L \cong \mathrm{Co}_{3}$. Since $\operatorname{Out}(L)=1, G=L$. Let $t \in 2 A$. Then $t$ is a 2-central involution in $L$ and $C_{L}(t) \cong 2 \operatorname{Sp}_{6}(2)$, so by $1.10(2), V \cap 2 A \neq \emptyset$. Let $x \in 5 B$, then $N:=N_{L}(x) \cong F_{20} \times A_{5}$, and hence for $a \in N$, with $C_{N}(a, x) \cong A_{5}$, $a \in 2 A$, because $a$ is a square in $L$. But now 4.2(2) says that $V \cap 2 A=\emptyset$, a contradiction.

Lemma 4.16. $L$ is not $H S$.

Proof. Assume $L \cong H S$. From the proof of 3.6(2), $x \in 5 B$ is inverted by $a \in 2 A$ with $C_{L}(a, x) \cong A_{5}$, and from [GLS3], $C_{\mathrm{Aut}(L)}(a, x) \cong S_{5}$. Thus by Lemma 4.2 (1), $V \cap 2 A=\emptyset$.

Next by Lemma 3.3 there exists $b \in 2 B$ with $b \rightsquigarrow y \in 7 A$. Now $C_{\text {Aut }(L)}(y)$ contains a unique involution $d$, and $S:=C_{L}(d) \cong S_{8}$. Then $C_{L}(d, b) \cong E_{4} \times S_{4}$, 
so $E\left(C_{L}(b)\right)=\left[E\left(C_{L}(b)\right), d\right]$. Hence by Lemma $4.3(2), V \cap 2 B=\emptyset$. Now Lemma 4.4 supplies a contradiction.

Lemma 4.17. $L$ is not $M c L$.

Proof. Let $a \in 2 A$. Then $C_{L}(a) \cong 2 A_{8}=F^{*}\left(C_{\text {Aut }(L)}(a)\right)$. By Lemma 1.10 (2), $V \cap 2 A \neq \emptyset$. By 3.3, $a \rightsquigarrow x \in 5 B$, and $O_{2}\left(C_{\text {Aut }(L)}(x, a)\right)=1$. Hence, from Lemma 4.2(1), it follows that $V \cap 2 A=\emptyset$, a contradiction.

Lemma 4.18. L is not Suz.

Proof. Let $z \in 2 A$ and $H:=C_{L}(z)$. Then $z$ is a 2-central involution and $Q=$ $\mathrm{O}_{2}(H)=F^{*}\left(C_{\mathrm{Aut}(L)}(z)\right) \cong Q_{8}^{3}$, with $H / Q \cong \Omega_{6}^{-}(2)$ transitive on the involutions $i \in Q-\langle z\rangle$, so $\left|C_{H}(i)\right|_{2}=\left(|H|_{2}\right) / 2=2^{12}$. Thus as $\left|C_{L}(b)\right|_{2}<2^{12}$ for $b \in 2 B$, $\operatorname{Inv}(Q) \subseteq 2 A$, so $V \cap 2 A \neq \emptyset$ by 1.10 (2).

By [GLS3], $H$ contains $x \in 5 A$. As all involutions in $C_{L}(x)$ are in $E\left(C_{L}(x)\right) \cong$ $A_{6}$, it follows that $z \rightsquigarrow y \in C_{L}(x)$ of order 5. Let $D:=\langle z, y\rangle$. Then $C_{L}(D) \cap N_{L}(x)$ contains an $F_{20}$-subgroup, so it follows from the structure of centralizers of elements of order 5 in $L$ that $F^{*}\left(C_{L}(D)\right)=E\left(C_{L}(y)\right)$. But now $2 A \cap V=\emptyset$ by $4.2(1)$, a contradiction.

Lemma 4.19. L is not He.

Proof. Assume $L \cong H e$. By 4.4, $V \cap L \neq 1$, while by 3.7 and 4.2(1), neither of the two classes $2 A$ and $2 B$ of involutions in $L$ intersect $V$ nontrivially.

Lemma 4.20. L is not Ru.

Proof. Assume $L \cong R u$. Since $\operatorname{Out}(L)=1, G=L$. By Lemma 3.3 and Lemma 4.2(1), $V \cap 2 B=\emptyset$.

Let $x \in 5 B$. From [GLS3], $N:=N_{L}(x) \cong F_{20} \times A_{5}$. If $a \in N$ is an involution such that $C_{L}(a, x) \cong A_{5}$ and $a$ inverts $x$ then $a \in 2 A$ since $\left|C_{L}(t)\right|$ is not divisible by 3 for $t \in 2 B$. Hence by 4.2(1), $V \cap 2 A=\emptyset$, contradicting $\operatorname{Inv}(L)=2 A \cup 2 B$.

Lemma 4.21. Assume $(G, V)$ is a minimal counter example to Theorem 2 , such that $L=F^{*}(G)$ is sporadic. Then $L$ is $F_{5}, F_{2}, F_{1}$, or one of the three Fischer groups $F_{22}, F_{23}$, or $F_{24}$.

Proof. The remaining 20 sporadics were eliminated in earlier lemmas in this section. 


\section{Some subgroups of the Monster}

In this section $M$ is the Monster $F_{1}$. In addition to our usual appeals to [GLS3], we also appeal to information contained in [GMS]. Our notation for the Fischer groups comes from [A9].

First by [GLS3]:

Lemma 5.1. $M$ has two classes $2 A$ and $2 B$ of involutions, and for $a \in 2 A, C_{M}(a) \cong$ $2 F_{2}$ is quasisimple, while for $z \in 2 B, C_{M}(z) \cong C o_{1} / D_{8}^{12}$.

Lemma 5.2. Let $a \in 2 A$. Then:

(1) For $b \in 2 A,|a b| \leq 6$.

(2) Let $3 A_{a}=\left\{b \in 2 A: C_{M}(a, b) \cong F_{23}\right\}$. Then $3 A_{a} \neq \emptyset$ and $C_{M}(a)$ is transitive on $3 A_{a}$.

(3) Let $b \in 3 A_{a}, x:=a b$, and $H:=C_{M}(x)$. Then $x \in 3 A, H \cong F_{24} / \mathbb{Z}_{3}$ is quasisimple, and for $t \in \operatorname{Inv}(H)$ with $C_{H}(t) \cong \operatorname{Aut}\left(F_{22}\right) / \mathbb{Z}_{2}, t \in 2 A$.

(4) $2 B \rightsquigarrow 41 A$, and for $y \in 41 A,\langle y\rangle$ is self centralizing in $M$.

(5) Let $w \in 5 A$. Then $C_{L}(w)=\langle w\rangle \times K$, where $K \cong F_{5}$. Further for $t \in \operatorname{Inv}(K)$ with $C_{K}(t) \cong \operatorname{Aut}(H S) / \mathbb{Z}_{2}$, we have $t \in 2 A$.

Proof. Part (1) is a consequence of 3.4.9 and 3.7 in [GMS]. Part (2) is 3.4.8 in [GMS].

Let $x_{0} \in 3 A$ and $H_{0}:=C_{M}\left(x_{0}\right)$. From [GLS3] it follows that $H_{0} \cong F_{24} / \mathbb{Z}_{3}$ and there is $a_{0} \in \operatorname{Inv}(M)$ inverting $x_{0}$ and inducing a 3-transposition on $H_{0}$. Thus $C_{M}\left(x_{0}, a_{0}\right) \cong F_{23}$, so $a_{0} \in 2 A$ and $a_{0} x_{0} \in 3 A_{a_{0}}$. Thus the first two remarks in (3) hold. The third remark appears in 3.6.3 of [GMS].

Let $y \in 41 A$. From [GLS3], $\langle y\rangle$ is self centralizing in $M$ and inverted by some involution $i$. By (1), $i \notin 2 A$, so $i \in 2 B$, establishing (4).

Finally, if $w \in 5 A$ then $C_{L}(w)=\langle w\rangle \times K$ by [GLS3], where $K \cong F_{5}$. Pick $t$ as in (5) and let $J:=C_{M}(t)$ and $J^{*}:=J /\langle t\rangle$. Then $C_{J^{*}}\left(w^{*}\right)=\left\langle w^{*}\right\rangle \times C_{K}(t)^{*}$, so from the centralizers of elements of order 5 in $C o_{1}, t \notin 2 B$. Thus $t \in 2 A$, establishing (5).

Lemma 5.3. Let $L \cong F_{2}, a \in 2 A, b \in 2 B$, and $d \in 2 D$. Then:

(1) $a^{L}$ is a class of $\{3,4\}$-transpositions of $L$.

(2) $a \rightsquigarrow x \in 3 A$ with $C_{L}(a, x) \cong \operatorname{Aut}\left(F_{22}\right)$.

(3) $b \rightsquigarrow y \in 5 A$ with $C_{L}(b, y) \cong \operatorname{Aut}(H S)$.

(4) $\operatorname{Inv}\left(O_{2}\left(C_{L}(b)\right)\right) \subseteq 2 A \cup 2 B \cup 2 D$.

(5) $d \rightsquigarrow z \in 19 A$ with $C_{L}(d, z)=\langle u\rangle$, where $u \in 2 A$. 
Proof. Part (1) is well known, see [S, (3.14)], and it appears with (2) in the Notes for $F_{2}$ in [GLS3]. Let $y \in 5 A$. By [GLS3] $N_{L}(y) \cong F_{20} \times \operatorname{Aut}(H S)$, so there is $i \in \operatorname{Inv}(L)$ inverting $y$ with $C_{L}(i, y) \cong \operatorname{Aut}(H S)$. By (1), $i \notin 2 A$, so as $11 \notin \pi\left(C_{L}(i)\right)$ for $i \in 2 C \cup 2 D$, (3) holds.

Part (4) appears in 3.6.3 in [S]. Let $z \in 19 A$. From [GLS3], $N_{L}(z)=\langle u\rangle \times F$, where $u$ is an involution and $F$ is Frobenius of order 19.18. As $19 \in \pi\left(C_{L}(u)\right)$, $u \in 2 A$. Let $i \in \operatorname{Inv}(F)$; we claim that $\{i, i u\} \cap 2 D \neq \emptyset$. Suppose not and let $Y:=C_{L}(u)$. If $i \in Y^{\prime}$, then by 3.6.2 in [S], $\{i, i u\}=\{2 A, 2 B\}$, while if $i \in Y-Y^{\prime}$, then $\{i, i u\}=\{2 A, 2 C\}$ by 3.18.1 in [S]. In either case we get a contradiction from (1). Thus (5) holds.

Lemma 5.4. Let $L \cong F_{5}$ and $A=\operatorname{Aut}(L)$. Then:

(1) Let $c$ be an involution in $A-L$. Then $c \rightsquigarrow x \in 3 A$ and $N_{A}(x)=\langle c, x\rangle \times S$ with $S \cong S_{9}$.

(2) Let $s \in S$ be a transposition. Then $a=c s \in 2 A$ inverts $x$ and $C_{L}(a, x) \cong S_{7}$.

(3) $2 B \rightsquigarrow 11 A$, and for $y \in 11 A, C_{L}(y)=\langle y\rangle \times\langle u\rangle$, with $u \in 2 A$.

Proof. Let $x \in 3 A$. By [GLS3], $N_{L}(x)$ is of index 2 in $S_{3} \times S_{9}$. Hence by a Frattini argument, either $N_{A}(x)=T \times S$ with $T \cong S_{3}$ and $S \cong S_{9}$, or $C_{A}\left(C_{L}(x)\right)$ is of order 2. The former holds as $L$ is transitive on involutions in $A-L$, and for such an involution $c, C_{L}(c) \cong S_{10}$. Thus (1) holds. Then by (1), $C_{L}(a, x) \cong S_{7}$, so as $C_{L}(b)$ has no $A_{7}$-section for $b \in 2 B$, (2) holds.

By 5.2(5), we may take $L=K \leq M$ as in that lemma, and pick $t$ as in the lemma. By the lemma, $t$ is in the $M$-class $2 A$, so by $5.2(1),\left|t t^{l}\right| \leq 6$ for $l \in L$. But by construction, $t$ is in the $L$-class $2 A$, so $t$ inverts no element of $L$ of order 11 . Then (3) follows from [GLS3].

Lemma 5.5. Assume $(G, V)$ is a minimal counter example to Theorem 2 , such that $L=F^{*}(G)$ is sporadic. Then $L$ is one of the three Fischer groups $F_{22}, F_{23}$, or $F_{24}$.

Proof. Assume otherwise. Then by 4.21, $L$ is $F_{1}, F_{2}$, or $F_{5}$. Suppose that $L \neq G$. If $L$ is $F_{1}$ or $F_{2}$, then $\operatorname{Out}(L)=1$, so $L \cong F_{5}$. But by 5.4(1) and 4.2(1), $c \notin V$, for any involution $c \in G-L$, contradicting $G=L V$. Thus $G=L$.

Let $I$ be the set of involutions fused into $O_{2}\left(C_{L}(b)\right)$ for $b \in 2 B$ if $L=F_{2}$, and let $I=\operatorname{Inv}(L)$ otherwise. By 4.4 and $1.10(2), V \cap I \neq \emptyset$. Then by 5.2-5.4, for $i \in I$, either there exists $x$ of odd prime order with $i \rightsquigarrow x$ and $O_{2}\left(C_{L}(i, x)\right)=1$, or $L \cong F_{5}$ and $i \in 2 B$, or $L \cong F_{2}$ and $i \in 2 D$. We conclude from 4.2 (1) that $L \cong F_{5}$ or $F_{2}$ and $V^{\#} \subseteq 2 B$ or $2 D$, respectively. Now by 5.4 (3) and 5.3(5), $i \rightsquigarrow y \in 11 A$ or $19 A$ with $C_{L}(y, i)=\langle u\rangle$, and $u \in 2 A$, for $L \cong F_{5}$ or $F_{2}$, respectively. Now 4.2 (2) supplies a contradiction and completes the proof. 


\section{The Fischer groups}

In this section $L$ is one of the three Fischer groups $F_{n}, n \in I=\{22,23,24\}$. Then $L=F^{*}(M)$, where $M=M(n)$ is a group generated by a set $D$ of 3-transpositions. Indeed $M=L$ unless $n=24$, where $|M: L|=2$ and $M=\operatorname{Aut}(L)$.

In addition to our usual appeals to [GLS3], we also appeal to the description of the 3-transposition group $M$ in [A9]. Further we adopt Fischer's standard notation for 3-transposition groups. For example if $d \in D$, then $d^{\perp}=C_{D}(d), D_{d}=d^{\perp}-\{d\}$, and $A_{d}=D-d^{\perp}$. A subgroup $K$ of $M$ is a $D$-subgroup if $K=\langle K \cap D\rangle$.

Lemma 6.1. (1) $M$ has $k$ classes of involutions, $j_{m}, 1 \leq m \leq k$, where $k=3$ with $n=22$ or $23, k=4$ if $n=24$, and $j_{m}$ consists of the products of $m$ commuting members of $D$.

(2) $j_{m}=2 A, 2 B, 2 C$ for $m=1,2,3$ if $n=22$ or 23 , while $j_{m}=2 C, 2 A, 2 D$, $2 B$, for $m=1,2,3,4$, if $n=24$.

(3) For $d \in D$ and $a \in A_{d}, x=a d \in 3 A$, and $F^{*}\left(C_{\mathrm{Aut}(L)}(x)\right)$ is a simple orthogonal group over $\mathbb{F}_{3}$.

(4) If $n=24$, then $j_{4} \rightsquigarrow 29 A$ and for $y \in 29 A,\langle y\rangle$ is self centralizing in $M$.

(5) If $n=22$, then $j_{3} \rightsquigarrow 13 A$ and for $z \in 13 A,\langle z\rangle$ is self centralizing in $\operatorname{Aut}(L)$.

(6) Let $n=22$ and $u \in j_{2}$. Then $u \rightsquigarrow w \in 5 A$ with $C_{\mathrm{Aut}(L)}(u, w)=C_{L}(u, w) \times$ $\langle t\rangle$, where $C_{L}(u, w) \cong S_{5}$ and $t \in 2 D$ with $\left\langle t^{C_{L}(u)}\right\rangle$ not elementary abelian.

(7) If $n=24$, then $b \in j_{2}$ inverts $w \in 5 A$ with $C_{M}(b, w) \cong S_{9}$.

Proof. Part (1) is 37.4 in [A9]. Then (2) follows from the tables in Chapter 15 of [A9]. Part (3) is part of the standard theory of 3-transposition groups; cf. 15.11 and 15.14 in [A9].

Assume $n=24$. Then by 5.2(3), the covering group $\hat{L}$ of $L$ is embedded as a subgroup of the Monster $F$ in such a way that an involution $j \in \hat{L}$, whose image in $L$ is in $j_{2}$, is in the $F$-class $2 A$. Thus by $5.2(1),\left|j j^{l}\right| \leq 6$ for $l \in L$. Thus $y \in 29 A$ is not inverted by $j$. But from [GLS3], there is an involution $i \in L$ with $i \rightsquigarrow y$, so as $\operatorname{Inv}(L) \subseteq j_{2} \cup j_{4}, i \in j_{4}$. Further $\langle y\rangle$ is self centralizing in $\operatorname{Aut}(L)$, so (4) holds.

Suppose $W \leq M$ has order 5. Then $N_{M}(W)=U \times Y$ where $U$ is Frobenius of order 20 and $Y \cong S_{9}$, with $Y \cap D$ the set of transpositions in $Y$. In particular the product $b$ of two commuting members of $Y \cap D$ inverts $w^{\prime}$ of order 5 in $Y$, and $N_{M}(W) \cap C_{M}\left(w^{\prime}, b\right)=U \times C_{Y}\left(w^{\prime}, b\right)$, with $C_{Y}\left(w^{\prime}, b\right) \cong S_{5}$. Thus $b$ centralizes $O^{2^{\prime}}\left(C_{M}\left(w^{\prime}\right)\right)$, so (7) holds.

Assume next that $n=22$. By 39.1 in [A9], there is a $D$-subgroup $K$ of $L$ isomorphic to $\Omega_{7}(3)$. Let $W$ be the natural $\mathbb{F}_{3} K$-module and $X$ a Levi factor of the maximal parabolic of $K$ stabilizing a totally singular 3-subspace of $W$. Then $X \cong L_{3}(3)$ stabilizes a decomposition $W=W_{0} \oplus W_{1} \oplus W_{2}$, where $W_{0}$ is a point 
and $W_{1}$ and $W_{2}$ are totally singular 3-subspaces. Now $X$ contains $z \in 13 A$ and $N_{X}(z)$ contains $g$ of order 3 such that $W_{i}$ is a Jordan block of size 3 for $g$ for $i=1,2$. Then, in the notation of Section 38 of [A9], $W \theta / W \theta^{2}$ is of rank 2 with singular points $W_{i} \theta / W_{i} \theta^{2}$, so $g$ is in the class $3_{+}^{2}$ of $K$ appearing in 38.15 in [A9]. Then by 39.7 in [A9], $C_{D}(g)=\emptyset$. But for $d \in D$ and $b \in D_{d}, d b \in j_{2}$ and $O^{2}\left(C_{L}(d b)\right) \leq$ $C_{L}(d)$, so $\operatorname{Inv}\left(C_{L}(g)\right) \subseteq j_{3}$. Therefore as $g$ centralizes an involution $i$ inverting $z$, it follows that $j_{3} \rightsquigarrow 13 A$, and (5) follows.

Next for $t \in 2 D, C_{L}(t)$ is a $D$-subgroup isomorphic to $\operatorname{Aut}\left(\Omega_{8}^{+}(2)\right)$. Let $L_{t} \leq$ $C_{L}(t)$ with $L_{t} \cong O_{8}^{+}(2)$ and let $U$ be the natural module for $L_{t}$. Write $U=U_{1} \perp U_{2}$ where the $U_{i}$ are nondegenerate 4-subspaces of sign -1 . Then the stabilizer in $L_{t}$ of $U_{1}$ is $L_{1} \times L_{2}$ where $L_{i}=C_{L_{t}}\left(U_{3-i}\right) \cong O\left(U_{i}\right) \cong O_{4}^{-}(2)$. In particular there is $w_{i}$ of order 5 in $L_{i}$ inverted by $u_{i}=a_{i} b_{i}$, the product of commuting 3transpositions, so $u_{i} \in j_{2}$. Then $N_{L_{t}}\left(u_{i}\right)=N_{L}\left(u_{i}\right) \cong F_{20} \times S_{5}$. Therefore $C_{\text {Aut }(L)}\left(u_{i}, w_{i}\right)=C_{L}\left(u_{i}, w_{i}\right) \times\langle t\rangle$. Finally suppose $T=\left\langle t^{C_{L}\left(u_{i}\right)}\right\rangle$ is elementary abelian. Then $T=\left\langle t, a_{i}, b_{i}\right\rangle \cong E_{8}$ as $\left\langle a_{i}, b_{i}\right\rangle$ is the maximal normal elementary abelian subgroup of $C_{L}\left(u_{i}\right)$. But then $C_{L}\left(u_{i}\right)^{\infty} \leq C_{L}(t)$, a contradiction. This establishes (6).

Lemma 6.2. If $(G, V)$ is a minimal counter example to Theorem 2 , then $L$ is not $F_{22}$, $F_{23}$, or $F_{24}$.

Proof. Assume $L \cong F_{n}$ for some $n \in I$. By 6.1 (3) and Lemma 4.2(1), $D \cap V=\emptyset$.

Suppose $n=23$. Then for $d \in D, d$ is 2-central in $G$ and $\langle d\rangle=O_{2}\left(C_{L}(d)\right)$, so $V \cap D \neq \emptyset$ by 1.10 (2), contrary to the previous paragraph. Therefore $n=22$ or 24. By 4.4, $V \cap L \neq 1$, so as $V \cap D=\emptyset$, it follows from 6.1 that $V \cap X \neq \emptyset$ for $X \in\left\{j_{2}, j_{3}\right\}, X \in\left\{j_{2}, j_{4}\right\}$ for $n=22,24$, respectively. Then it follows from parts (4), (5), and (7) of 6.1 and 4.2 (1), that $n=22$ and $V \cap L^{\#} \subseteq j_{2}$. Then 6.1 (6) and 4.3 (2) supply our final contradiction.

Notice that Lemma 5.5 and Lemma 6.2 complete the proof of Theorem 4.1.

\section{Maximal parabolics}

In this section $p$ is a prime and $L$ is a group of Lie type in characteristic $p$ of Lie rank $l \geq 1$. That is $L / O_{p}(L)$ is the central product of factors which are quasisimple groups of Lie type and characteristic $p,(S) L_{2}(3)$ if $p=3$, or $L_{2}(2),(S) U_{3}(2),{ }^{2} B_{2}(2)$, $D_{10}$, or ${ }^{2} F_{4}(2)^{\prime}$ if $p=2$. Write $R(P)$ for the unipotent radical of a parabolic $P$ of $L$. Thus $R(P)=O_{p}(P)$.

Lemma 7.1. If $L=O^{p^{\prime}}(L)$ then $L$ is not contained in a group of Lie type of characteristic $p$ and Lie rank less than $l$. 
Proof. Assume the embedding $L \leq G$ is a counter example with $l$ minimal. Then the Lie rank $k$ of $G$ is less than $l$. If $l=1$, then $k=0$, so $O^{p^{\prime}}(G)=O_{p}(G)$, impossible as $L=O^{p^{\prime}}(L)$ is of Lie rank 1 , so $O_{p}(L) \neq L$. Thus $l>1$. Passing to $G / R(G)$, we may assume $G$ is reductive.

Let $P$ be a maximal parabolic of $L, R=R(P)$, and $X=O^{p^{\prime}}(P)$. By the Borel-Tits Theorem (cf. 3.1.3 in [GLS3]), there exists a proper parabolic $Q$ of $G$ such that $P \leq Q$ and $R \leq R(Q)$. Set $Q^{*}=Q / R(Q)$ and $Y=O^{p^{\prime}}(Q)$. Then $Y^{*}$ and $X^{*}$ are of Lie type and characteristic $p$. Further $X^{*}=O^{p^{\prime}}\left(X^{*}\right)$ is reductive and as $P$ is maximal, the Lie rank of $X^{*}$ is $l-1$. As $l>1, l-1 \geq 1$. Further the Lie rank $r$ of $Y^{*}$ is at most $k-1$, so $r<l-1$. This is contrary to the minimal choice of $l$, so the lemma is established.

For $X \leq L$, let $\mathcal{P}(X)$ be the set of proper parabolic subgroups of $L$ containing $X$.

Lemma 7.2. Assume $L$ is reductive and $l>1$. Let $X=O^{p^{\prime}}(X)$ be a subgroup of $L$ of Lie type and Lie rank $l-1$. Then:

(1) For each $P \in \mathcal{P}(X), P$ is a maximal parabolic and $O_{p}(X) \leq R(P)$.

(2) If $O_{p}(X) \neq 1$, then $\mathcal{P}(X)=\{P(X)\}$ is a set of size one, $P(X)$ is a maximal parabolic, and $O_{p}(X) \leq R(P(X))$.

(3) If $|\mathcal{P}(X)|>1$, then $O_{p}(X)=1$, and for all distinct $P, Q \in \mathcal{P}(X), P$ and $Q$ are opposite maximal parabolics.

Proof. Suppose $P \in \mathcal{P}(X)$ and set $Y=O^{p^{\prime}}(P), R=R(P)$, and $Y^{*}=Y / R$. Then $X^{*}=O^{p^{\prime}}\left(X^{*}\right)$ is of Lie rank $l-1$ and $Y^{*}$ is of Lie rank at most $l-1$, so by 7.1, $Y^{*}$ is of rank $l-1$, so $P$ is a maximal parabolic. Suppose $O_{p}\left(X^{*}\right) \neq 1$. Then by Borel-Tits, there is a proper parabolic $P_{0}$ of $P$ such that $X \leq P_{0}$ and $S:=O_{p}(X) \leq R\left(P_{0}\right)$. But then $O^{p^{\prime}}\left(P_{0} / R\left(P_{0}\right)\right)$ is of Lie rank less then $l-1$, contrary to 7.1. Thus $O_{p}\left(X^{*}\right)=1$, so $O_{p}(X) \leq R$, establishing (1).

Next suppose (2) fails, and choose a counter example $X$ such that $S$ is maximal (recall $S=O_{p}(X)$ ). Let $K=N_{L}(S)$. By Borel-Tits, there exists $P \in \mathscr{P}(K)$ with $S \leq R=R(P)$. To complete the proof of (2), it suffices to show $\mathcal{P}(X)=\{P\}$, so assume otherwise and let $Q \in \mathcal{P}(X)-\{P\}$ and $T=R(Q) \cap P$. Then $X$ acts on $T$, so $X T=O^{p^{\prime}}(X T)$ is of Lie type with $P, Q$ distinct members of $\mathcal{P}(X T)$. Therefore $T=S$ by maximality of $S$.

Suppose $S=R(Q)$. By (1), $P$ and $Q$ are maximal parabolics, so as $S=R(Q)$ we have $Q=K$. Then reversing the roles of $P$ and $Q$, by symmetry $S=R \cap Q$, and as $P \neq Q, S \neq R$. But then $S<N_{R}(S) \leq R \cap Q=S$, a contradiction.

Therefore $S \neq R(Q)$, so $S<N_{R(Q)}(S) \leq R(Q) \cap P=S$. This contradiction establishes (2).

Finally suppose $P, Q$ are distinct members of $\mathcal{P}(X)$. Then $O_{p}(X)=1$ by (2). Suppose $P$ and $Q$ are not opposites. Then $1 \neq S:=R(P) \cap Q$ is $X$-invariant, so 
$Y=S X$ is of Lie type with $Y=O^{p^{\prime}}(Y)$ and $S=O_{p}(Y) \neq 1$. As $P, Q \in \mathcal{P}(Y)$, this contradicts (2). Thus (3) holds.

Let $\mathcal{T}(L)$ be the set of sets $T$ of maximal parabolic subgroups of $L$ such that for all distinct $P, Q$ in $T, P$ and $Q$ are opposites.

Lemma 7.3. Assume $L$ is reductive, $l>1$, and $X=O^{p^{\prime}}(X)$ is a reductive subgroup of $L$ of Lie type and Lie rank $l$. Let $Y$ be the set of subgroups $Y=O^{p^{\prime}}(Q)$ such that $Q$ is a maximal parabolic of $X$. Then:

(1) For each $Y \in \mathcal{Y}, \mathcal{P}(Y)=\{P(Y)\}$ is a set of size one, $P(Y)$ is a maximal parabolic, and $O_{p}(Y) \leq R(P(Y))$.

(2) Suppose $T \in \mathcal{T}(X)$. Then $S=\{P(Y): Y \in T\} \in \mathcal{T}(L)$.

Proof. Let $Y \in y$. Then $Y=O^{p^{\prime}}(Y) \leq L$ is of Lie type and Lie rank $l-1$ with $O_{p}(Y) \neq 1$, so (1) follows from $7.2(2)$.

Assume the hypothesis and notation of (2). Let $Q_{1}, Q_{2} \in T$ be distinct, and set $Y_{i}:=O^{p^{\prime}}\left(Q_{i}\right)$. Then $Y_{1,2}:=Y_{1} \cap Y_{2}=O^{p^{\prime}}\left(Q_{1} \cap Q_{2}\right)$, where $Q_{1} \cap Q_{2}$ is a common Levi factor of $Q_{1}$ and $Q_{2}$. Now $P\left(Y_{i}\right) \in \mathcal{P}\left(Y_{1,2}\right)$ for $i=1$, 2, so by 7.2 (3), either $P\left(Y_{1}\right)=P\left(Y_{2}\right)$ or $P\left(Y_{1}\right)$ and $P\left(Y_{2}\right)$ are opposites. Thus (2) holds.

Lemma 7.4. Assume $X=O^{p^{\prime}}(X)$ is a subgroup of $L$ of Lie type and characteristic $p$ of the same Lie rank as $O^{p^{\prime}}(L)$. Then $R(X) \leq R(L)$.

Proof. First $X=O^{p^{\prime}}(X) \leq O^{p^{\prime}}(L)$, and if $O^{p^{\prime}}(L)=R(L)$ the lemma is trivial, so we may assume the Lie rank of $O^{p^{\prime}}(L)$ is positive. Thus replacing $L$ by $O^{p^{\prime}}(L)$, we may assume $L=O^{p^{\prime}}(L)$, so by hypothesis, $X$ is of Lie rank $l$. Similarly replacing $L$ by $L / R(L)$, we may assume $L$ is reductive and $R=R(X) \neq 1$, and it remains to derive a contradiction.

By the Borel-Tits Theorem, there is $P \in \mathcal{P}(X)$. Now applying 7.1 to $X$ in the role of $L$, we have a contradiction.

\section{Chev $(p), p$ odd}

In this section we assume $G$ is an almost simple finite group such that $L=F^{*}(G) \in$ $\operatorname{Chev}(p)$ is of Lie type and odd characteristic $p$, and $G=L V$ for some nontrivial elementary abelian 2-subgroup $V$ of $G$.

Let $\delta=\delta(L, V)$ be the set of nonempty $V$-invariant sets $S$ of parabolic subgroups of $L$, such that for all distinct $P, Q \in S, P$ and $Q$ are opposites. In particular $O_{p}(P) \cap Q=1$. Let $\hat{\S}=\hat{\S}(L, V)$ consist of those $S \in \mathcal{S}$ such that for some $P \in S, N_{V}(P) \neq 1$. 
Lemma 8.1. Suppose $S \in \mathcal{S}$ and there exists $P \in S$ with $N_{V}(P) \neq 1$. Then there exists $(h, v) \in \mathcal{B}(G, V)$ with h a p-element, so $(G, V) \notin \mathcal{P}$.

Proof. Assume otherwise and let $R$ be the radical of $P$ and $U=N_{V}(P)$. Then $U$ is faithful on $R$, so (e.g. by 1.14 and 1.6) there is $(h, u) \in \mathscr{B}(R, U)$, and $h$ is a $p$-element. As $(h, u) \notin \mathscr{B}(G, V)$ there is $1 \neq v \in C_{V}(h)$. As $(h, u) \in \mathscr{B}(R, U)$, $v \notin U$, so $P \neq P^{v}$. Then $h=h^{v} \in R \cap R^{v}$, impossible as $P \neq P^{v}$, so $R \cap R^{v}=1$ as $P$ and $P^{v}$ are opposites.

Lemma 8.2. Assume L is of Lie rank 1. Then:

(1) The set $S$ of Borel subgroups of $L$ is in 8 .

(2) If $m_{2}(V)>2$, then $S \in \hat{s}$.

Proof. Part (1) is trivial. Suppose $S \notin \widehat{\delta}$, and let $\ell$ be the set of involutions in $\operatorname{Aut}(L)$ acting on no member of $S$. Then $V^{\#} \subseteq \mathcal{d}$. But if $L$ is ${ }^{2} G_{2}(q)$ or $U_{3}(q)$, then $d$ is empty. Hence $L \cong L_{2}(q)$. Therefore $d$ consists of the involutions in $L$ if $q \equiv-1$ mod 4 , while $d$ consists of the involutions inducing outer-diagonal automorphisms on $L$ if $q \equiv 1 \bmod 4$. In particular, $m_{2}(V) \leq m_{2}(L)=2$, completing the proof of (2).

Theorem 8.3. (1) $8(L, V) \neq \emptyset$.

(2) If $m_{2}(V) \geq 3$, then $\hat{\delta}(L, V) \neq \emptyset$.

(3) $(G, V) \notin \mathcal{P}$.

In the remainder of the section assume that $(G, V)$ is a counter example to Theorem 8.3 with $G$ of minimal order. Observe that (2), 8.1, and 1.4 imply (3), so (1) or (2) fails.

As (1) or (2) fails it follows from 8.2 that:

Lemma 8.4. The Lie rank of $L$ is greater than 1 .

Let $V \leq T \in \operatorname{Syl}_{2}(G)$. See [A2] for the definition of the set of fundamental subgroups of $L$, and the set $\operatorname{Fun}(T)$ of fundamental subgroups of $L$ associated to $T$. By 8.4 and [A2], Fun $(T)$ is nonempty and $T$-invariant. Also, from [A2] we have:

Lemma 8.5. Let $K \in \operatorname{Fun}(T)$. Then:

(1) $K \cong \mathrm{SL}_{2}(q)$, where $L$ is defined over $\mathbb{F}_{q}$.

(2) $\operatorname{Syl}_{p}(K)$ consists of centers of long root subgroups of L. Thus for $X \in \operatorname{Syl}_{p}(K)$, $N_{L}(X)$ is a proper parabolic of $L$.

(3) If $L$ is not $L_{n}(q)$ with $n>2$, then for distinct $X, Y \in \operatorname{Syl}_{p}(K), N_{L}(X)$ and $N_{L}(Y)$ are opposite maximal parabolics. 
(4) If $L$ is $L_{n}(q)$ with $n>2$, then for distinct $X, Y \in \operatorname{Syl}_{p}(K), M(X)$ and $M(Y)$ are opposite maximal parabolics, where $M(Z)$ is the stabilizer in $L$ of the center of $Z$ in the action of $L$ on its projective geometry.

Lemma 8.6. $V$ acts on no member of $\operatorname{Fun}(T)$.

Proof. Assume $V$ acts on $K \in \operatorname{Fun}(T)$ and set $S=\left\{M(X): X \in \operatorname{Syl}_{p}(K)\right\}$, where $M(X)$ is defined in 8.5 (4) if $L$ is $L_{n}(q)$, and $M(X)=N_{L}(X)$ otherwise. By 8.5, $S \in \mathcal{S}$, and if $m_{2}(V)>2$, then $1 \neq N_{V}(M(X))$ for some $X \in \operatorname{Syl}_{p}(K)$ by 8.2, so $S \in \hat{\jmath}$. But this contradicts the choice of $V$.

Lemma 8.7. $L$ is not $L_{3}^{\varepsilon}(q), G_{2}(q),{ }^{3} D_{4}(q)$, or $E_{7}(q)$.

Proof. Assume otherwise. Then by Theorem 2 in [A2], either $T$ acts on some member of Fun(T), or $L \cong G_{2}(q)$, with $q$ an odd power of 3, and $V \not \leq L$. By 8.6, the latter holds. Let $v \in V-L$ and $Y=C_{L}(v)$. Then $Y \cong{ }^{2} G_{2}(q)$. For $Q \in \operatorname{Syl}_{3}(Y)$ let $B(Q)=N_{L}\left(O^{3^{\prime}}\left(C_{L}(Z(Q))\right)\right)$. Then $B(Q)$ is a Borel subgroup of $L$. Further for $P \in \operatorname{Syl}_{3}(Y)-\{Q\}, B(P)$ and $B(Q)$ are opposites. Namely $A=R(B(Q)) \cap$ $R(B(P))$ centralizes $\langle Z(Q), Z(P)\rangle=Y$, so the remark follows as $C_{G}(Y)=\langle v\rangle$. Thus $S=\left\{B(Q): Q \in \operatorname{Syl}_{3}(Y)\right\} \in \hat{\jmath}$, contrary to the choice of $V$.

Lemma 8.8. $L$ is not $L_{n}^{\varepsilon}(q)$ or $\operatorname{PSp}_{n}(q)$ for $n>4$.

Proof. Assume otherwise and let $M$ be the natural module for $\hat{L}=\mathrm{SL}_{n}^{\varepsilon}(q)$ or $\operatorname{Sp}_{n}(q)$. For $K \in \operatorname{Fun}(T)$, let $\widehat{K}$ be the preimage of $K$ in $\hat{L}$ and $\widetilde{K}=O^{p^{\prime}}(\widehat{K})$. Then $\widetilde{K}$ is a fundamental subgroup of $\hat{L}$, and we abuse notation and write $K$ for $\widetilde{K}$. Let $\Delta=\left\{K_{1}, \ldots, K_{m}\right\}$ be an orbit of $V$ on $\operatorname{Fun}(T)$. By 8.6, $m>1$. Let $U$ be a complement to the kernel of the action of $V$ on $\Delta, D=\langle\Delta\rangle$, and $J=O^{p^{\prime}}\left(C_{D}(U)\right)$. From [A2], $[M, D]=M_{1} \perp \cdots \perp M_{m}$ and $M_{i}=\left[M, K_{i}\right]$ is the natural module for $K_{i}$. Then $J \cong \mathrm{SL}_{2}(q)$ is a full diagonal subgroup of $D$ with $[M, J]=[M, D]$, and for $X \in \operatorname{Syl}_{p}(J),[M, X]=\left[M_{1}, X\right] \perp \cdots \perp\left[M_{m}, X\right]$ is an $m$-dimensional totally singular subspace of $M$. Set $P(X)=N_{L}([M, X])$. Then $P(X)$ is a parabolic with $N_{G}(X) \leq N_{G}(P(X))$. Further for distinct $X, Y \in \operatorname{Syl}_{p}(J),[M, X] \cap C_{M}(Y)=0$, so $P(X)$ and $P(Y)$ are opposites.

As $V$ acts on $D$ and $V$ is abelian, $V$ acts on $J$. Thus $S=\{P(X): X \in$ $\left.\operatorname{Syl}_{p}(J)\right\} \in \widehat{\delta}$, contrary to the choice of $V$.

Lemma 8.9. $L$ is not $P \Omega_{n}^{\varepsilon}(q)$ for $n \geq 5$.

Proof. Assume otherwise, and let $L$ be the image in $\operatorname{PGL}(M)$ of the orthogonal group $\hat{L}$ acting on its natural module $M$, regarded as an orthogonal space over $\mathbb{F}_{q}$. Let $\Gamma=\Gamma O(M)$ be the group of semilinear maps preserving the orthogonal space $M$, 
$G^{*}(M)$ the group of similarities of $M$, and $G O(M)$ the group of isometries of $M$. These groups are discussed in Section 15 of [A1]. Further (cf. 15.1 in [A1]) as $G=L V$ for some 2-group $V, G$ is the image of some $\widehat{G} \leq \Gamma$ in $P \Gamma(M)$. As in the proof of the previous lemma, we abuse notation and identify each fundamental subgroup $K$ of $L$ with the fundamental subgroup of $\hat{L}$ mapping onto $K$ under the natural map. We recall some facts from Section 15 of [A1].

Let $K \in \operatorname{Fun}(T)$ and $z=z(K)$ the involution in $K$. Then there exists a unique $K^{\prime} \in \operatorname{Fun}(T)-\{K\}$ with $z\left(K^{\prime}\right)=z(K)$. Indeed $[M, z]$ is a 4-dimensional space of sign + and $K K^{\prime}=O^{p^{\prime}}(O([M, z]))$.

Suppose $v \in G$ is an involution with $K^{v}=K^{\prime}$, and let $v$ be the image of $\hat{v} \in \Gamma$. Then $O^{p^{\prime}}\left(C_{K K^{\prime}}(v)\right)=J \cong L_{2}(q)$. Let $X \in \operatorname{Syl}_{p}(J)$.

Assume first that $\hat{v} \in G^{*}(M)$. Then from 15.8 in [A1], the action of $v$ on $K K^{\prime}$ agrees with that of some involution in $G O(M)$, and $J$ acts as $\Omega_{3}(q)$ on $[M, z]$. Thus $[M, z]=[M, J] \perp M_{0}$ is the orthogonal direct sum of a nonsingular point $M_{0}$ with a 3-dimensional orthogonal space $[M, J], X$ is a short root subgroup of $L$, and $M(X):=C_{[M, J]}(X)=[M, X, X]$ is a singular point of $M$, so $P(X)=N_{L}(M(X))$ is a parabolic subgroup of $L$ with $N_{G}(X) \leq N_{G}(P(X))$. Further for distinct $X, Y \in$ $\operatorname{Syl}_{p}(J), M(X)$ is not orthogonal to $M(Y)$, so $P(X)$ and $P(Y)$ are opposites.

So assume instead that $\hat{v} \notin G^{*}(M)$. Then $\hat{v}=\sigma t$ where $\sigma$ induces an involutory field automorphism on $K$ and $K^{\prime}$, and $t$ is a reflection with $K^{t}=K^{\prime}$. In this case $J$

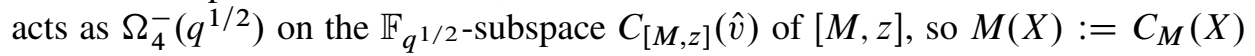
is again a singular point of $M$, and $P(X)$ has the same properties as in the previous case.

Let $\Delta=\left\{K_{1}, \ldots, K_{m}\right\}$ be an orbit of $V$ on $\operatorname{Fun}(T)$. By 8.6, $m>1$. Let $U$ be a complement to the kernel of the action of $V$ on $\Delta, D=\langle\Delta\rangle$, and $J=O^{p^{\prime}}\left(C_{D}(U)\right)$. There are two cases:

(i) For $1 \leq i \leq m, K_{i}^{\prime} \notin \Delta$.

(ii) For $1 \leq i \leq m, K_{i}^{\prime} \in \Delta$.

From the discussion above, $[M, D]=M_{1} \perp \cdots \perp M_{r}$, where $r=m$ in case (i), and in case (ii), $r=m / 2$ and we can choose notation so that $K_{i}^{\prime}=K_{i+r}$, where the indices are read modulo $r$. Further $M_{i}=\left[M, K_{i}\right]$ is a 4-dimensional orthogonal space of sign +1 , and the sum of two natural modules for $K_{i}$. In case (ii), $M_{i}=\left[M, K_{i}^{\prime}\right]$.

In case (i), $J \cong \mathrm{SL}_{2}(q)$ is a full diagonal subgroup of $D$ with $[M, J]=$ $[M, D]$, and for $X \in \operatorname{Syl}_{p}(J),[M, X]=\left[M_{1}, X\right] \perp \cdots \perp\left[M_{m}, X\right]$ is a $2 m$ dimensional totally singular subspace of $M$. Set $P(X)=N_{L}([M, X])$. Then $P(X)$ is a parabolic with $N_{G}(X) \leq N_{G}(P(X))$. Further for distinct $X, Y \in \operatorname{Syl}_{p}(J)$, $[M, X] \cap[M, Y]^{\perp}=0$, so $P(X)$ and $P(Y)$ are opposites.

In case (ii), there is $w \in U$ such that for each $1 \leq i \leq r, K_{i}^{w}=K_{i}^{\prime}$. Let $J_{i}=O^{p^{\prime}}\left(C_{K_{i} K_{i}^{\prime}}(w)\right)$. From our earlier discussion, $J_{i} \cong L_{2}(q)$. Let $E=\left\langle J_{i}\right.$ : $1 \leq i \leq r\rangle$. Then $J$ is a full diagonal subgroup of $E$. Let $X \in \operatorname{Syl}_{p}(J), X_{i}$ the projection of $X$ on $J_{i}$, and write $M\left(X_{i}\right)$ for the singular point of $M_{i}$ defined 
above. Then $M(X)=M\left(X_{1}\right) \perp \cdots \perp M\left(X_{r}\right)$ is an $r$-dimensional totally singular subspace of $M$. Set $P(X)=N_{L}(M(X))$. Again $N_{G}(X) \leq N_{G}(P(X))$, and for distinct $X, Y \in \operatorname{Syl}_{p}(J), P(X)$ and $P(Y)$ are opposites.

As $V$ acts on $D$ and $V$ is abelian, $V$ acts on $J$. Thus $S=\{P(X): X \in$ $\left.\operatorname{Syl}_{p}(J)\right\} \in \mathcal{S}$. Then as $1 \neq U \leq N_{G}(P(X)), S \in \hat{\gamma}$, contrary to the choice of $V$. This completes the proof of the lemma.

Lemma 8.10. $L$ is $F_{4}(q), E_{6}^{\varepsilon}(q)$, or $E_{8}(q)$.

Proof. By 8.3, the Lie rank of $L$ is greater than 1. Hence if $L$ is $L_{n}^{\varepsilon}(q)$ or $\operatorname{PSp}_{n}(q)$, then by $8.7, n \geq 4$. Then by $8.8, n=4$. Thus $L$ is $P \Omega_{m}^{\varepsilon}(q)$ for $m=5$ or 6 , contrary to 8.9. Thus if $L$ is classical, then $L$ is $P \Omega_{n}^{\varepsilon}(q)$ for some $n>6$, contrary to 8.9. Therefore $L$ is exceptional. Now the lemma follows from 8.7.

We recall from Definition 4.1.8 in [GLS3] that an involution $z \in L$ is of parabolic type if $O^{p^{\prime}}\left(C_{L}(z)\right)=O^{p^{\prime}}\left(P \cap P^{\prime}\right)$ for some pair $P, P^{\prime}$ of opposite maximal parabolics. Also $z$ is of equal rank type if $O^{p^{\prime}}\left(C_{L}(z)\right)$ has the same Lie rank as $L$.

Lemma 8.11. (1) L has a unique class $z^{L}$ of 2-central involutions.

(2) Let $L_{z}=C_{L}(z)$. Then either

(i) $L$ is $F_{4}(q)$ or $E_{8}(q)$, $z$ is of equal-rank type, $L_{z}$ is quasisimple, $\langle z\rangle=Z\left(L_{z}\right)$, and $L_{z}$ is $\operatorname{Spin}_{9}(q)$ or $\Omega_{16}^{+}(q)$, respectively.

(ii) $L$ is $E_{6}^{\varepsilon}(q)$, $z$ is of parabolic type, and $O^{p^{\prime}}\left(L_{z}\right) \cong \operatorname{Spin}_{10}^{\varepsilon}(q)$.

(3) We may assume $V \leq C_{G}(z)$.

Proof. Parts (1) and (2) are a consequence of Theorem 4.5.1 in [GLS3]. By (1), $z$ is 2-central in $G$, so (3) follows.

Lemma 8.12. $L$ is not $E_{6}^{\varepsilon}(q)$.

Proof. Assume otherwise. Choose $z$ as in 8.11 and let $L_{z}=C_{L}(z)$. Thus $V \leq G_{z}=$ $C_{G}(z)$. By $8.11(2), z$ is of parabolic type. Let $S=\mathcal{P}\left(O^{p^{\prime}}\left(L_{z}\right)\right)$, in the notation of Section 7. Then $S$ is $V$-invariant, and by 7.2(3), $S \in \widehat{s}$, contrary to the choice of $V$.

Lemma 8.13. $L$ is not $F_{4}(q)$ or $E_{8}(q)$.

Proof. Assume otherwise. Choose $z$ as in 8.11 and let $L_{z}=C_{L}(z)$. Thus $V \leq$ $G_{z}=C_{G}(z)$. By $8.11(2), z$ is of equal-rank type. By minimality of $|G|$, there is $S_{z} \in \hat{\gamma}\left(V L_{z}, V\right)$. Indeed from 8.11(2i), $L_{z}$ is $\operatorname{Spin}_{9}(q)$ or $\Omega_{16}^{+}(q)$, while from the treatment of these groups in 8.9, the members of $S_{z}$ are maximal parabolics of $L_{z}$. Thus for $Y \in S_{z}, \mathcal{P}(Y)=\{P(Y)\}$ is a set of size one by 7.3(1). Set $S=\left\{P(Y): Y \in S_{z}\right\}$. By 7.3(2), $S \in \hat{8}$, contrary to the choice of $V$. 
Observe that $8.10,8.12$, and 8.13 complete the proof of Theorem 8.3. Further, Theorem 8.3 implies Theorem 3, and deals with those minimal counter examples to Theorem 2 whose generalized Fitting subgroup is of Lie type and odd characteristic.

\section{Classical groups in characteristic 2}

In this section $q \geq 2$ is a power of 2 , and $M$ is an $n$-dimensional vector space over $F:=\mathbb{F}_{q}$.

Given an involution $u \in \operatorname{GL}(M)$ and $a \in F$, define

$$
U(a):=1+a(u+1) \in \operatorname{End}(M),
$$

and set

$$
R t(u)=R t_{\mathrm{GL}(M)}(u)=\{U(a): a \in F\} .
$$

Observe that:

Lemma 9.1. (1) For each involution $u \in \mathrm{GL}(M)$, the map $a \mapsto U(a)$ is an isomorphism of the additive group of $F$ with the subgroup $R t(u)$ of $\operatorname{GL}(M)$.

(2) $\operatorname{Rt}(u) \leq Z\left(C_{\mathrm{GL}(M)}(u)\right)$.

As in Section 4 of [ASe], for $1 \leq l \leq n / 2$, write $j_{l}$ for the set of all involutions $u \in \mathrm{GL}(M)$ such that $\operatorname{dim}([M, u])=l$. From 4.1 in [ASe]:

Lemma 9.2. $j_{l}, 1 \leq l \leq n / 2$, is the set of conjugacy classes of involutions in $\operatorname{SL}(M)$.

Pick $1 \leq l \leq n / 2$ and let $V, W$ be $F$-spaces of dimension $2, l$, respectively. Set $K:=\operatorname{SL}(V), L_{1}:=\operatorname{GL}(W), M_{1}:=V \otimes W$, and let $\pi: K \times L_{1} \rightarrow \operatorname{GL}\left(M_{1}\right)$ be the tensor product representation. Let $u$ be an involution in $K$ and $U$ the radical of the Borel subgroup of $K$ containing $u$. Observe that $R t_{\mathrm{GL}(V)}(u)=U$, and that an easy calculation from linear algebra shows $U \pi=R t_{\mathrm{GL}\left(M_{1}\right)}(u \pi)$.

Regard $M_{1}$ as a subspace of $M$ and choose a complement $M_{2}$ to $M_{1}$ in $M$. Extend $\pi$ to a representation $\rho: K \times L_{1} \rightarrow \mathrm{GL}(M)$ by decreeing that $g \rho_{\mid M_{2}}=1$ for $g \in K \times L_{1}$. Then as $U \pi=R t_{\mathrm{GL}\left(M_{1}\right)}(u \pi)$, also $U \rho=R t_{\mathrm{GL}(M)}(u \rho)$. Identify $K$ and $L_{1}$ with their images in GL $(M)$ under the injection $\rho$. Then $K$ centralizes $L_{1}$, and each of these subgroups centralizes

$$
L_{2}:=C_{\mathrm{GL}(M)}\left(M_{1}\right) \cap N_{\mathrm{GL}(M)}\left(M_{2}\right) .
$$

By construction, $u \in j_{l}$. Further, from 4.3 in [ASe], $L_{u}:=L_{1} L_{2} \cap \operatorname{SL}(M)$ is a Levi factor of $C_{\mathrm{SL}(M)}(u)$. Next $C_{\mathrm{GL}(M)}(K)$ acts on $M_{1}=[M, K]$ and $M_{2}=C_{M}(K)$, so $C_{\mathrm{SL}(M)}(K)=L_{2} C_{G}(K)$, where $G:=C_{\mathrm{GL}(M)}\left(M_{2}\right) \cap N_{\mathrm{GL}(M)}\left(M_{1}\right)$. By 27.14 in [A4], $L_{1}=C_{\mathrm{GL}\left(M_{1}\right)}(K)$. Together with 9.1 (2) these observations imply: 
Lemma 9.3. $u \in j_{l}$ and $(K, U) \in \mathfrak{S}(\operatorname{SL}(M), u)$.

Recall that $\mathfrak{S}(Y, i)$ was defined in the Introduction, for $Y$ a group of Lie type in characteristic 2, and $i$ an involution in $Y$. Indeed $C_{\mathrm{GL}(M)}\left(L_{2}\right)=Z\left(L_{2}\right) G$, and by 27.14 in [A4], $C_{\mathrm{GL}\left(M_{1}\right)}\left(L_{1}\right)=K Z\left(\mathrm{GL}\left(M_{1}\right)\right)$, so:

Lemma 9.4. $K Z(\operatorname{SL}(M))=C_{\mathrm{SL}(M)}\left(L_{u}\right)$.

Therefore

$$
K=K(u):=O^{2^{\prime}}\left(C_{\mathrm{SL}(M)}\left(L_{u}\right)\right)
$$

is canonically defined by $u$, up to conjugation in $C_{\mathrm{SL}(M)}(u)$.

Let $A:=\operatorname{Aut}(F)$ and pick a basis $B_{X}$ for each $X \in\left\{V, W, M_{2}\right\}$. Then $B=$ $B_{V} \otimes B_{W} \cup B_{M_{2}}$ is a basis for $M$, and if $\Sigma:=\left\{\sigma_{a}: a \in A\right\}$ is the group of field automorphisms of GL(M) determined by $B$ then $\Sigma$ acts on $K, L_{1}$, and $L_{2}$, and we may choose notation so that $\Sigma$ centralizes $u$. Further $\Sigma$ induces a group of field automorphisms on $K, L_{1}$, and $L_{2}$.

Similarly let $\tau$ be the transpose-inverse map on $\operatorname{GL}(M)$ determined by $B$. Then $\tau$ is a graph automorphism of $\operatorname{SL}(M)$ acting on $K, L_{1}$ and $L_{2}$, and $\tau$ induces an inner automorphism on $K$, and graph automorphisms on $L_{1}$ and $L_{2}$. We conclude:

Lemma 9.5. $C_{\mathrm{Aut}(\mathrm{SL}(M))}(K)=\langle t\rangle L_{u}$, where $t \in \tau L_{u}$ induces a graph automorphism on $L_{u}$.

Given a form $f$ on a vector space $N$, write $O(N, f)$ for the isometry group of the form.

Next let $f_{X}$ be a bilinear or sesquilinear form on $X$ for $X \in\left\{V, W, M_{2}\right\}$. Then $f_{1}=f_{V} \otimes f_{W}$ is the unique form on $M_{1}$ such that

$$
f_{1}\left(v_{1} \otimes w_{1}, v_{2} \otimes w_{2}\right)=f_{V}\left(v_{1}, v_{2}\right) f_{W}\left(w_{1}, w_{2}\right),
$$

for $v_{i} \in V$ and $w_{i} \in W$ (cf. Section 9 in [A3]). Indeed by parts (2) and (3) of 9.1 in [A3], if $f_{X}$ is symplectic, unitary, for $X \in\{V, W\}$, then so is $f_{1}$. Further if $f_{X}$ is symmetric or unitary and nondegenerate then by 9.1 .1 in [A3], so is $f_{1}$. If $f_{V}$ is symplectic, then

$$
f_{1}(v \otimes w, v \otimes w)=f_{V}(v, v) f_{W}(w, w)=0 \cdot f_{W}(w, w)=0,
$$

so $f_{1}$ is symplectic. Let $f_{M}=f_{1}+f_{M_{2}}$, and observe by construction $M_{1}$ is nondegenerate and $M_{2}=M_{1}^{\perp}$. We have shown:

Lemma 9.6. (1) If $f_{V}, f_{W}$, and $f_{M_{2}}$ are unitary, then so is $f_{M}$.

(2) If $f_{V}$ and $f_{M_{2}}$ are symplectic, and $f_{W}$ is symmetric and nondegenerate, then $f_{M}$ is symplectic.

(3) In either case $M_{1}$ is nondegenerate and $M_{2}=M_{1}^{\perp}$. 
If $f_{V}$ is symplectic let $K_{f}=K$, and observe that $K=\operatorname{Sp}\left(V, f_{V}\right)$ preserves $f_{V}$. If $q=q_{0}^{2}$ is a square and $f_{V}$ is unitary, let $K_{f}=K \cap O\left(V, f_{V}\right)$. Then $K_{f} \cong L_{2}\left(q_{0}\right)$ and we choose notation so that $u \in K_{f}$. Let $L_{1, f}=L_{1} \cap O\left(W, f_{W}\right)$ and $L_{2, f}=L_{2} \cap O\left(M_{2}, f_{M_{2}}\right)$. Then by 9.2 in [A3]:

Lemma 9.7. $K_{f}, L_{1, f}$, and $L_{2, f}$ are subgroups of $O\left(M, f_{M}\right)$.

First assume $f_{M}$ is unitary. Then $O\left(M, f_{M}\right)=\mathrm{GU}(M)$ is the general unitary group on $M$, and $\mathrm{SU}(M)=\mathrm{SL}(M) \cap \mathrm{GU}(M)$ is the special unitary group. We abuse notation and write $j_{m}$ for $j_{m} \cap \mathrm{GU}(M)$. By 6.1 in [ASe]:

Lemma 9.8. $j_{l}, 1 \leq l \leq n / 2$, is the set of conjugacy classes of involutions in $\mathrm{SU}(M)$, and $u \in j_{l}$.

Let $U_{f}:=U \cap K_{f}$. Thus $U_{f}$ is a Sylow 2-subgroup of $K_{f} \cong L_{2}\left(q_{0}\right)$ and from 9.1(2), $U_{f}=R t_{\mathrm{GU}(M)}(u)$, so $U_{f} \leq Z\left(C_{\mathrm{GL}(U)}(u)\right)$. By construction, $K_{f}$ centralizes $L_{i, f}$ for $i=1,2, L_{1, f}=\mathrm{GU}\left(W, f_{W}\right) \cong \mathrm{GU}_{l}\left(q_{0}\right)$, and $L_{2, f}=$ $\mathrm{GU}\left(M_{2}, f_{M_{2}}\right) \cong \mathrm{GU}_{n-2 l}\left(q_{0}\right)$. Then arguing as in the proof of 9.3, using 6.2 in [ASe] in place of 4.3 in [ASe], we conclude:

Lemma 9.9. If $u \in j_{l}$, then $\left(K_{f}, U_{f}\right) \in \mathbb{S}(\mathrm{SU}(M), u)$.

Further $\mathrm{GU}(M)$ is the centralizer in $\operatorname{GL}(M)$ of the graph-field automorphism $\tau \sigma$, where $\sigma$ is the involution in $\Sigma$. Hence $\Sigma \cong \operatorname{Out}(\operatorname{SL}(M)),\langle\sigma\rangle=C_{\Sigma}\left(K_{f}\right), \Sigma /\langle\sigma\rangle$ induces the group of field automorphisms on $K_{f}$, and (in the language of 2.5.13 in [GLS3]) $\sigma$ induces a graph automorphism on $\mathrm{SU}(M)$.

Next assume $f_{V}, f_{W}$, and $f_{M_{2}}$ satisfy the hypothesis of 9.6(2), so that $f_{M}$ is symplectic by that lemma. Then $O\left(M, f_{m}\right)=\mathrm{Sp}(M)$ is the symplectic group on $M$, and in particular contained in $\operatorname{SL}(M)$. From 7.7 in [ASe]:

Lemma 9.10. The set of conjugacy classes of involutions in $\operatorname{Sp}(M)$ is $a_{l}, c_{l}, b_{k}$, $1 \leq l, k \leq n / 2$, l even, $k$ odd.

The notation is explained in Section 7 of [ASe]. In particular $\operatorname{dim}([i, M])=l$ for $i \in x_{l}$ and $x \in\{a, b, c\}$. Thus $u \in x_{l}$ for some $x \in\{a, b, c\}$. Moreover $x \in\{b, c\}$ iff there exists $x \in M$ such that $f_{M}(x, x u) \neq 0$.

Suppose $x_{2} \in V-C_{V}(u)$ so that $x_{2} u=x_{2}+x_{1}$ for some $x_{1} \in[V, u]$ and $f_{V}\left(x_{1}, x_{2}\right) \neq 0$. Let $w \in W$. Then $\left(x_{2} \otimes w\right) u=x_{2} \otimes w+x_{1} \otimes w$, and $f_{M}\left(x_{2} \otimes\right.$ $\left.w,\left(x_{2} \otimes w\right) u\right) \neq 0$ iff

$$
0 \neq f_{M}\left(x_{2} \otimes w, x_{1} \otimes w\right)=f_{V}\left(x_{2}, x_{1}\right) f_{W}(w, w),
$$

so as $f_{V}\left(x_{1}, x_{2}\right) \neq 0, u \in a_{l}$ iff $f_{W}(w, w)=0$ for all $w \in W$ iff $f_{W}$ is symplectic. 
We now make a choice of the form $f_{W}$. We just saw that $u \in a_{l}$ iff $f_{W}$ is symplectic. Further $L_{2, f}=O\left(M_{2}, f_{M_{2}}\right)=\operatorname{Sp}\left(M_{2}\right) \cong \operatorname{Sp}_{n-2 l}(q)$, and $L_{1, f}=$ $O\left(W, f_{W}\right)=\operatorname{Sp}(W) \cong \operatorname{Sp}_{l}(q)$ as $f_{W}$ is symplectic. Then arguing as in the proof of 9.3, using 7.9 in [ASe] in place of 4.3 in [ASe], we conclude:

Lemma 9.11. If $u \in a_{l}$, then $(K, U) \in \mathfrak{S}(\operatorname{Sp}(M), u)$.

To obtain elements of $b_{l}$ and $c_{l}$ we choose $f_{W}$ to have an orthonormal basis $B_{W}$. Then $f_{W}$ is not symplectic, so by an earlier remark, $u \in b_{l}, c_{l}$ for $l$ odd, even, respectively. Set $z:=\sum_{w \in B_{W}} w$. If $l$ is odd then $z$ is nonsingular and $W=z \oplus z^{\perp}$ with $z^{\perp}$ symplectic, so $L_{1, f} \cong O\left(W, f_{W}\right) \cong \mathrm{Sp}\left(z^{\perp}\right) \cong \operatorname{Sp}_{l-1}(q)$. Then arguing as in the proof of 9.3, using 7.10 in [ASe] in place of 4.3 in [ASe], we conclude:

Lemma 9.12. If $u \in b_{l}$, then $(K, U) \in \mathbb{S}(\operatorname{Sp}(M), u)$.

Finally if $l$ is even, then $z$ is singular with $z^{\perp} /\langle z\rangle$ symplectic, so $O\left(W, f_{W}\right) \cong$ $\operatorname{Sp}_{l-2}(q) / E_{q^{l-1}}$, and hence $O_{2}\left(C_{\mathrm{Sp}(M)}(K)\right) \neq 1$. Thus in this case $(K, U) \notin$ $\mathfrak{S}(\operatorname{Sp}(M), u)$, so we must look elsewhere for members of $\subseteq(\operatorname{Sp}(M), u)$ when $u \in c_{l}$. However as we are only interested in proving Theorem 4 , we need only consider involutions in $c_{2}$.

Take $l=2$ and let $E$ be a quadratic extension of $F$. As in Section 7 of [A3], there is an $E$-structure $M_{E}$ on $M_{1}$ and a sympletic form $f_{E}$ on $M_{E}$, such that $f_{V}=\operatorname{Tr}_{F}^{E} \circ f_{E}$. Let $K_{E}=O\left(M_{E}, f_{E}\right) \cong \operatorname{Sp}_{2}(E) \cong L_{2}\left(q^{2}\right)$. Then from 7.2.6 in [A3], $K_{E} \leq \operatorname{Sp}\left(M_{1}\right)$. For $u_{E}$ an involution in $K_{E}, \operatorname{dim}\left(\left[M, u_{E}\right]\right)=2$ and $u_{E}$ inverts an element of order $q^{2}+1$, so $u_{E} \notin a_{2}$ since the root elements in $a_{2}$ only invert elements of odd order dividing $q^{2}-1$. Therefore $u_{E} \in c_{2}$, so we may take $u \in K_{E}$. Indeed if $R t_{\mathrm{SL}\left(M_{E}\right)}(u)=\left\{U_{E}(e): e \in E\right\}$ then $U=$ $R t_{K}(u)=\left\{U_{E}(a): a \in F\right\} \leq K_{E}$. Further $K_{E}$ is irreducible on $M_{1}=\left[M, K_{E}\right]$, so $C_{\mathrm{Sp}(M)}\left(K_{E}\right)=L_{2, f} \cong \operatorname{Sp}_{n-4}(q)$. Finally if $q=2$ and $u \in D \leq K_{E}$ with $D \cong D_{10}$, then $\operatorname{Sp}\left(M_{1}\right) \cong S_{6}$ so $C_{\mathrm{Sp}\left(M_{1}\right)}(D)=1$, and hence $C_{\mathrm{Sp}(M)}(D)=L_{2, f}$. Then, as usual, arguing as in the proof of 9.3, using 7.11 in [ASe] in place of 4.3 in [ASe], we conclude:

Lemma 9.13. If $u \in c_{2}$, then $\left(K_{E}, U\right) \in \mathcal{S}(\operatorname{Sp}(M), u)$.

If $n \neq 4$, then from [GLS3], $\Sigma \cong \operatorname{Out}(\operatorname{Sp}(M))$. Further $\Sigma$ induces a group of field automorphisms on $K, K_{E}, L_{1, f}$, and $L_{2, f}$. When $n=4$, $\operatorname{Out}(\operatorname{Sp}(M))$ is cyclic with $\Sigma$ of index 2, and for $t \in \operatorname{Aut}(\operatorname{Sp}(M))$ whose image is not in $\Sigma, t$ is nontrivial on the Dynkin diagram of $\operatorname{Sp}(M)$.

Finally we consider the orthogonal groups. Let $Q$ be a quadratic form on $M$ with associated symplectic form $f_{M}$. Then $O(M, Q)=O^{\varepsilon}(M)$, where $\varepsilon$ is the sign of $Q$. Note that in particular, $O^{\varepsilon}(Q) \leq \mathrm{Sp}(M)$. Now $n$ is even, and we may assume $n \geq 6$. 
Let $G:=\Omega^{\varepsilon}(M)$ be the commutator group of $O^{\varepsilon}(M)$. Then $G \cong \Omega_{n}^{\varepsilon}(q)$ is simple of index 2 in $O^{\varepsilon}(M) \cong O_{n}^{\varepsilon}(q)$. We abuse notation and write $x_{l}$ for the intersection $x_{l} \cap O^{\varepsilon}(M)$ of the class $x_{l}$ in $\operatorname{Sp}(M)$ with $O^{\varepsilon}(M)$, for $x \in\{a, b, c\}$. Then from 8.5, 8.11 , and 8.12 in [ASe]:

Lemma 9.14. (1) The set of conjugacy classes of involutions in $O^{\varepsilon}(M)$ is $a_{l}, c_{l}, b_{k}$, $1 \leq l, k \leq n / 2, l$ even, $k$ odd, with $\varepsilon=+1$ for $a_{n / 2}$.

(2) $a_{l}$ and $c_{l}$ are contained in $\Omega^{\varepsilon}(M)$, but $b_{k}$ is not.

(3) $a_{l}$ and $c_{l}$ are classes in $\Omega^{\varepsilon}(M)$, except that $a_{n / 2}$ splits into two $\Omega^{+}(M)$ classes, with $t$ and $s$ conjugate in $\Omega^{+}(M)$ iff $[M, s]$ and $[M, t]$ are conjugate.

In Theorem 4 we are only concerned with involutions in the simple group $\Omega^{\varepsilon}(M)$, which by 9.14 are of type $a_{l}$ and $c_{l}$. Further in Theorem 4 we do not need to consider $c_{l}$ for $l>2$.

First we may take $u$ of type $a_{l}$; hence $l$ is even and $f_{W}$ is symplectic. By 9.1 .4 in [A3], there is a unique quadratic form $Q_{1}$ on $M_{1}$ with associated bilinear form $f_{1}$ such that $Q_{1}(v \otimes w)=0$ for all $v \in V$ and $w \in W$. By 9.5 in [A3], $\left(M_{1}, Q_{1}\right)$ is of sign +1 . Pick a quadratic form $Q_{2}$ on $M_{2}$ of sign $\varepsilon$, and let $Q=Q_{1}+Q_{2}$. Then $Q$ is a quadratic form on $M$ of sign $\varepsilon$ with symplectic form $f_{M}$. Note if $n=2 l$, then $Q=Q_{1}$ is of sign +1 , which is forced by 9.14(1). By 9.2.4 in [A3], $K \times L_{1, f} \cong L_{2}(q) \times \operatorname{Sp}_{l}(q)$ preserves $Q$ and hence is contained in $\Omega^{\varepsilon}(M)$. Let $L_{2, Q}=\Omega\left(M_{2}, Q_{2}\right) \cong \Omega_{n-2 l}^{\varepsilon}(q)$, so that $L_{2, Q} \leq \Omega^{\varepsilon}(M)$ centralizes $K$ and $L_{1, f}$. As usual, arguing as in the proof of 9.3, using 8.6 in [ASe] in place of 4.3 in [ASe], we conclude:

Lemma 9.15. If $u \in a_{l}$, then $(K, U) \in \mathbb{S}\left(O^{\varepsilon}(M), u\right)$.

This time $\operatorname{Aut}\left(\Omega^{\varepsilon}(M)\right)$ is $\Gamma O^{\varepsilon}(M)$, which is $O^{\varepsilon}(M)$ extended by $\Sigma$, unless $\operatorname{dim}(M)=8$ and $\varepsilon=+1$, where $\operatorname{Aut}\left(\Omega^{\varepsilon}(M)\right)=\Gamma O^{\varepsilon}(M)\langle\xi\rangle$, for a graph automorphism $\xi$ of order 3. Further $\Sigma$ induces field automorphisms on $K, L_{1, f}$ and $L_{2, Q}$, and when $l=2$, a suitable transvection in $O^{\varepsilon}(M)$ centralizes $K L_{1, f}$ and induces a transvection on $L_{2, Q}$. Thus for $u=a_{2}, O_{2}\left(C_{\Gamma O^{\varepsilon}(M)}(K)\right)=1$.

Lemma 9.16. Let $u \in c_{2}, M_{*}$ a nonsingular point of $[M, u]$, and $Y:=N_{G}\left(M_{*}\right)$. Assume $q>2$ or $n>6$.

(1) There is $\left(K_{*}, U_{*}\right) \in \mathfrak{S}(Y, u)$.

(2) $\left(K_{*}, U_{*}\right) \in \mathbb{S}(G, u)$.

(3) $\left[M, K_{*}\right]$ is 3-dimensional with $f_{M}$-radical $M_{*}$, and for $x \in M=M_{*}^{\perp}, M_{x}=$ $\left\langle M_{*}, x\right\rangle$ is a nondegenerate 4-dimensional orthogonal space, such that for each involution $t_{x} \in O^{\varepsilon}(M)$ inducing a transvection on $M_{x}$ with center $M_{*}, t_{x}$ centralizes $K_{*}$. 
(4) $C_{\Gamma O^{\varepsilon}(M)}\left(K_{*} C_{G}\left(K_{*}\right)\right)=\left\langle t_{*}\right\rangle$, where $t_{*} \in O^{\varepsilon}(M)$ is the transvection with center $M_{*}$.

Proof. First, $Y$ acts faithfully as $\operatorname{Sp}(\tilde{M}) \cong \operatorname{Sp}_{n-2}(q)$ on $\tilde{M}:=M_{*}^{\perp} / M_{*}$. In particular $u$ is of type $b_{1}$ in $Y$, so (1) follows from 9.12, and from the proof of that lemma, $Y_{*}=C_{Y}\left(K_{*}\right) \cong \operatorname{Sp}_{n-4}(q)$.

Next $C_{G}(u) \leq G_{0}$, where $G_{0}$ is the stabilizer in $G$ of the unique singular point $M_{0}$ in $[M, u]$. Further $R:=R\left(G_{0}\right)$ is abelian and $C_{G}(u)=R L_{0}$ where $L_{0} \cong \operatorname{Sp}_{n-4}(q)$. Thus $Y_{*} \cong L_{0}$, so $U_{*} \leq C_{R}\left(Y_{*}\right)=C_{R}\left(L_{0}\right)=Z\left(C_{G}(u)\right)$. Let $Y_{1}:=C_{G}\left(K_{*}\right)$. Then $R_{1}:=O_{2}\left(Y_{1}\right)$ is $Y_{*}$-invariant, so as $C_{G}(u)=Y_{*} R, R_{1} \leq R$. Then as $q>2$ or $n>6$, each nontrivial $Y_{*}$-submodule of $R$ intersects $U_{*}$ nontrivially. Thus either $R_{1}=1$ or $U_{1}=U_{*} \cap R_{1} \neq 1$. In the latter case $K_{*}=\left\langle U_{1}^{K *}\right\rangle \leq R_{1}$, a contradiction. Thus (2) holds.

Further by construction, the first remark in (3) holds. Thus for $x \in M=M_{*}^{\perp}$, $M_{x}=\left\langle M_{*}, x\right\rangle$ is a nondegenerate 4-dimensional orthogonal space, and for each involution $t_{x} \in O^{\varepsilon}(M)$ inducing a transvection on $M_{x}$ with center $M_{*}, t_{x}$ centralizes the stabilizer $K_{*}$ of $M_{*}$ in $\Omega\left(M_{x}\right)$, completing the proof of (3). Then (4) follows from an easy calculation.

Observe that, collecting the results in this section, we have shown:

Theorem 9.17. Theorem 4 holds when $F^{*}(Y)$ is a classical group.

We close this section with two results useful in the proof of Theorem 2.

Lemma 9.18. Assume $G=O^{\varepsilon}(M)$ with $n \geq 6$, and let $u \in c_{2}$. Then $u \in D \leq G$ with $D \cong D_{2\left(q^{2}+1\right)}$ such that $C_{\Gamma O^{\varepsilon}(M)}(D) \cong O_{n-4}^{-\varepsilon}(q)$.

Proof. Let $M=M_{1} \perp M_{2}$ where $M_{1}$ is a nondegenerate 4-dimensional subspace of sign -1 . Then $M_{2}=M_{1}^{\perp}$ is of dimension $n-4$ and $\operatorname{sign}-\varepsilon$, and $K_{1}:=$ $C_{G}\left(M_{2}\right) \cong O\left(M_{1}, Q\right) \cong O_{4}^{-}(q)$ is $L_{2}\left(q^{2}\right)$ extended by a field automorphism. Further $\operatorname{Inv}\left(E\left(K_{1}\right)\right) \subseteq c_{2}$, so we may take $u \in D \leq K_{1}$ with $D \cong D_{2\left(q^{2}+1\right)}$. As $D$ is irreducible on $M_{1}, C_{G}(D)=C_{G}\left(M_{1}\right) \cong O\left(M_{2}, Q\right) \cong O_{n-4}^{-\varepsilon}(q)$. Hence the lemma holds.

Lemma 9.19. Assume $L=\operatorname{SL}(M) \cong L_{n}(2)$ with $n \in\{5,6\}$. Let $G=\operatorname{Aut}(L)$ and $\tau \in G$ a graph automorphism of $L$ such that $C_{L}(\tau) \cong \operatorname{Sp}_{4}(q), \operatorname{Sp}_{6}(q)$, for $n=5,6$, respectively. Then $\tau \in D \leq G$ such that $D \cong D_{2 m}$, and:

(1) If $n=5$, then $m=31$ and $C_{G}(D)=1$.

(2) If $n=6$, then $m=7$ and $C_{G}(D) \cong L_{2}(8)$. 
Proof. Let $E$ be a field extension of $F=\mathbb{F}_{2}$ of degree $r$, where $r=5,3$, for $n=5,6$, respectively. Then $M$ admits the structure $M_{E}$ of an $n / r$-dimensional $E$-space, and the stabilizer $Y$ in $L$ of that structure is isomorphic to $\Gamma L\left(M_{E}\right)$.

Suppose first that $n=5$. Then $Y$ is $X \cong E^{\#} \cong \mathbb{Z}_{31}$ extended by $\operatorname{Aut}(E) \cong \mathbb{Z}_{5}$. Further $X \in \operatorname{Syl}_{31}(L)$ and $Y=N_{L}(X)$, so by a Frattini argument, $G=L N_{G}(X)$. By 19.8 and 19.9 in [ASe], all involutions in $G-L$ are conjugate to $\tau$. Then as $31 \notin \pi\left(C_{L}(\tau)\right)$, some conjugate of $\tau$ inverts $X$, so that (1) holds.

So assume $n=6$. Here $Y_{0}:=O^{2}(Y)=X \times W$, with $X \cong E^{\#} \cong \mathbb{Z}_{7}$ and $W=E(Y) \cong L_{2}(8)$. Further $Y$ is $Y_{0}$ extended by an element $f$ of order 3 inducing a field automorphism on $X$ and $W$. Now $L$ is transitive on such subgroups, and $\operatorname{Aut}(W)=W\langle f\rangle$, so by a Frattini argument, there exists an involution $t$ inverting $X$ and centralizing $W\langle f\rangle$. Finally from 19.8 and 19.9 in [ASe], all involutions in $G-L$ centralizing an $L_{2}(8)$-subgroup of $L$ are conjugate to $\tau$, so (2) holds.

\section{Chev (2)}

In this section we assume the following hypothesis:

Hypothesis 10.1. $G$ is an almost simple finite group with $F^{*}(G)=L$ a group of Lie type $G(q)$ over a field of even order $q$, or $L$ is the Tits group and $q=2$. Let $\Phi$ be a root system for $L$ and $l$ the Lie rank of $L$. Given $\alpha \in \Phi$, let $\hat{U}_{\alpha}$ be the root group of $\alpha, U_{\alpha}=\Omega_{1}\left(\hat{U}_{\alpha}\right), K(\alpha)=\left\langle U_{\alpha}, U_{-\alpha}\right\rangle$, and $X(\alpha)=C_{L}(K(\alpha))$.

Lemma 10.2. Assume $l>1$ and let $\alpha \in \Phi$. Assume either $\alpha$ is a long root, or $L \cong \operatorname{Sp}_{2 l}(q)$ and $\alpha$ is a short root. Set $K=K(\alpha), X=X(\alpha)$, and pick $u \in U_{\alpha}^{\#}$. Then:

(1) Either $K \cong L_{2}(q)$ or $L \cong{ }^{2} F_{4}(q)$ and $K \cong S z(q)$, or $L$ is the Tits group and $K \cong D_{10}$. In any event $H_{K}=N_{K}\left(U_{\alpha}\right) \cap N_{K}\left(U_{-\alpha}\right) \cong \mathbb{Z}_{q-1}$ is a Cartan subgroup of the Borel subgroup $N_{K}\left(U_{\alpha}\right)$ of $K$.

(2) $P=P(\alpha)=N_{L}\left(U_{\alpha}\right)$ is a parabolic subgroup of $L$, and if $L$ is not $L_{n}(q)$ then $P$ is a maximal parabolic.

(3) $X \times H_{K}=P \cap P(-\alpha)$ is a Levi factor of $P$.

(4) $C_{L}(u)=R(P) X$ and $\left(K, U_{\alpha}\right) \in \mathfrak{S}(L, u)$.

(5) Assume $G=L V$ for some elementary abelian 2-group $V$. Then either

(i) $\mathrm{O}_{2}\left(C_{G}(K)\right)=1$, or

(ii) $L \cong L_{3}(q)$ with $q \leq 4$, or $L_{4}(2)$, and $O_{2}\left(C_{G}(K)\right)=\langle\tau\rangle$, where $\tau$ induces a graph automorphism on $L$. 
Proof. Parts (1) and (2) are well known; for instance see Example 3.2.6 in [GLS3].

Let $P^{\prime}=P(-\alpha)$. We claim that $P$ and $P^{\prime}$ are opposite parabolics. Namely conjugating in the Weyl group of $L$, we may assume $\alpha$ is the root of highest height, or $L \cong \operatorname{Sp}_{2 l}(q)$ and $\alpha$ is the short root of highest height. Then, in the notation of Section 47 of [A4], and in particular in the notation before 47.4 in [A4], $P=P_{J}$ for some subset $J$ of the set $\pi$ of simple roots for some ordering of $\Phi, R(P)=$ $V_{J}=\left\langle U_{\gamma}: \gamma \in \psi_{J}\right\rangle$, and $L_{J}=\left\langle U_{\beta}, U_{-\beta}: \beta \in \psi_{J}\right\rangle$. Then $P^{\prime}=V_{-J} L_{-J}$ and $L_{-J}=L_{J}$, while $V_{J} \cap V_{-J}=1$, so using the Bruhat decomposition (cf. 47.2 in $[\mathrm{A} 4]$ )

$$
\begin{aligned}
P \cap P^{\prime} & =L_{J} H_{K} V_{J} \cap L_{J} H_{K} V_{-J}=L_{J} H_{K}\left(L_{J} H_{K} V_{J} \cap V_{-J}\right) \\
& =L_{J} H_{K}\left(V_{J} \cap V_{-J}\right)=L_{J} H_{K}=Y,
\end{aligned}
$$

where $Y:=L_{J} H_{K}$ is a Levi factor of $P$ and $P^{\prime}$. This establishes the claim.

As $U=U_{\alpha}$ and $U^{\prime}=U_{-\alpha}$ are normal in $P$ and $P^{\prime}$, respectively, $Y$ acts on $K=\left\langle U, U^{\prime}\right\rangle$. Further $H_{K} \leq Y$ and $H_{K} \cong \operatorname{Aut}_{L}(U)$, so $Y=H_{K} C_{Y}(U)$. Then as $C_{\text {Aut }(K)}(U) \cap N_{\text {Aut }(K)}\left(U^{\prime}\right)=1, C_{Y}(U)=C_{Y}(K)=Y \cap X$. Thus $Y=H_{K}(X \cap Y)$. On the other hand $X \leq N_{L}(U) \cap N_{L}\left(U^{\prime}\right)=P \cap P^{\prime}=Y$, so $X=X \cap Y$, completing the proof of (3).

By choice of $\alpha$ and the Chevalley commutator relations, $U$ is in the center of the Sylow 2-subgroup $S=\left\langle U_{\beta}: \beta \in \Phi^{+}\right\rangle$of $L$. Thus (cf. 47.7 in [A4]) setting $L_{u}:=$ $O^{2^{\prime}}\left(C_{L}(u)\right), L_{u} H$ is a parabolic subgroup of $L$. By construction, $P=R(P) X H_{K} \leq$ $L_{u} H$, so if $P$ is maximal then $P=L_{u} H$ and $L_{u}=C_{P}(u)=R(P) X$. If $P$ is not maximal, then $L \cong L_{n}(q)$ by (2), where it is well known that $U=R t_{L}(u)$ so that $P=L_{u} H$ and $L_{u} R(P) X$. Therefore $U \leq Z\left(C_{L}(u)\right.$ ), so (4) follows from (3).

We next prove (5), so assume $G=L V$ for some elementary abelian 2-group $V$. We adopt the terminology in 2.5.13 of [GLS3] when discussing involutory automorphisms of $L$. As $O_{2}(X)=1, O_{2}\left(C_{L}(K)\right)=1$ by (4), so we may assume $V \not \subset L$. If $G$ contains a field or graph-field automorphism (excluding $L$ of type $B_{2}$ or $F_{4}$ ), then some involutory field or graph-field automorphism $\sigma$ acts as a field automorphism on $K$, and hence $C_{L\langle\sigma\rangle}(K)=X$. Therefore (5) (i) holds unless $G$ contains a graph automorphism $\tau$ (or graph-field in the case of $B_{2}$ and $F_{4}$ ). Therefore we may assume $L$ is $L_{n}^{\varepsilon}(q), \operatorname{Sp}_{4}(q), D_{m}^{\varepsilon}(q), m \geq 4, F_{4}(q)$, or $E_{6}^{\varepsilon}(q)$. If $L$ is $\operatorname{Sp}_{4}(q)$ or $F_{4}(q)$ then $N_{L\langle\tau\rangle}(K) \leq L$, and hence (5) (i) holds. Thus we may assume we are in one of the remaining cases, where some involutory graph automorphism $\tau$ centralizes $K$.

Suppose $L \cong L_{3}^{\varepsilon}(q)$. As $l>1, \varepsilon=+1$. Then $K=C_{L}(\tau)$, so $\tau$ inverts $X$ and (5) (i) holds unless $X=1$. In that event $q \leq 4$ and (5) (ii) holds.

In the remaining cases, $O^{2^{\prime}}(X)=M$ is of Lie type over $\mathbb{F}_{q}$, and described in 12.1 in [ASe]. Further $\tau$ induces a graph automorphism on $M$, so $\tau$ induces an outer automorphism on $M$ unless $L \cong L_{4}^{\varepsilon}(q)$, where $M \in K^{L}$ is centralized by $\tau$. In the first case (5) (i) holds, so we may assume the latter. Then from 19.9 in [ASe], $C_{L}(\tau) \cong \mathrm{Sp}_{4}(q)$, so $M=C_{X}(\tau)$. Therefore $\tau$ inverts $O(X)$, so (5) (i) holds unless 
$O(X)=1$. As $O(X)$ is of order $q-\varepsilon$, we conclude that (5) (ii) holds when $O(X)=1$, completing the proof of (5).

Lemma 10.3. Suppose $L$ is $S z(q)$, let $u$ be an involution in $L$, and $U=Z(R(B))$ where $B$ is a Borel subgroup of $L$ containing $u$. Then $(L, U) \in \mathfrak{S}(L, u)$.

Proof. This is essentially immediate from the definitions.

Lemma 10.4. Assume $L$ is exceptional and either $l=2$ or $L$ is the Tits group. Then:

(1) $L \cong G_{2}(q),{ }^{3} D_{4}(q),{ }^{2} F_{4}(q)$, or the Tits group.

(2) L has two classes of involutions; the long and short root involutions.

(3) Let $u \in U_{\alpha}$ be a short root involution. If $L$ is not ${ }^{3} D_{4}(q)$ set $K=K(\alpha)$ and $U=U_{\alpha}$. If $L$ is ${ }^{3} D_{4}(q)$ let $U=\left\{U_{\alpha}(a): a \in \mathbb{F}_{q}\right\}$ and $K=\left\langle U, s_{\alpha}\right\rangle$, where $s_{\alpha}$ is the reflection through $\alpha$. Then $(K, U) \in \mathbb{S}(L, u)$.

(4) Let $G=L V$ for some elementary abelian 2-group $V$. Then $O_{2}\left(C_{G}(K)\right)=1$.

Proof. Part (1) follows by inspection of the list of groups of Lie type, and (2) appears in Section 18 of [ASe].

The proof of (3) is similar to that of parts (3) and (4) of 10.2. First choose $\alpha$ to be the short root of highest height. From Section 18 of [ASe], $C_{L}(u) \leq P=P_{J}$ is a maximal parabolic of $L$ such that $P=R(P) H_{0} L_{J}$, where $L_{J} \cong L_{2}(q)$ centralizes $U$, and $H_{0}$ is a Cartan subgroup of $K(\alpha)$. Then $L_{J}=\left\langle U_{\beta}, U_{-\beta}\right\rangle$ centralizes $K(\alpha)=$ $\left\langle U_{\alpha}, U_{-\alpha}\right\rangle$. On the other hand as in the proof of 10.2(3), $P$ and $P^{\prime}=P_{-J}$ are opposite parabolics, so $C_{L}(K) \leq P \cap P^{\prime}=L_{J} H_{0}$, and hence $C_{L}(K)=L_{J} C_{H_{0}}(U)=L_{J}$. This establishes (3).

Assume the hypothesis of (4). By (3) we may assume $|\operatorname{Out}(L)|$ is even, so $L$ is not ${ }^{2} F_{4}(q)$. Similarly (4) holds for the Tits group, as its automorphism group is ${ }^{2} F_{4}(2)$. Finally if $L$ is $G_{2}(q)$ or ${ }^{3} D_{4}(q)$ and $|\operatorname{Out}(L)|$ is even then from 19.1 in [ASe], $|G: L|=2$ and each involution in $G-L$ is a field automorphism. In particular some field automorphism of order 2 induces a field automorphism of order 2 on $K$, so (4) holds.

During much of the remainder of the paper we will assume the following hypothesis:

Hypothesis 10.5. Hypothesis 10.1 holds with $L$ an exceptional group and $l \geq 4$; that is $L$ is $F_{4}(q), E_{6}^{\varepsilon}(q), E_{7}(q)$, or $E_{8}(q)$. Adopt the notation on page 5 of [ASe] for labeling the simple roots $\alpha_{i}, i \in I=\{1, \ldots, l\}$ in $\Phi$, and adopt the notation on page 4 of [ASe] for labeling parabolics $P_{S}, S \subseteq I$. In particular $Q_{S}=R\left(P_{S}\right)$ is the radical of $P_{S}$, and $L_{S}=O^{2^{\prime}}\left(\bar{L}_{S}\right)$, where $\bar{L}_{S}$ is a Levi factor of $P_{S}$. Let $\xi$ be the root of highest height and let $U=U_{\xi}, K=K(\xi)$, and $X=X(\xi)$. Let $H_{K}$ be the Cartan subgroup of $K$ defined in 10.2(1). 
Lemma 10.6. Assume $\alpha \in \Phi$ is a long root and let $U=U_{\alpha}, K=K(\alpha)$, and $X=X(\alpha)$. Let $u \in U^{\#}$ and $t \in X$ be involutions, and $t \in K_{t} \leq X$ with $K \cong K_{t}$. Set $v=u t$, let $K_{v}$ be the full diagonal subgroup of $K \times K_{t}$, and $Y=C_{X}\left(K_{t}\right)$. Let $P$ be a parabolic subgroup of $L$ such that $C_{L}(v) \leq P$, set $R=R(P)$, and assume that

(1) $O^{2^{\prime}}\left(C_{P}(v) / O_{2}\left(C_{R}(v)\right)\right)$ and $O^{2^{\prime}}(Y)$ are reductive groups of Lie type and even characteristic of the same Lie rank.

(2) $U \leq Z\left(C_{R(P)}(v)\right)$.

Then $O_{2}\left(C_{L}\left(K_{v}\right)\right)=1$.

Proof. By definition, $X=C_{L}(K)$, so as $K_{v}$ is a full diagonal subgroup of $K \times K_{t}$ and $K_{t} \leq X$, we have $C_{X}\left(K_{v}\right)=C_{X}\left(K_{t}\right)=Y$. In particular $Y \leq H=C_{L}\left(K_{v}\right)$. Suppose $Q=O_{2}(H) \neq 1$. Then $U Y Q \leq C_{L}(v)=C_{P}(v)$. Set $M=O^{2^{\prime}}\left(C_{P}(v)\right)$, $M^{*}=M / C_{R}(v)$ and $Y_{0}=O^{2^{\prime}}(Y)$. By (1), $Y_{0}$ is reductive, so $O_{2}\left(Y_{0}\right)=1$ and hence $Y_{0}^{*} \cong Y_{0}$. By (1), $M^{*}$ is reductive so $O_{2}\left(M^{*}\right)=1$ and hence $O_{2}\left(C_{L}(v)\right)=$ $C_{R}(v)$. Further $M^{*}$ and $Y_{0}^{*}$ have the same Lie rank by (1), so as $Q^{*}$ is a $Y$-invariant 2-subgroup of $M^{*}$, it follows from 7.4 that $Q^{*}=1$. Thus $Q \leq C_{R}(v)$, so by (2), $u$ centralizes $Q$. Then as $C_{L}(u)=C_{L}(U), U$ centralizes $Q$, so $K=\left\langle U^{K_{v}}\right\rangle$ centralizes $Q$. Therefore $Q \leq C_{L}(K)=X$, so $Q \leq O_{2}\left(C_{X}\left(K_{v}\right)\right)=O_{2}(Y)$, contradicting $Y$ reductive.

\section{1. $E_{6}^{\varepsilon}(q)$}

In this section we assume Hypothesis 10.5 with $L=E_{6}^{\varepsilon}(q)$ and adopt the following notation.

Notation 11.1. Let $F=\mathbb{F}_{q}, \mathbb{F}_{q^{2}}$, for $\varepsilon=+1,-1$, respectively. Let $\hat{L}$ be the universal group of type $E_{6}(F)$ and $M$ a faithful 27-dimensional $F \hat{L}$-module. The module $M$ is described in detail in [A5]. Let $\hat{G}$ be $\hat{L}$ extended by field automorphisms, so that $\widehat{G} \leq \Gamma L(M)$. Regard $L$ as the image of the subgroup $\hat{L}_{\sigma}$ of fixed points on $\hat{L}$ of $\sigma \in \operatorname{Aut}(\hat{L})$ under the projective map, where $\sigma=1$ if $\varepsilon=+1$ and $\sigma$ is a graph-field automorphism if $\varepsilon=-1$. Let $\widehat{K}$ be a fundamental subgroup of $\hat{L}$ and $\hat{X}=C_{\hat{L}}(\widehat{K})$. Then $K, X$ are the image of $\hat{K}_{\sigma}, \widehat{X}_{\sigma}$ in $L$, respectively.

Lemma 11.2. (1) $P(\xi)=P_{r}$, where $r=2,1$ for $\varepsilon=+1,-1$, respectively. Thus $L_{1}=X \cong \mathrm{SL}_{6}^{\varepsilon}(q)$.

(2) $Q_{r} \cong q^{1+20}$ is special with center $U$.

(3) If $\varepsilon=+1$, then $L_{6} \cong \Omega_{10}^{+}(q)$ and $Q_{6} \cong E_{q 16}$ is a spin module for $L_{6}$. Further $Q_{2} \cap Q_{6}$ is of rank 11 , and $L_{2,6} \cong \mathrm{SL}_{5}(q)$ acts naturally on $Q_{6} /\left(Q_{2} \cap Q_{6}\right)$. 
(4) If $\varepsilon=-1$, then $L_{4} \cong \Omega_{8}^{-}(q), Q_{4} \cong q^{8+16}$ is special, $Z\left(Q_{4}\right)$ is the natural module for $L_{4}$, and $Q_{4} / Z\left(Q_{4}\right)$ is a spin module.

Proof. From the discussion in Sections 14 and 15 in [ASe], $P_{r}=P(\xi), Q_{r} \cong q^{1+20}$, and $L_{r} \cong L_{6}^{\varepsilon}(q)$. Then $L_{1}=X$ by 10.2 (3) establishing (1) and (2). Part (4) follows from 4.6 in [CKS], and the first statement in (3) appears in 3.14 in [A5]. The second follows as the radical of $P_{2,6} \cap X$ is the natural module for $L_{2,6} \cong \mathrm{SL}_{5}(q)$, and $Q_{2,6}=Q_{2} Q_{6}$.

Lemma 11.3. (1) L has three classes of involutions with representatives $u_{i}, 1 \leq i \leq 3$, denoted by $x, y, z$ in 15.1 of [ASe] when $\varepsilon=+1$, and $t, u$, $v$ in 14.1 of [ASe] when $\varepsilon=-1$, respectively. Further $u_{1} \in U$.

(2) Let $m_{i}=\operatorname{dim}\left(\left[M, u_{i}\right]\right)$. Then $m_{1}=6, m_{2}=10$, and $m_{3}=12$.

(3) The involutions in $X$ are of type $j_{i}, 1 \leq i \leq 3$.

(4) $u_{i}$ is $L$-conjugate to a member of $j_{i}$ for $1 \leq i \leq 3$.

(5) Let $J_{i}, 1 \leq i \leq 3$, be commuting fundamental subgroups of $X, J_{4}=K$, and $\Delta=\left\{J_{i}: 1 \leq i \leq 4\right\}$. Then $N_{L}(\Delta)$ induces $\operatorname{Sym}(\Delta)$ on $\Delta$.

(6) Let $N_{6}$ be the natural module for $X$ and for $i$ an involution in $X$ and $g \in L$, set $R t_{L}\left(i^{g}\right)=R t_{\mathrm{GL}\left(N_{6}\right)}(i)^{g}$. Then $R t_{L}\left(i^{g}\right)$ is well defined and $R t_{L}\left(i^{g}\right) \leq Z\left(C_{L}\left(i^{g}\right)\right)$.

Proof. Part (1) is 14.1 and 15.1 in [ASe], while (3) follows from 11.1 (1), 9.2, and 9.8.

Define $\Delta$ as in (5), and let $X_{i}=C_{L}\left(J_{i}\right)$. Then $\Delta=\left\{J_{i}\right\} \cup\left(\Delta \cap X_{i}\right)$, and $N_{X_{i}}(\Delta)$ induces the symmetric group $S_{3}$ on $\Delta-\left\{J_{i}\right\}$, so (5) follows.

Assume for the moment that $\varepsilon=+1$. We abuse notation and write $K, X$ for $\hat{K}, \hat{X}$. As observed in Section 4 of [A6], the members $i$ of $j_{1}$ are the root involutions in $X$, and $R t_{L}(i)$ is the root group of $i$ in $L$. Then (6) holds for the involutions $i \in j_{m}$ for $m=2,3$ by 4.4 and 4.5 in [A6]. From page 167 in [A5], [K, $M$ ] is the sum of six copies of the natural module for $K$, so $m([M, i])=6$ for $i$ an involution in $K$, and hence $m_{1}=6$ by (5). Also $m_{2}=10$ and $m_{3}=12$ by 4.1 and 4.2 in [A6]. Thus (2) holds in this case. From 4.3 in [A6], each involution in $L$ is conjugate to an involution in $j_{m}$ for a unique $m$. Following Section 4 in [A6], call such involutions involutions of type $m$. The isomorphism type of the centralizers of involutions of type 2 appears in 4.6 of [A6], and comparing this to 15.5 in [ASe], the involution $y$ in 15.1 of [ASe] is of type 2. Then as involutions of type 1 and the involution $x$ in 15.1 of [ASe] are root involutions, it follows that the involution $z$ in 15.1 of [ASe] is of type 3 . Thus (4) holds in this case.

We have established the lemma when $\varepsilon=+1$. When $\varepsilon=-1$, we may choose $\sigma$ to act on $\hat{K}, \hat{X}$, and $\hat{K}_{i}$, and then to centralize $u_{m} \in j_{m}$. Then $u_{m} \in \hat{L}_{\sigma}$ is in the class $j_{m}$ in $X=\hat{X}_{\sigma} \cong \mathrm{SU}_{6}(q)$, so (2) and (4) hold when $\varepsilon=-1$, modulo verifying the correspondence between the $u_{m}$ and $t, u, v$ in (1). As $\operatorname{dim}\left(\left[u_{m}, M\right]\right) \neq$ $\operatorname{dim}\left(\left[u_{k}, M\right]\right)$ for $m \neq k, u_{m}, 1 \leq m \leq 3$ are representatives for the three classes of 
involutions in $L$. The graph-field automorphism $\sigma$ induces a field automorphism on $C_{\hat{L}}\left(u_{2}\right) / O_{\infty}\left(C_{\hat{L}}\left(u_{2}\right)\right) \cong \operatorname{Sp}_{6}\left(q^{2}\right)$, so $C_{L}\left(u_{2}\right) / O_{\infty}\left(C_{L}\left(u_{2}\right)\right) \cong \operatorname{Sp}_{6}(q)$, and hence $u_{2}$ is conjugate to the involution $u$ in 14.3 of [ASe]. This verifies the correspondence between our two labelings of involutions, and completes the proof of (1). Part (6) follows as $R t_{L}(i)=R t_{\hat{L}}(i)_{\sigma}$.

Lemma 11.4. For $1 \leq m \leq 3$, let $u_{m} \in j_{m}$ and $U_{m}=R t_{L}\left(u_{m}\right)$. Then:

(1) There exist $K_{m} \leq X$ with $\left(K_{m}, U_{m}\right) \in \mathfrak{S}\left(X, u_{m}\right)$.

(2) $C_{L}\left(u_{m}\right)=C_{L}\left(K_{m}\right) O_{2}\left(C_{L}\left(u_{m}\right)\right)$, so if $O_{2}\left(C_{K}\left(u_{m}\right)\right)=1$, then $\left(K_{m}, U_{m}\right) \in$ $\mathfrak{S}\left(L, u_{m}\right)$.

(3) $\mathrm{O}_{2}\left(C_{G}\left(K_{m}\right)\right) \leq L$.

Proof. Part (1) was established in 9.16. In particular $K_{m} \cong L_{2}(q)$ and $u_{m} \in U_{m} \cong$ $E_{q}$. By 11.3(6), $U_{m} \leq Z\left(C_{L}\left(u_{m}\right)\right)$. Thus to establish (2), it remains to show that $C_{L}\left(K_{m}\right)$ is a supplement to $O_{2}\left(C_{L}\left(u_{m}\right)\right)$ in $C_{L}\left(u_{m}\right)$. By 10.2(4), we may assume $m=2$ or 3 .

First take $m=3$. Then the discussion in Section 9 (or 4.3 and 6.2 in [ASe]) says that $C_{X}\left(K_{3}\right) \cong L_{3}^{\varepsilon}(q)$. Set $B_{3}:=K C_{X}\left(K_{3}\right)$. Then $B_{3} \cong L_{2}(q) \times L_{3}^{\varepsilon}(q)$, and by 14.3 and 15.5 in [ASe], $B_{3}$ is a complement to $O_{2}\left(C_{L}\left(u_{3}\right)\right)$ in $C_{L}\left(u_{3}\right)$, so (2) holds when $m=3$.

Next suppose that $m=2$. Then from the discussion in Section 9 (or 4.3 and 6.2 in [ASe] $), C_{X}\left(K_{2}\right) \cong \mathrm{GL}_{2}(q) \times L_{2}(q)$, so $B_{2}:=K C_{X}\left(K_{2}\right) \cong \mathrm{GL}_{2}(q) \times L_{2}(q)^{2}$. Let $Y=O^{2^{\prime}}\left(C_{L}\left(u_{2}\right)\right)$ and $Y^{*}=Y / O_{2}(Y)$. By 14.3 and 15.5 in [ASe], $Y^{*} \cong \operatorname{Sp}_{6}(q)$. Let $\Delta=\left\{J_{i}: 1 \leq i \leq 4\right\}$ be as in 11.3 (5). From the discussion in Section 9, we may take $K_{2}$ to be a full diagonal subgroup of $J_{1} J_{2}$. Then by 11.3(5), there is $g \in N_{L}(\Delta)$ with $K_{2}^{g}$ a full diagonal subgroup of $J_{1} J_{4}=K J_{1}$. Let $Y_{0}^{g}=C_{X}\left(K_{2}^{g}\right)$. Then $Y_{0}^{g}=C_{X}\left(J_{1}\right) \cong \mathrm{GL}_{4}^{\varepsilon}(q)$ from Section 9 . Hence $Y_{0}^{*} \cong \Omega_{6}^{\varepsilon}(q)$, and $N_{Y^{*}}\left(Y_{0}^{*}\right) \cong$ $O_{6}^{\varepsilon}(q)$ is the unique maximal overgroup of $Y_{0}^{*}$ in $Y^{*}$. Also $B_{0}=O^{2}\left(O^{2^{\prime}}\left(B_{2}\right)\right) \not \leq$ $Y_{0} O_{2}\left(C_{L}\left(u_{2}\right)\right)$ as $B_{0}^{g}$ does not centralize $K J_{1}$. Therefore $C_{L}\left(K_{2}\right)^{*}=Y^{*}$, and hence $C_{L}\left(K_{2}\right)$ is a supplement to $O_{2}\left(C_{L}\left(u_{2}\right)\right)$ in $C_{L}\left(u_{2}\right)$, completing the proof of (2).

Suppose $\varepsilon=+1$. Then $\operatorname{Aut}(L)$ is $L$ extended by $\Lambda \times\langle\tau\rangle$, where $\Lambda$ is a group of field automorphisms and $\tau$ is a graph automorphism. Further we may choose $\Lambda$ to induce a group of field automorphisms on $K_{m}$, and from 19.7 in [ASe], $C_{L}(\tau) \cong F_{4}(q)$ and we may choose $\tau$ to centralize $K$ and induce a graph automorphism on $X$ with $J_{i} \leq C_{X}(\tau) \cong \operatorname{Sp}_{6}(q)$ for each $J_{i} \in \Delta$. Then from 9.5, $\tau$ centralizes $K_{m}$ and induces a graph automorphism on $C_{X}\left(K_{m}\right)$, and on $Y_{0}$ when $m=2$. Thus (3) holds in this case. On the other hand when $\varepsilon=-1, L$ is the image of $\hat{L}_{\sigma}$ and $\sigma=\tau \lambda$, where $\lambda$ induces an involutory field automorphism on $\hat{L}$. Then $\operatorname{Out}(L)$ is cyclic with $\tau$ inducing the involutory graph automorphism on $L$, so from the discussion above, $\tau$ is faithful on $C_{L}\left(K_{m}\right)$, and hence (3) holds again. 
Lemma 11.5. $\left(K_{2}, U_{2}\right) \in \mathfrak{S}\left(L, u_{2}\right)$ and $O_{2}\left(C_{G}\left(K_{2}\right)\right)=1$.

Proof. By 11.4 it suffices to show that $O_{2}\left(C_{L}\left(u_{2}\right)\right)=1$.

As we saw during the proof of $11.4, K_{2}^{g} \leq K J_{1}$ for some $g \in N_{L}(\Delta)$, so $v_{2}:=u_{2}^{g}=w_{1} w_{4}$ for some $w_{i} \in J_{i}$. Suppose for the moment that $\varepsilon=+1$. Choose notation so that $w_{1} \in V=R\left(P_{2,6}\right) \cap X$. From 11.2(3), $V$ is a complement to $Q_{2} \cap Q_{6}$ in $Q_{6}$. Thus $v_{2} \in Q_{6}$, and from the proof of 11.4, $C_{X}\left(K_{2}^{g}\right)=C_{X}\left(J_{1}\right) \cong L_{4}(q)$. From 15.4 and 15.5 in [ASe], $C_{L}\left(v_{2}\right) \leq P \in P_{6}^{L}$, and $O^{2^{\prime}}\left(C_{L}\left(v_{2}\right)\right) / O_{2}\left(C_{L}\left(v_{2}\right)\right) \cong$ $\operatorname{Sp}_{6}(q)$. Then as $C_{X}\left(J_{1}\right)$ and $\operatorname{Sp}_{6}(q)$ both are of Lie rank 3, and $C_{X}\left(J_{1}\right)$ acts on $Q_{6} \leq C_{L}\left(v_{2}\right)$, it follows from 7.4 that $Q_{6} \leq O_{2}\left(C_{L}\left(v_{2}\right)\right)$. Then as $Q_{6}$ is weakly closed in $P_{6}$ with respect to $L, C_{L}\left(v_{2}\right) \leq P_{6}$. Now 10.6 completes the proof in this case.

Finally suppose $\varepsilon=-1$. Then $\sigma$ induces a field automorphism on $\hat{K}_{2}$ and $O^{2^{\prime}}\left(C_{\hat{L}}\left(\widehat{K}_{2}\right)\right)$, so

$$
O^{2^{\prime}}\left(C_{L}\left(K_{2}\right)\right)=O^{2^{\prime}}\left(C_{\hat{L}}\left(\hat{K}_{2}\right)_{\sigma}\right) \cong \operatorname{Sp}_{6}(q)
$$

as $\widehat{K}_{2}=\left\langle K_{2}, \hat{U}_{2}\right\rangle$ and $\hat{U}_{2} \leq Z\left(C_{\hat{L}}\left(u_{2}\right)\right)$.

Lemma 11.6. Let $s=4,2$ for $\varepsilon=+1,-1$, respectively, $g \in P_{s}-P_{r}, I=U U^{g}$, $E=Q_{r} \cap Q_{r}^{g}$, and $Y:=\left\langle Q_{r}, Q_{r}^{g}\right\rangle$. Then:

(1) $Q_{s}=\left(Q_{r} \cap Q_{s}\right)\left(Q_{r}^{g} \cap Q_{s}\right)$ and $Y / Q_{s} \cong L_{2}(q)$.

(2) $L_{s}=\left(Y \cap L_{s}\right) \times Y_{r, s}$ with $Y_{s}:=Y \cap L_{s} \cong L_{2}(q)$ and $L_{r, s} \cong \operatorname{SL}_{3}(q) \times \operatorname{SL}_{3}(q)$ or $\mathrm{SL}_{3}\left(q^{2}\right)$ for $\varepsilon=+1,-1$, respectively.

(3) $I=Z\left(Q_{s}\right)$ is the natural module for $Y_{s}$.

(4) $[Y, E]=I$ and $E / I$ is a tensor product module of order $q^{9}$ for $L_{r, s}$.

(5) $Q_{s} / E$ is the tensor product of the natural module for $Y_{S}$ and the dual of $E / I$ for $L_{1,6}$.

(6) There exists $v_{3} \in u_{3}^{L}$ with $v_{3} \in E-I$, such that $C_{Q_{s}}\left(v_{3}\right)=O_{2}\left(C_{L}\left(v_{3}\right)\right)$, $C_{L_{s}}\left(v_{3}\right)=Y_{s} \times C_{L_{r, s}}\left(v_{3}\right)$, and $C_{L_{r, s}}\left(u_{3}\right) \cong \operatorname{SL}_{3}^{\varepsilon}(q)$.

(7) $\left(K_{3}, U_{3}\right) \in \mathcal{S}\left(L, u_{3}\right)$ and $O_{2}\left(C_{G}\left(K_{3}\right)\right)=1$.

Proof. Parts (1)-(5) follow from the standard theory of large special groups; see for example 8.15 in [A8]. From 15.4 and 14.2 in [ASe], there is $v_{3} \in u_{3}^{L}$ such that $C_{L}\left(v_{3}\right) \leq P_{s}$ with $O_{2}\left(C_{L}\left(v_{3}\right)\right)=C_{Q_{s}}\left(v_{3}\right)$, and $O^{2^{\prime}}\left(C_{L}\left(v_{3}\right)\right) / O_{2}\left(C_{L}\left(v_{3}\right)\right)$ as described in (6). From the action of $L_{s}$ on $Q_{s}$ described in (1)-(5), it follows that $v_{3} \in E$.

Suppose for the moment that $\varepsilon=+1$ and consider the parabolic $X_{4}=X \cap P_{4}$ of $X$. Then $W=R\left(X_{4}\right) \cong E_{q^{9}}$ is the tensor product module for $L_{2,4} \cong \mathrm{SL}_{3}(q) \times$ $\mathrm{SL}_{3}(q)$. From 11.3(4), we may pick $u_{3} \in W \cap j_{3}$, and from Section 4 in [ASe], 
$B_{4}:=C_{L_{2,4}}\left(u_{3}\right) \cong \mathrm{SL}_{3}(q)$ and $W$ is the adjoint module for $B_{4}$. Then $K B_{4}$ is contained in a Levi factor $L_{2,4}^{\prime}$ in $P_{4}^{\prime} \in P_{4}^{L}$ with $C_{L}\left(u_{3}\right) \leq P_{4}^{\prime}$, completing the proof of (6) in this case.

Further $K_{2}=O^{2^{\prime}}\left(C_{X}\left(B_{4}\right)\right), O^{2^{\prime}}\left(C_{X}\left(K_{3}\right)\right)=B_{4}$, and $K_{3}$ centralizes $K B_{4}$. Suppose $O_{2}\left(C_{L}\left(K_{3}\right)\right)=R \neq 1$. Then as $R$ is normalized by $K B_{4}, R \leq O_{2}\left(C_{L}\left(u_{3}\right)\right)$. If $1 \neq I^{\prime} \cap R$, then as $K$ is irreducible on $I^{\prime}, I^{\prime} \leq R$. But then $K_{3} \leq C_{L}\left(I^{\prime}\right) \leq P^{\prime}$, contradicting $u_{3} \in Q_{4}^{\prime}$. Hence $I^{\prime} \cap R=1$. Similarly $C_{W}\left(K_{3}\right)=1$ and $W$ and $I^{\prime}$ contain all proper $K B_{4}$-submodules of $E^{\prime}$, so $E^{\prime} \cap R=1$. Then as $K B_{4}$ is irreducible on $Q^{\prime \prime}=C_{Q_{4}^{\prime}}\left(u_{3}\right) / E^{\prime}$, it follows that $Q^{\prime \prime}=C_{Q_{4}^{\prime}}\left(K_{3}\right) E$, so $Q^{\prime \prime} \leq C_{L}\left(K_{3}\right)$ as $E^{\prime}=\Phi\left(Q^{\prime \prime}\right)$. This contradiction shows that $O_{2}\left(C_{L}\left(K_{3}\right)\right)=1$, and then (7) follows from 11.4.

Finally suppose $\varepsilon=-1$. Then taking fixed points of $\sigma$ on $\hat{L}$, we conclude (6) and (7) also hold in this case.

\section{2. $F_{4}(q)$}

In this section we assume Hypothesis 10.5 with $L=F_{4}(q)$, and adopt the following notation.

Notation 12.1. As in 11.1, let $F=\mathbb{F}_{q}, \hat{L}$ be the universal group of type $E_{6}(F)$, and $M$ a faithful 27-dimensional $F \hat{L}$-module. We regard $L$ as the image of the fixed points $\hat{L}_{\tau} \cong Z(\hat{L}) \times F_{4}(q)$ of a graph automorphism $\tau$ of $\hat{L}$. Let $\hat{K}$ be a fundamental subgroup of $\hat{L}, \hat{X}=C_{\hat{L}}(\hat{K})$, and define $\hat{\Delta}=\{\hat{J}: 1 \leq i \leq 4\}$ as in 11.3 (5). Choose notation so that $\tau$ centralizes $\hat{\Delta}$. Then $\tau$ induces a graph automorphism on $\hat{X}$ and $K$, $J_{i}, X, \Delta$ are the images of $\hat{K}, \hat{J}_{i}, \hat{X}_{\tau}, \hat{\Delta}$ in $L$, respectively. Recall from $11.3(6)$ that $N_{6}$ is the natural module for $\hat{X}=\operatorname{SL}\left(N_{6}\right)$, and observe $X=\operatorname{Sp}\left(N_{6}\right)$.

Lemma 12.2. (1) $P(\xi)=P_{1}$, so $L_{1}=X \cong \operatorname{Sp}_{6}(q)$.

(2) $Q_{1}=E J$, where $E \cong E_{q^{7}}$ is the orthogonal module for $X \cong S_{7}(q)$, $J \cong q^{1+8}$ is special with $[J, E]=1$ and $Z(J)=U=C_{E}(X)$, and $Q_{1} / E$ is the spin module for $X$.

Proof. From the discussion after 13.1 in [ASe], $P_{1}=P(\xi)$ and $L_{1} \cong \operatorname{Sp}_{6}(q)$. Then $L_{1}=X$ by 10.2 (3), establishing (1). Part (2) follows from 4.5 in [CKS].

Lemma 12.3. (1) L has four classes of involutions with representatives $u_{l}, u_{s}, u_{c}$, $v$, denoted by $t, u, t u, v$ in 13.1 of [ASe], respectively. Further $u_{l} \in U$ and $u_{s}$ are long and short root involutions, respectively.

(2) The involutions in $X$ are of type $b_{1}, a_{2}, c_{2}$, and $b_{3}$.

(3) The L-conjugates of $u_{l}, u_{s}, u_{c}, v$ in $X$, are in $b_{1}, a_{2}, c_{2}, b_{3}$, respectively. 
(4) $N_{L}(\Delta)$ induces $\operatorname{Sym}(\Delta)$ on $\Delta$.

(5) Let $r=r_{1} \ldots r_{4}$ with $r_{i} \in J_{i}$ and $r_{i}^{2}=1$. Set $\omega(r)=\left|\left\{i: r_{i} \neq 1\right\}\right|$. Then $r$ is conjugate in $L$ to $u_{l}, u_{c}, v$ for $\omega(r)=1,2,3$, respectively.

Proof. The first part of (1) is 13.1 in [ASe], while (2) is 9.10. Moreover $b_{1}, a_{2}$ are the long, short root involutions of $X$, and from Table 1 on page 5 of [ASe], these root involutions are also long, short root involutions in $L$, in the respective case. This completes the proof of (1).

The proof of (4) is the same as that of $11.3(5)$.

Let $X_{i}:=C_{X}\left(J_{i}\right), U_{i}$ a root group in $J_{i}, V=R\left(C_{X}\left(U_{1}\right)\right)$ and $V^{\prime}=R\left(C_{X_{1}}(U)\right)$, and $Y=C_{X}\left(J_{1}\right)$. Then $N_{J_{1}}\left(U_{1}\right) V Y$ and $H_{K} V^{\prime} Y$ are parabolics in $X$ and $X_{1}$, respectively. Set $P_{1}^{*}=P_{1} / Q_{1}$. From $12.2(2), V^{\prime \prime}=C_{Q_{1}}\left(J_{1}\right)=C_{E}\left(J_{1}\right) \cong E_{q^{5}}$. On the other hand the Lie rank of $J_{1} Y$ and $L_{1}$ is 3 , so $\left(V^{\prime}\right)^{*}=1$ by 7.4. Thus $V^{\prime} \leq V^{\prime \prime}$ and hence $V^{\prime}=V^{\prime \prime}$ as $\left|V^{\prime}\right|=q^{5}=\left|V^{\prime \prime}\right|$. Then from the representation of $Y$ on $V^{\prime}$, the involutions in $V^{\prime}$ of type $c_{2}$ in $X_{1}$ are in the third class of 2-central involutions in $P_{1}$, i.e. those which are not root involutions. As $u_{c}$ belongs to this class, it follows that $c_{2} \subseteq u_{c}^{L}$.

From 12.1, $X=\hat{X}_{\tau}$ with $b_{1} \subseteq j_{1}, a_{2}, c_{2} \subseteq j_{2}$, and $b_{3} \subseteq j_{3}$. Define $m_{i}$ as in 11.3 (2). Then as $m_{3} \neq m_{1}$ or $m_{2}, b_{3}$ is in the fourth $L$-class $v^{L}$ of involutions of $L$, completing the proof of (3).

Finally (5) follows from (3) and (4).

Lemma 12.4. For $x \in\left\{u_{c}, v\right\}$ let $U_{x}=R t_{L}(x)$. Then for $x \in\left\{u_{c}, v\right\}$ :

(1) There exist $K_{x} \leq X$ with $\left(K_{x}, U_{x}\right) \in \mathfrak{S}(X, x)$.

(2) $\left(K_{x}, U_{x}\right) \in \mathfrak{S}(L, x)$.

(3) $O_{2}\left(C_{G}\left(K_{x}\right)\right)=1$.

Proof. Part (1) was established in 9.16. In particular $K_{v} \cong L_{2}(q), K_{u_{c}} \cong L_{2}\left(q^{2}\right)$, and $x \in U_{x} \cong E_{q}$. From the construction of $K_{x}$ in Section 9, $U_{x}=R t_{\operatorname{Sp}\left(N_{6}\right)}(x)$, and from 12.1, $R t_{\mathrm{Sp}\left(N_{6}\right)}(x)=R t_{\mathrm{SL}\left(N_{6}\right)}(x)$. Thus from 11.3(6), $U_{x}=R t_{\hat{L}}(x) \leq$ $Z\left(C_{L}(x)\right)$. Thus to establish (2), it remains to show that $C_{L}\left(K_{m}\right)$ is a complement to $\mathrm{O}_{2}\left(C_{L}(x)\right)$ in $C_{L}(x)$. Set $R:=O_{2}\left(C_{L}\left(K_{x}\right)\right)$.

First take $x=v$. Then the discussion in Section 9 (or 7.10 in [ASe]) says that $C_{X}\left(K_{v}\right) \cong L_{2}(q)$. Set $B_{v}:=K C_{X}\left(K_{v}\right)$. Then $B_{v} \cong L_{2}(q) \times L_{2}(q)$, and by 13.3 in [ASe], $B_{v}$ is a complement to $O_{2}\left(C_{L}(v)\right)$ in $C_{L}(v)$. Thus to complete the proof of (2) in this case, we may assume $R \neq 1$ and it remains to derive a contradiction. By Borel-Tits, $N_{L}(R) \leq P$ a parabolic of $L$. As $L_{2}(q)^{3} \cong K_{v} B_{v} \leq N_{L}(R)$, we conclude $P$ is a conjugate of $P_{1}$ or $P_{4}$. Thus $K_{v} B_{3}$ centralizes some root group $U_{0}$, so

$$
U_{0} \leq C_{L}\left(K_{v} B_{3}\right) \leq C_{L}(K) \cap C_{L}\left(K_{v} C_{X}\left(K_{v}\right)\right)=C_{X}\left(K_{v} C_{X}\left(K_{v}\right)\right)=1
$$


as $K_{v} \in \subseteq(X, v)$ and $C_{X}\left(K_{v}\right) \cong L_{2}(q)$. This completes the proof of (2) when $x=v$.

Next take $x=u_{c}$. Now (cf. Table 4.1 in [GL]) there exists a subgroup $Y$ of $L$ isomorphic to $\operatorname{Sp}_{8}(q)$ and generated by root groups. Conjugating in $\operatorname{Aut}(L)$ we may assume long root groups of $Y$ are long root groups of $L$, and then that $K$ is such a root group. Then $C_{Y}(K) \cong \operatorname{Sp}_{6}(q) \cong X$, so $C_{Y}(K)=X$. Hence $K_{x} \leq X \leq Y$ and $x$ is of type $c_{2}$ in $Y$ by 12.3. Hence (cf. 9.13), $B_{x}:=C_{Y}\left(K_{x}\right) \cong \operatorname{Sp}_{4}(q)$. Then by 13.3 in [ASe] $B_{x}$ is a complement to $O_{2}\left(C_{L}(x)\right)$ in $C_{L}(x)$. If $R \neq 1$, then by Borel-Tits, $K_{x} B_{x} \leq N_{L}(R) \leq P$ for some proper parabolic $P$ of $L$. This is impossible as no proper parabolic has an $\left(L_{2}\left(q^{2}\right) \times \mathrm{Sp}_{4}(q)\right)$-section. Hence (2) is established.

Finally we prove (3). By (2) we may assume $G \neq L$. From 19.3 in [ASe], $\operatorname{Out}(L)$ is cyclic, and then as $G=L V, 19.5$ in [ASe] says that $G=L\langle t\rangle$ where $t$ is an involution inducing a field or graph-field automorphism on $L$, with $q$ a square in the first case. If $t$ is a field automorphism, then $\operatorname{Aut}_{G}\left(K_{x}\right)$ contains a field automorphism not in $\operatorname{Aut}_{L}\left(K_{x}\right)$, so $C_{G}\left(K_{x}\right) \leq L$. If $t$ is a graph-field automorphism, then from 19.5 in [ASe], $C_{L}(t) \cong{ }^{2} F_{4}(2)$ is of Lie rank 2 , so it does not contain $K_{x} C_{L}\left(K_{x}\right)$ of Lie rank 3 by 7.1, so again $C_{L}\left(K_{x} C_{L}\left(K_{x}\right)\right)=1$.

Lemma 12.5. For each involution $x \in L$, there exist $\left(K_{x}, U_{x}\right) \in \mathfrak{S}(L, x)$, and $\mathrm{O}_{2}\left(C_{G}\left(K_{x}\right)\right)=1$.

Proof. The lemma follows from 10.2 if $x$ is a long root involution, and hence also when $x$ is a short root involution, as long and short root involutions are fused in $\operatorname{Aut}(L)$. Thus by 12.3, we may assume $x \in\left\{u_{c}, v\right\}$, where the lemma follows from 12.4.

\section{3. $E_{7}(q)$}

In this section we assume Hypothesis 10.5 with $L=E_{7}(q)$.

Lemma 13.1. (1) $P(\xi)=P_{1}$. Thus $L_{1}=X \cong \Omega_{12}^{+}(q)$.

(2) $Q_{1} \cong q^{1+32}$ is special with center $U$.

(3) $L_{7} \cong E_{6}(q)$ and $Q_{7} \cong E_{q^{27}}$ is the 27-dimensional $\mathbb{F}_{q}$-module for $L_{7}$, discussed in 11.1. Further $Q_{1} \cap Q_{7} \cong E_{q^{17}}$, and $L_{1,7} \cong \Omega_{10}^{+}(q)$ acts naturally on the complement $W=Q_{7} \cap X=R\left(P_{1,7} \cap X\right)$ to $Q_{1} \cap Q_{7}$ in $Q_{7}$.

Proof. From the discussion in Section 16 in [ASe], $P_{1}=P(\xi), Q_{1} \cong q^{1+32}$, and $L_{1} \cong \Omega_{12}^{+}(q)$. Then $L_{1}=X$ by 10.2 (3), establishing (1) and (2). From the Dynkin diagram for $L, L_{7} \cong E_{6}(q)$, and then as $\left|E_{6}(q)\right|_{2}=q^{36}$ and $\left|E_{7}(q)\right|=q^{63}$, $\left|Q_{7}\right|=q^{27}$. Then as 27 is the minimal dimension of a faithful $L_{7} \mathbb{F}_{q}$-module, and all such modules are quasiequivalent, the first statement in (3) holds. The second follows as the radical of $P_{1,7} \cap X$ is the natural module for $L_{1,7} \cong \Omega_{10}^{+}(q)$. 
Lemma 13.2. (1) L has five classes of involutions.

(2) $P_{7}$ controls fusion in $Q_{7}$.

(3) $P_{7}$ has three orbits on involutions in $Q_{7}$ with representatives $u_{i}, 1 \leq i \leq 3$, denoted by $x, y, u$ in 16.1 of [ASe].

(4) $u_{1} \in U$ and $U$ is a singular point in $Q_{7}$.

(5) $u_{2}$ and $u_{3}$ are in brilliant and dark points of $Q_{7}$, respectively.

(6) Let $W_{1}=U$ and $W_{i}, i=2,3$ be root groups in the orthogonal space $W$ for $L_{1,7}$ in 13.1 (3), which are not orthogonal in that space. Let $w_{i}$ be an involution in $W_{i}$. Then we may pick $u_{1}=w_{1}, u_{2}=w_{1} w_{2}$, and $u_{3}=w_{1} w_{2} w_{3}$.

(7) $C_{L}\left(u_{3}\right)=C_{P_{7}}\left(u_{3}\right)$ contains $Q_{7}$ and $C_{L}\left(u_{3}\right) / Q_{7} \cong F_{4}(q)$.

Proof. Part (1) is 16.1 in [ASe]. As the radical $Q_{7}$ is weakly closed and abelian, (2) follows from 7.7 in [A8].

By 13.1 (3), $Q_{7}$ is the 27-dimensional module for $L_{7} \cong E_{6}(q)$. That module is described in detail in [A5]; we adopt the terminology from [A5] in discussing the module. In particular the first statement in (3) follows from 3.16.1 in [A5], which says $P_{7}$ has three orbits on the $\mathbb{F}_{q}$-points of the $\mathbb{F}_{q}$-module $Q_{7}$, namely the singular, brilliant, and dark points. The torus $H_{K}$ is transitive on vectors in each point. The $\Omega_{10}^{+}(q)$-parabolic $P_{1,7}$ stabilizes a singular point, which is therefore $U$. Thus from 16.20 in [ASe], $u_{1} \in U$ is the involution denoted by $x$ in that lemma. From 13.1 (3), $Q_{1} \cap Q_{7}$ is of dimension 17; this is the subspace $U \Delta$ of [A5]. Let $U=W_{1}$ and pick root groups $W_{i}, i=2,3$ in $Q_{7}$ such that $\left\{W_{1}, W_{2}, W_{3}\right\}$ is special as defined on page 164 of [A5]. In particular $W_{i} \not W_{j} \Delta$ for $i \neq j$. We may choose $W_{i}, i=1,2$, to be root groups in the complement $W$ to $U \Delta$ defined in 13.1 (3). The condition $W_{3} \not W_{2} \Delta$ is equivalent to $W_{2}$ and $W_{3}$ not orthogonal in the orthogonal space $W$ for $L_{1,7}$. Set $u_{2}=u_{1} w_{2}$ for some involution $w_{2} \in W_{2}$; this is the involution denoted by $y$ in 16.20 of [ASe], since $u_{2}$ is diagonal in the product of two commuting root groups $U$ and $W_{2}$ such that $W_{2} \not \leq O_{2}\left(N_{L}(U)\right)$, and (cf. the proof of 12.1 in [ASe]) $L$ is transitive on such involutions. From [A5], $u_{2}$ is contained in a brilliant point of $Q_{7}$.

Finally let $u_{3}=u_{1} w_{2} w_{3}$ for some involution $w_{3} \in W_{3}$. From [A5], $u_{3}$ is contained in a dark point of $Q_{7}$, and hence (cf. 8.14 in [A5]) $C_{P_{7}}\left(u_{3}\right) / Q_{7} \cong F_{4}(q)$. From 16.20 in [ASe], only the centralizer of the involution denoted by $u$ in that lemma contains an $F_{4}(q)$-section, so $u_{3}$ is in that class. Then by 16.19 in [ASe], $C_{L}\left(u_{3}\right)=C_{P_{7}}\left(u_{3}\right)$.

We have verified (1)-(7), so the proof of the lemma is complete.

Lemma 13.3. Let $M$ be the 12-dimensional orthogonal space over $\mathbb{F}_{q}$ for $X$, and $M=M_{1} \perp M_{2} \perp M_{3}$ an orthogonal decomposition with each $M_{i}$ a 4-dimensional nondegenerate subspace of sign -1 . For $1 \leq i \leq 3$, let $J_{i}, J_{i}^{\prime}$ be the fundamental 
subgroups of $X$ with $M_{i}=\left[M, J_{i}\right]=\left[M, J_{i}^{\prime}\right]$, and let $\Delta=\left\{J_{i}, J_{i}^{\prime}, K: 1 \leq i \leq 3\right\}$, and $D=\langle\Delta\rangle$. Then:

(1) $D=\prod_{J \in \Delta} J$ is the direct product of members of $\Delta$ and $N_{L}(D) / D$ acts faithfully as $L_{3}(2)$ on $\Delta$ preserving a projective plane $\Gamma$ on $\Delta$ in which $\left\{K, J_{i}, J_{i}^{\prime}\right\}$, $1 \leq i \leq 3$ are the lines through $K$.

(2) Let $r=r_{1} \ldots r_{7}$ with $r_{i} \in D_{i}, \Delta=\left\{D_{1}, \ldots, D_{7}\right\}$, and $r_{i}^{2}=1$. Set $\delta(r)=$ $\left\{D_{i}: r_{i} \neq 1\right\}$. Then $r$ is L-conjugate to $u_{1}, u_{2}$ if $|\delta(r)|=1,2$ respectively, and is conjugate to $u_{3}$ if $\delta(r)$ is a line in $\Gamma$.

Proof. Working in $K \times X, D$ is the direct product of the seven copies of $L_{2}(q)$ in $\Delta$. Further $X=C_{L}(K)$ and from the structure of $X=\Omega_{12}^{+}(q), C_{X}(D \cap X)=1$, so $C_{L}(D)=1$. Next $N_{X}(D) /(D \cap X)$ acts faithfully as $S_{4}$ on $\Delta-\{K\}$, and preserves the partition $\left\{\left\{J_{i}, J_{i}^{\prime}\right\}: 1 \leq i \leq 3\right\}$, and similarly $N_{L}\left(J_{1}\right) \cap N_{L}(D)$ is transitive on $\Delta-\left\{J_{1}\right\}$, so (1) follows. Then (2) follows from (1) and the description of $u_{i}$ in $13.2(6)$.

Lemma 13.4. Pick $u_{3}$ as in 13.2(6) and notation as in 13.3. Pick $W_{2} \leq J_{1}$ and $W_{3} \leq J_{1}^{\prime}$. Then:

(1) There exists $S_{3} \cong S \leq N_{L}(D) \cap C_{L}\left(u_{3}\right)$ faithful on $\delta:=\left\{K, J_{1}, J_{1}^{\prime}\right\}$ such that the involution $s \in S$ fixing $K$ is in $X$.

(2) Set $K_{3}:=C_{K J_{1} J_{1}^{\prime}}(S)$ and let $u_{3} \in U_{3} \in \operatorname{Syl}_{2}\left(K_{3}\right)$. Then $\left(K_{3}, U_{3}\right) \in$ $\mathfrak{S}\left(L, u_{3}\right)$.

(3) $O_{2}\left(C_{L}\left(K C_{L}(K)\right)\right)=1$.

Proof. Observe that $N_{X}(D)$ is the wreath product of $J_{1}$ with $S_{4}$. Then pick $S$ to be a conjugate of an $S_{3}$-subgroup of a wreath complement to $D \cap X$ in $N_{X}(D)$ such that $S$ is transitive on $\delta$; this is possible by 13.3. This establishes (1).

Let $Y:=C_{L}\left(u_{3}\right)$. By 13.2(7), $Q_{7}=O_{2}(Y)$ and $Y^{*}:=Y / Q_{7} \cong F_{4}(q)$. Next $C_{X}\left(K_{3}\right)=C_{X}\left(K_{s}\right)$, where $K_{s}$ is the projection of $K_{3}$ on $J_{1} J_{1}^{\prime}$. Thus $K_{s}=C_{J_{1} J_{1}^{\prime}}(s)$. From 9.17, $B_{K}:=C_{X}\left(K_{S}\right) \cong \operatorname{Sp}_{8}(q)$. As $S$ is transitive on $\delta$ there is also an $S$ conjugate $B_{1}$ of $B_{X}$ in $C_{L}\left(J_{1}\right)$ centralizing $K_{3}$, and as $B_{K}$ centralizes $W_{1}$ but not $W_{1}$, $B_{X}^{*} \neq B_{1}^{*}$. Then as $B_{X}^{*} \cong \operatorname{Sp}_{8}(q)$ is maximal in $Y^{*} \cong F_{4}(q), Y^{*}=\left\langle B_{X}, B_{1}\right\rangle^{*} \leq$ $Y_{3}^{*}$, where $Y_{3}:=C_{L}\left(K_{3}\right)$. Set $R:=O_{2}\left(Y_{3}\right)$. Then $R \leq Q_{7}$ and $U_{3} \leq C_{Q_{7}}\left(Y_{3}\right)$, so as $C_{Q_{7}}\left(Y_{3}\right)$ is of order $q$ and $Y_{3}$ is irreducible on $Q_{7} / C_{Q_{7}}\left(Y_{3}\right)$, it follows that $U_{3}=Z(Y)$ and either $R=1$ or $R$ is a complement to $U_{3}$ in $Q_{7}$. But in the latter case $K_{3}$ acts on $Q_{7}=C_{L}(R)$, contradicting $U_{3} \leq Q_{7}$. This completes the proof of (2).

Finally from 19.2 in [ASe], all involutions in $\operatorname{Aut}(L)-L$ are field automorphisms, and such an involution induces a field automorphism on $Y^{*}$, so (3) holds. 
Lemma 13.5. (1) $L_{6} \cong L_{2}(q) \times \Omega_{10}^{+}(q)$ and $Q_{6} \cong q^{10+32}$ is special.

(2) $Z_{6}=Z\left(Q_{6}\right) \cong E_{q_{10}}$ admits the structure of a 10-dimensional orthogonal space over $\mathbb{F}_{q}$, preserved by $L_{6}$, and $L_{6}$ induces $\Omega_{10}^{+}(q)$ on this space.

(3) We may take $u_{2}=u_{1} w_{2}$ where $W_{2}$ is the root group of $X$ such that $X_{6}=$ $X \cap P_{6}=N_{X}\left(W_{2}\right)$, and $w_{2} \in W_{2}$.

(4) $u_{2}$ is a nonsingular point in the orthogonal space $Z_{6}$ and $C_{L}\left(u_{2}\right)=Q_{6} C_{L_{6}}\left(u_{2}\right)$ with $C_{L_{6}}\left(u_{2}\right) \cong L_{2}(q) \times \operatorname{Sp}_{8}(q)$.

(5) Let $W_{2} \leq J_{1}$, s an involution in $N_{L}(\Delta)$ with cycle $\left(K, J_{1}\right), K_{2}=C_{K J_{1}}(s)$, and $u \in U_{2} \in \operatorname{Syl}_{2}\left(K_{2}\right)$.Then $\left(K_{2}, U_{2}\right) \in \mathfrak{S}\left(L, u_{2}\right)$.

(6) $\mathrm{O}_{2}\left(C_{G}\left(K_{2}\right)\right)=1$.

Proof. Parts (1) and (2) are established during the discussion on page 60 of [ASe]. Part (3) follows from 13.2(6). Arguing as usual, $R\left(X_{6}\right) \cong q^{1+12}$ is a complement to $Q_{1} \cap Q_{6}$ in $Q_{6}$, so $W_{2}=\Phi\left(R\left(X_{6}\right)\right) \leq \Phi\left(Q_{6}\right)=Z_{6}$. Indeed $W_{2}$ is a complement to $Q_{1} \cap Z_{6}$ in $Z_{6}$, and $Q_{1} \cap Z_{6}$ is the subspace of the orthogonal space $Z_{6}$ orthogonal to $U$. Thus $u_{2}=u_{1} w_{2}$ is a nonsingular point in $Z_{6}$, so $C_{L_{6}}\left(u_{2}\right) \cong L_{2}(q) \times \operatorname{Sp}_{8}(q)$. From $13.2(3), u_{2}$ is the involution $y$ of 16.1 in [ASe], so from the description of $C_{L}(y)$ in 16.20 of [ASe], $Y:=C_{L}\left(u_{2}\right)=C_{P_{6}}\left(u_{2}\right)$. Thus (4) holds.

Adopt the notation of (5). Then $C_{X}\left(K_{2}\right)=C_{X}\left(J_{1}\right) \cong L_{2}(q) \times \Omega_{8}^{+}(q)$ by 10.2 (3). Then $C_{X}\left(K_{2}\right)$ and $Y^{*}:=Y / Q_{6} \cong L_{2}(q) \times \operatorname{Sp}_{8}(q)$ have the same Lie rank, so $O_{2}\left(C_{L}\left(K_{2}\right)\right)=1$ by 10.6 .

Next by 13.3, there is $g \in N_{L}(\Delta)$ with $K_{2}^{g} \leq J_{1} J_{1}^{\prime}$. Then $u_{2}^{g}$ is of type $c_{2}$ in $X$ and $s^{g} \in X$ centralizes $K_{2}^{g}$, so by $9.14, C_{X}\left(K_{2}^{g}\right) \cong \operatorname{Sp}_{8}(q)$. It follows from (4) that $K C_{X}\left(K_{2}\right)$ is a complement to $Q_{6}$ in $C_{L}\left(u_{2}\right)$. Further from the action of $B:=C_{L_{6}}\left(u_{2}\right)$ on $Q_{6}, C_{Q_{6}}(B) \leq Z_{6}$, so $U_{3} \leq Z\left(C_{L}\left(u_{2}\right)\right)$, completing the proof of (5).

Recall from 19.3 in [ASe] that each involution in $\operatorname{Aut}(L)-L$ induces a field automorphism on $L$, and hence also on $Y^{*}$, so (6) follows.

\section{4. $E_{8}(q)$}

In this section we assume Hypothesis 10.5 with $L=E_{8}(q)$.

Lemma 14.1. (1) $P(\xi)=P_{8}$. Thus $L_{8}=X \cong E_{7}(q)$.

(2) $Q_{8} \cong q^{1+56}$ is special with center $U$ and $Q_{8} / U$ is the faithful 56-dimensional $\mathbb{F}_{q} X$-module.

Proof. From the discussion in Section 17 in [ASe], $P_{8}=P(\xi), Q_{8} \cong q^{1+56}$, and $L_{8} \cong E_{7}(q)$. Then $L_{8}=X$ by 10.2 (3), establishing (1). Up to quasiequivalence, $E_{7}(q)$ has a unique faithful 56-dimensional $\mathbb{F}_{q}$-module, so (2) follows. 
Lemma 14.2. (1) L has four classes of involutions.

(2) Each involution in $Q_{8}$ is conjugate in $L$ to $u_{i}$ for some $1 \leq i \leq 3$, denoted by $x, y, z$ in 17.1 of [ASe], respectively.

(3) $u_{1} \in U$.

Proof. Part (1) is 17.1 in [ASe]. Let $\widetilde{Q}_{8}=Q_{8} / U$. By 14.1(2), $\widetilde{Q}_{8}$ is the 56dimensional module for $X \cong E_{7}(q)$. The orbits of $X$ on $\widetilde{Q}_{8}$, and centralizers in $X$ of representatives, are listed in 4.3 of [LS]. In particular if $i \in Q_{7}-U$ is an involution, then each nonabelian composition factor of $C_{X}(\tilde{i})$ is a section of $C_{L}(i)$. We compare the factors from [LS] of $C_{X}(\tilde{i})$ to the factors of $C_{L}(i)$ in 17.15 of [ASe], and conclude only conjugates of $x, y$, and $z$ can be contained in $Q_{8}$, since the only nonabelian composition factor of $C_{L}(u)$ is $\operatorname{Sp}_{8}(q)$, which does not contain the nonabelian composition factor of $C_{X}(j)$ for any $j \in \widetilde{Q}_{8}$. Thus (2) holds.

From the definition of $x$ in Section 17 of [ASe], $x=u_{1}$ is a root element, so (3) holds.

Lemma 14.3. Let $\Delta^{\prime}$ be the set of seven fundamental subgroups of $X$ defined in 13.3 and set $\Delta:=\Delta^{\prime} \cup\{K\}$. For $1 \leq i \leq 3$ let $I_{i}$ be the set of involutions in $\langle\Delta\rangle$ projecting nontrivially on exactly $i$ members of $\Delta$. Then $N_{L}(\Delta)$ is 3-transitive on $\Delta$ and transitive on $I_{i}$ for each $1 \leq i \leq 3$.

Proof. From 13.3, $N_{X}\left(\Delta^{\prime}\right)$ is 2-transitive on $\Delta^{\prime}$, so $N_{L}(\Delta)$ is 3-transitive on $\Delta$ by our usual argument. Hence the lemma follows.

Lemma 14.4. (1) $L_{1} \cong \Omega_{14}^{+}(q)$ and $Q_{1} \cong q^{14+64}$ is special.

(2) $Z_{1}=Z\left(Q_{1}\right) \cong E_{q^{14}}$ admits the structure of a 14-dimensional orthogonal space over $\mathbb{F}_{q}$, preserved by $L_{1}$, and $L_{1}$ induces $\Omega_{14}^{+}(q)$ on this space.

(3) We may take $u_{2}=u_{1} w_{2}$ where $W_{2}$ is the root group of $X$ such that $X_{1}=$ $X \cap P_{1}=N_{X}\left(W_{2}\right)$, and $w_{2} \in W_{2}$. In particular, in the language of 14.3 , we may choose $W_{2} \leq J \in \Delta^{\prime}$, so $u_{2} \in I_{2}$.

(4) $u_{2}$ is a nonsingular point in the orthogonal space $Z_{1}$ and $C_{L}\left(u_{2}\right)=Q_{1} C_{L_{1}}\left(u_{2}\right)$ with $C_{L_{1}}\left(u_{2}\right) \cong \operatorname{Sp}_{12}(q)$.

(5) Let $K_{2}=C_{K J}(s)$ for $s$ an involution in $N_{L}(\Delta)$ with cycle $(K, J)$, and $u_{2} \in$ $U_{2} \in \operatorname{Syl}_{2}\left(K_{2}\right)$. Then $\left(K_{2}, U_{2}\right) \in \mathfrak{S}\left(L, u_{2}\right)$.

(6) $\mathrm{O}_{2}\left(C_{G}\left(K_{2}\right)\right)=1$.

Proof. Parts (1) and (2) are established during the discussion on page 69 of [ASe].

From 14.2(2), $u_{2}$ is conjugate to the involution $y$ of 17.1 in [ASe], and from the definition of $y$ in Section 17 of [ASe], $u_{2}$ is diagonal in the product of two commuting root groups $U$ and $W_{2}$ such that $W_{2} \not \leq O_{2}\left(N_{L}(U)\right)$. Thus as $L$ is 
transitive on such involutions (cf. the proof of 12.1 in [ASe]), we may choose $u_{2}$ as in (3). Arguing as usual, $R\left(X_{1}\right) \cong q^{1+32}$ is a complement to $Q_{1} \cap Q_{8}$ in $Q_{1}$, so $W_{2}=\Phi\left(R\left(X_{1}\right)\right) \leq \Phi\left(Q_{1}\right)=Z_{1}$. Indeed $W_{1}$ is a complement to $Q_{8} \cap Z_{6}$ in $Z_{1}$, and $Q_{8} \cap Z_{6}$ is the subspace of the orthogonal space $Z_{1}$ orthogonal to $U$. Thus $u_{2}=u_{1} w_{2}$ is a nonsingular vector in $Z_{1}$, so $C_{L_{1}}\left(u_{2}\right) \cong \operatorname{Sp}_{12}(q)$. From the description of $C_{L}(y)$ in 17.15 of [ASe], $Y:=C_{L}\left(u_{2}\right)=C_{P_{1}}\left(u_{2}\right)$. Thus (4) holds.

Define $\left(K_{2}, U_{2}\right)$ as in (5) and let $B_{2}:=C_{L}\left(K_{2}\right)$. Then $C_{X}\left(K_{2}\right)=C_{X}(J) \cong$ $\Omega_{12}^{+}(q)$ is of Lie rank 6 , as is $Y^{*}:=Y / O_{2}(Y) \cong \operatorname{Sp}_{12}(q)$, so $O_{2}\left(B_{2}\right)=1$ by 10.6. By 14.3, there is $g \in N_{L}(\Delta)$ with $K_{2}^{g} \leq J J_{2}$ for some $J_{2} \in \Delta^{\prime}-\{J\}$. As $K^{g}=$ $C_{J J^{g}}\left(s^{g}\right), K^{g} \in \mathfrak{S}\left(X, u_{2}^{g}\right)$ so $X_{2}:=C_{X}\left(K_{2}^{g}\right) \cong L_{2}(q) \times \operatorname{Sp}_{8}(q)$ by 13.5. Then $B:=\left(K X_{2}\right)^{g^{-1}} \leq B_{2}$ and $N_{Y^{*}}\left(C_{X}\left(K_{2}\right)^{*}\right) \cong O_{12}^{+}(q)$ is the unique maximal overgroup in $Y^{*}$ of $C_{X}\left(K_{2}\right)^{*} \cong \Omega_{12}^{+}(q)$. Hence as $O_{12}^{+}(q)$ contains no copy of $B \cong L_{2}(q)^{2} \times \operatorname{Sp}_{8}(q)$, it follows that $B_{2}=C_{L}\left(K_{2}\right)$. As $C_{Q_{1}}\left(C_{L_{1}}\left(u_{2}\right)\right)$ is of order $q$ it follows that $U_{2}=C_{Q_{1}}\left(B_{2}\right)=Z\left(C_{L}\left(u_{2}\right)\right)$, so (5) holds. Finally by 19.2 in [ASe], each involution in $\operatorname{Aut}(L)-L$ induces a field automorphism on $L$, so (6) follows as usual.

Lemma 14.5. Let $g \in P_{7}-P_{8}, I=U U^{g}, E=Q_{8} \cap Q_{8}^{g}$, and $J=\left\langle Q_{8}, Q_{8}^{g}\right\rangle$. Then:

(1) $Q_{7}=\left(Q_{8} \cap Q_{7}\right)\left(Q_{8}^{g} \cap Q_{7}\right)$ and $J / Q_{7} \cong L_{2}(q)$.

(2) $L_{7}=\left(J \cap L_{7}\right) \times L_{7,8}$ with $J_{7}=J \cap L_{7} \cong L_{2}(q)$ and $L_{7,8} \cong E_{6}(q)$.

(3) $I=Z\left(Q_{7}\right)$ is the natural module for $J_{7}$.

(4) $[J, E]=I$ and $E / I$ is the 27-dimensional module for $L_{7,8}$.

(5) $Q_{7} / E$ is the tensor product of the natural module for $J_{7}$ and the dual of $E / I$ for $L_{7,8}$.

(6) We may choose $u_{3} \in E-I$, such that $C_{P_{7}}\left(u_{3}\right)=C_{L}\left(u_{3}\right), C_{L_{7}}\left(u_{3}\right)=J_{7} \times$ $C_{L_{7,8}}\left(u_{3}\right)$, and $C_{L_{7,8}}\left(u_{3}\right) \cong F_{4}(q)$.

(7) There exists $K_{3} \in \mathfrak{S}\left(L, u_{3}\right)$.

(8) $\mathrm{O}_{2}\left(C_{G}\left(K_{3}\right)\right)=1$.

Proof. The proof is similar to that of 11.6. Parts (1)-(5) follow from the standard theory of large special groups; see for example 8.15 in [A8]. By 14.2 (2), $u_{3}$ is fused to the involution $z$ of 17.1 in [ASe]. Thus from 17.14 in [ASe], we may take $C_{L}\left(u_{3}\right) \leq P_{7}$ with $O_{2}\left(C_{L}\left(u_{3}\right)\right)=C_{Q_{7}}\left(u_{3}\right)$, and $O^{2^{\prime}}\left(C_{L}\left(u_{3}\right)\right) / O_{2}\left(C_{L}\left(u_{3}\right)\right)$ as described in (6). From the action of $L_{s}$ on $Q_{s}$ described in (1)-(5), it follows that $u_{3} \in E$, completing the proof of (6).

Let $X_{7}=X \cap P_{7}$ and $W=R\left(X_{7}\right)$. As $L_{7,8} \cong E_{6}(q)$, from 13.2 (7) there is an involution $v_{3} \in W$ such that $C_{X}\left(v_{3}\right)=C_{X_{7}}\left(v_{3}\right)$ with $C_{X}\left(v_{3}\right) / W \cong F_{4}(q)$. Further by 13.4 , there is $K_{v} \in \mathfrak{S}\left(X, v_{3}\right)$. Thus $C_{L}\left(K_{v}\right)$ contains $K C_{X}\left(K_{v}\right) \cong L_{2}(q) \times F_{4}(q)$. 
Now from the structure of $C_{X}\left(v_{3}\right)$ and the list of centralizers in 17.15 in [ASe], $v_{3} \in u_{3}^{L}$. Thus there exists $g \in L$ with $v_{3}^{g}=u_{3}$. Let $K_{3}:=K_{v}^{g}$. We have shown that $C_{L}\left(K_{3}\right)$ contains a subgroup $Y=K_{4} \times Y_{0}$ where $K_{4}=K^{g}$ is a fundamental subgroup of $L$, and $Y_{0}=C_{X}\left(K_{v}\right)^{g} \cong F_{4}(q)$. Hence $K_{4} Y_{0}$ is a complement to $\mathrm{O}_{2}\left(C_{L}\left(u_{3}\right)\right)$ in $C_{L}\left(u_{3}\right)$ by $(6)$.

Suppose $O_{2}\left(C_{L}\left(K_{3}\right)\right)=R \neq 1$. As the complement $Y$ to $O_{2}\left(C_{L}\left(u_{3}\right)\right)$ acts on $R, R \leq Q_{7}$. From the description of the action of $L_{7}$ on $Q_{7}$ in (1)-(5) and the description of the action of $E_{6}$ on its 27-dimensional module in [A5]:

(i) $K_{4}$ is irreducible on $I$.

(ii) $\widetilde{E}=E / I=\widetilde{W}^{g}$, and $W^{g}=U_{3} \times W_{3}$ and $Y_{0}$ is irreducible on $W_{3}$.

(iii) $Q_{7}^{*}=Q_{7} / E=C_{Q_{7}}\left(u_{3}\right)^{*} \times Q^{*}$ where $\left|Q^{*}\right|=q^{2}$ and $Y$ is irreducible on both factors.

We argue as in the proof of 11.6 to derive a contradiction: First $I \cap R=1$ by (i) and as $K_{3} \not \leq P_{7}=N_{L}(I)$. Second, as all $Y$-submodules of $E$ not containing $I$ either are contained in $U_{3}$ or contain $W_{3}, R \cap E=1$ as $R \cap W^{g}=1$. Therefore third, by (iii), $R^{*}=C_{Q_{7}}\left(u_{3}\right)^{*}$, so as $\Phi\left(C_{Q_{7}}\left(u_{3}\right)\right) \leq E, C_{Q_{7}}\left(u_{3}\right)=R$, a contradiction.

Hence $O_{2}\left(C_{L}\left(K_{3}\right)\right)=1$. Moreover we have also shown that $U_{3}=Z\left(C_{L}\left(u_{3}\right)\right)$, so (7) holds. Finally (8) follows from (7) as usual.

\section{The proof of Theorem 4}

In this section we complete the proof of Theorem 4. Thus we assume the hypothesis of that Theorem: $G=O^{2^{\prime}}(G)$ is an almost simple group of Lie type over $\mathbb{F}_{q}$, or the Tits group. Set $L:=F^{*}(G)$ and let $i$ be an involution in $L$.

If $L$ is a classical group, then Theorem 4 holds by Theorem 9.17. Thus we may assume $L$ is exceptional. In particular the pair $(G, L)$ satisfies Hypothesis 10.1, with $l$ the Lie rank of $L$.

If $l=1$ then $L \cong S z(q)$, a case handled in 10.3. Then 10.4 handles the case $l=2$. Thus we may assume $l>3$, so $L$ satisfies Hypothesis 10.5 .

If $i$ is a long root involution, then the pair $(L, i)$ satisfies Theorem 4 by 10.2 (4). Suppose $L$ is $E_{6}^{\varepsilon}(q)$. Then by $11.2, L$ has three classes of involutions with representatives $u_{i}, 1 \leq i \leq 3$. The involution $u_{1}$ is a root involution, a case already treated, while Theorem 4 holds for the involutions $u_{2}$ and $u_{3}$ by 11.5 and 11.6.

Theorem 4 holds when $L$ is $F_{4}(q)$ by 12.5 . Suppose $L$ is $E_{7}(q)$. By $13.2(1), L$ has five classes of involutions, described in 16.1 of [ASe]. The classes with representatives $z$ and $v$ appear in case (4) of Theorem 4. By 13.2 (3), the remaining classes have representatives $u_{i}, 1 \leq i \leq 3$, corresponding to the classes $x, y, u$ of 16.1 in [ASe], respectively. By $13.2(4), u_{1}$ is a root involution, while Theorem 4 holds for $u_{2}$ and $u_{3}$ by 13.5 and 13.4 .

This leaves the case $L \cong E_{8}(q)$. By 14.2(1), $L$ has four classes of involutions, 
described in 17.1 of [ASe]. The class with representative $v$ appears in case (5) of Theorem 4. By 14.2 (2), the remaining classes have representatives $u_{i}, 1 \leq i \leq 3$, corresponding to the classes $x, y, z$ of [ASe]. By 14.2(3), $u_{1}$ is a root involution, while Theorem 4 holds for $u_{2}$ and $u_{3}$ by 14.4 and 14.5.

This completes the proof of Theorem 4 .

\section{The proof of Theorem 2}

In this section we complete the proof of Theorem 2. Thus we assume the pair $(G, V)$ is a minimal counter example to Theorem 2, as defined in Section 1. Then by Proposition 1.14:

Lemma 16.1. $G$ is almost simple and $G=L V$, where $L=F^{*}(G)$.

Lemma 16.2. L is group of Lie type over $\mathbb{F}_{q}$, for some power $q$ of 2 . Hence the pair $(G, L)$ satisfies Hypothesis 10.1.

Proof. By Theorem 2.1, $G$ is not an alternating or symmetric group. By Theorem 4.1, $L$ is not sporadic. Finally by Theorem 8.3(3), $L$ is not a group of Lie type and odd characteristic. Thus the lemma follows from the classification of the finite simple groups.

Lemma 16.3. $L$ is not $L_{3}(2), L_{4}^{\varepsilon}(2), \mathrm{Sp}_{4}(2)^{\prime}$, or $G_{2}(2)^{\prime}$.

Proof. As $A_{8} \cong L_{4}(2), L$ is not $L_{4}(2)$ by 2.1 . Similarly as $L_{3}(2) \cong L_{2}(7)$, $\mathrm{Sp}_{4}(2)^{\prime} \cong L_{2}(9), G_{2}(2)^{\prime} \cong U_{3}(3)$, and $U_{4}(2) \cong \mathrm{PSp}_{4}(3), L$ is none of these groups by 8.3 .

Lemma 16.4. (1) $V \cap L \neq 1$.

(2) Suppose $i$ is an involution in $L,(K, U) \in \subseteq(L, i)$, and $O_{2}\left(C_{G}(K)\right) \leq L$. Then $i \notin V$.

(3) There exists an involution $u \in V \cap L$ such that for each $K \in \mathbb{S}(L, u)$, $\mathrm{O}_{2}\left(C_{G}(K)\right) \not \leq L$.

Proof. From 2.5.12 in [GLS3], $m_{2}(\operatorname{Out}(L)) \leq 2$. On the other hand, by 1.4 we have $m_{2}(V)>2$, so (1) holds.

Assume the hypothesis of (2). Then from the definition of $\mathbb{S}(L, i)$ in the Introduction, one of the following holds:

(a) $K \cong L_{2}(q)$ or $S z(q)$, or

(b) $K \cong L_{2}\left(q^{2}\right)$ with $q>2$, or

(c) $q=2$ and $K \cong D_{10}$, or 
(d) $i$ is of type $c_{2}$ in $\operatorname{Sp}_{n}(2)$ or $F_{4}(2)$ and $K \cong L_{2}(4)$.

Pick $D$ to be a dihedral subgroup of $K$ of order $2 m$ containing $u$, where $m>1$ is odd in case (a), $m=q^{2}+1$ in case (b), and $m=5$ in case (c) and (d). We first claim that $C_{G}(D)=C_{G}(K)$. In case (d) this holds by case (iii) of the definition of $\subseteq(L, i)$. In case (c) $D=K$. In the remaining cases, $K=\langle U, D\rangle$ and $C_{L}(i)=C_{L}(U)$, so the claim holds in those cases too.

Next, by definition of $\subseteq(L, i), H=C_{L}(K)$ is a complement to $O_{2}\left(C_{L}(i)\right)$ in $C_{L}(i)$, so $O_{2}(H)=1$. Thus as $O_{2}\left(C_{G}(K)\right) \leq L$, also $O_{2}\left(C_{G}(K)\right)=1$. Then $\mathrm{O}_{2}\left(C_{G}(D)\right)=1$ by the claim, so (2) follows from $1.15(1)$.

Finally (1) and (2) imply (3).

Lemma 16.5. (1) $V$ acts on a parabolic $P$ of $L$, where

(i) $P$ is the stabilizer of a singular point in the natural module for $L$ if $L$ is an orthogonal group $\Omega_{n}^{\varepsilon}(q), n \geq 8$

(ii) $P$ is a maximal $V$-invariant parabolic of $L$ if $L$ is $\operatorname{Sp}_{4}(q)$ or $F_{4}(q)$ and $G$ is nontrivial on the Dynkin diagram of $L$;

(iii) $O^{2^{\prime}}(P / R(P)) \cong E_{6}(q)$ if $L$ is $E_{7}(q)$;

(iv) $P=N_{L}\left(U_{\alpha}\right)$ for $\alpha \in \Phi$ a long root in the remaining cases.

(2) $V \cap R(P) \neq 1$.

(3) If $L \cong \operatorname{Sp}_{4}(q)$ and $G$ is nontrivial on the Dynkin diagram of $L$ then all involutions in $V-L$ are graph-field involutions, and all those in $V \cap L$ are of type $c_{2}$.

(4) If $L \cong L_{n}^{\varepsilon}(q)$, then $V$ contains an involution of type $j_{2}$.

(5) If $L$ is symplectic and $G$ is trivial on the Dynkin diagram of $L$, or if $L \cong \Omega_{n}^{\varepsilon}(q)$ with $n \geq 8$, then $V$ contains an involution of type $c_{2}$.

Proof. The normalizer in $G$ of the parabolic listed in (1) contains a Sylow 2-subgroup of $G$, so (1) follows.

Because $l>1$ and the groups in 16.3 are excluded by that lemma, by inspection, either $L \cong L_{3}(4)$ or $O_{2}\left(N_{G}(P)\right)=O_{2}(P)=R(P)$. In the former case, (2) follows from $16.4(1)$, and in the latter case, (2) follows from 1.10 (1).

In the remaining parts, we adopt the notation for involutions in $L$ from earlier sections, and for outer involutions from [GLS3]. Assume the hypothesis of (3). Then since $G=L V, V \not \leq L$. Now (cf. 19.5 in [ASe]) all involutions in $G-L$ are graph-field automorphisms, so $V$ contains a graph-field automorphism $\tau$. Then as $C_{L}(\tau) \cong S z(q)$ has all involutions in $c_{2}$, (3) follows.

We claim that $V$ contains no long root involutions, and if $L$ is symplectic, $V$ contains no short root involutions. For if $i$ is such an involution, then by 10.2 (4) there is $(K, U) \in \subseteq(L, i)$, so $O_{2}\left(C_{G}(K)\right) \not \leq L$ by $16.4(2)$. Then by $10.2(5)$ and 16.3, $L \cong L_{3}(4)$ and $O_{2}\left(C_{G}(K)\right)=\langle\tau\rangle$, where $\tau$ induces a graph automorphism on $L$. But $\left\langle\tau^{C_{L}(i)}\right\rangle$ is not elementary abelian, contrary to 1.15 . Thus the claim is established. 
In cases (4) and (5), we will show that all other involutions in $R=R(P)$ are of type $j_{1}$ and $c_{2}$, in the respective case, to complete the proof of the lemma. Namely let $M$ be the natural module for $\hat{L}$, the covering group of $L$, and identify involutions $v$ in $L$ with those in $\hat{L}$, so that we can consider $\operatorname{dim}([M, v])$. If $L \cong L_{n}(q)$ then $R$ is generated by transvections, so $\operatorname{dim}([M, r]) \leq 2$ for all $r \in R$. Then as $j_{m}$ is the class of involutions $j$ with $\operatorname{dim}([M, j])=m$, and the root involutions are the transvections, the lemma follows in this case. In the remaining cases, $P$ is the parabolic stabilizing a singular point $M_{0}$ of $M$, and $R$ centralizes the chain $0<M_{0}<M_{0}^{\perp}<M$, so $\operatorname{dim}([M, r]) \leq 2$ for $r \in R$. Moreover from Section 9, the involutions $j$ in $L$ with $\operatorname{dim}([M, j]) \leq 2$ are of type $j_{1}$ and $j_{2}$ if $L$ is unitary, and of type $b_{1}, a_{2}$, and $c_{2}$ if $L$ is symplectic or orthogonal, with no involutions in $L$ of type $b_{1}$ when $L$ is orthogonal. Further when $L$ is unitary, the long root involutions are the transvections, when $L$ is symplectic the root involutions are those of type $b_{1}$ and $a_{2}$, while if $L$ is orthogonal the root involutions are of type $a_{2}$. Hence (4) and (5) follow.

Lemma 16.6. L is exceptional.

Proof. Assume otherwise; then $L$ is classical. If $L \cong \operatorname{Sp}_{4}(q)$ and $V$ is nontrivial on the Dynkin diagram, then by $16.5(3), V$ contains an involution $u$ of type $c_{2}$ and each involution in $G-L$ is a graph-field automorphism. Then by Theorem 4, there is $(K, U) \in \mathfrak{S}(L, u)$. From the proof of $16.4(2), C_{G}(K)=C_{G}(D)$ for a suitable dihedral subgroup $D$ of order $2 m$ containing $u$ with $m$ odd, so by 1.15 , there is $t \in V-L$ centralizing $K$. This is impossible as $t$ induces a graph-field automorphism, so $C_{L}(t) \cong S z(q)$, and $S z(q)$ contains no copy of $L_{2}\left(q^{2}\right)$.

Thus the hypothesis of part (4) or (5) of 16.5 is satisfied, so from that lemma, $V$ contains $u$ of type $j_{2}$ or $c_{2}$. By Theorem 4 , there is $(K, U) \in \mathfrak{S}(L, u)$, and from the discussion in Section $9, O_{2}\left(C_{G}(K)\right) \leq L$ unless $L \cong L_{4}^{\varepsilon}(q), L_{n}(2), n \in\{5,6\}$, or $\Omega_{n}^{\varepsilon}(q)$, and $C_{G}(K J)=\langle\tau\rangle$, where $J=C_{L}(K)$ and $\tau$ is a graph automorphism of $L$ in the first two cases, and a transvection in $O_{n}^{\varepsilon}(q)$ in the last case. By $1.15, \tau \in V$.

As $L_{4}^{\varepsilon}(q) \cong \Omega_{6}^{\varepsilon}(q)$, we can subsume this case in the last case. So consider the last case. From 9.18, $\tau \in D \cong D_{2\left(q^{2}+1\right)}$ with $C_{G}(D) \cong O_{n-2}^{-\varepsilon}(q)$, so 1.15 contradicts $\tau \in V$.

Similarly if $L \cong L_{n}(2)$ for $n=5$ or 6 , then by $9.19, \tau \in D \cong D_{2 m}$ with $m=31$ or 7 , and $C_{G}(D) \cong 1$ or $\operatorname{Aut}\left(L_{2}(8)\right)$, for $n=5$, 6, respectively, contrary to 1.15 . This completes the proof.

Lemma 16.7. $V$ does not contain a long root involution.

Proof. Let $u$ be a long root involution. By $10.2(3)$ there is $(K, U) \in \mathfrak{S}(L, u)$, and by 16.5 and $10.2(5), O_{2}\left(C_{G}(K)\right)=1$. Now $16.4(2)$ completes the proof.

Lemma 16.8. L is not exceptional. 
Proof. Assume $L$ is exceptional. Suppose first $L$ is not $E_{7}(q)$ or $E_{8}(q)$. In this case we show:

(a) For each involution $u$ of $L$, which is not a long root involution, there is $K \in$ $\mathfrak{S}(L, u)$ with $\mathrm{O}_{2}\left(C_{G}(K)\right)=1$.

Observe that this suffices by 16.4 (3) and 16.7.

If $l=1$, then $L \cong S z(q)$ and all involutions in $L$ are long root involutions, so (a) holds trivially. If $l=2$, then (a) follows from 10.4. If $L$ is $F_{4}(q)$ then (a) follows from 12.5. Finally if $L$ is $E_{6}^{\varepsilon}(q)$ then by $11.3(1)$ all involutions of $L$ which are not root involutions are conjugate to $u_{2}$ or $u_{3}$, so (a) follows from 11.5 and 11.6(7).

Therefore $L$ is $E_{7}(q)$ or $E_{8}(q)$. Adopt the notation in 10.5. Let $P=P_{l}$ and $Q=Q_{l}$. Then $N_{G}(P)=N_{G}(Q)$ contains a Sylow 2-subgroup of $G$, so we may take $V \leq N_{G}(Q)$, and then as $Q=O_{2}\left(N_{G}(P)\right), V \cap Q \neq 1$ by $1.10(1)$. Then by $16.4(2)$ and 16.7 , it suffices to show:

(b) For each involution $u \in Q$ which is not a root involution, there is $K \in \mathfrak{S}(L, u)$ with $\mathrm{O}_{2}\left(C_{G}(K)\right) \leq L$.

From 13.2(3) and 14.2(2), the involutions in $Q$ are conjugate to $u_{i}$ for some $1 \leq i \leq 3$. Further $u_{1}$ is a root involution. Then 13.4, 13.5, 14.4 and 14.5 show that (b) holds. This completes the proof of the lemma.

Observe that 16.6 and 16.8 supply a contradiction, which completes the proof of Theorem 2.

Acknowledgment. The referee report consisted of six pages of detailed, useful comments. We thank and applaud the referee for this work.

\section{References}

[Al] J. Alperin, On fours groups. Illinois J. Math. 16 (1972), 349-351. Zbl 0253.20060 MR 0291268

[A1] M. Aschbacher, A characterization of Chevalley groups over fields of odd order. Ann. of Math. (2) 106 (1977), 353-398; part II ibid., 399-468; correction ibid. 111 (1980), 411-414. Zbl 0393.20011 Zbl 0429.20020 MR 0498828 MR 0498829 MR 0569077

[A2] M. Aschbacher, On finite groups of Lie type and odd characteristic. J. Algebra 66 (1980), 400-424. Zbl 0445.20008 MR 593602

[A3] M. Aschbacher, On the maximal subgroups of the finite classical groups. Invent. Math. 76 (1984), 469-514. Zbl 0537.20023 MR 746539

[A4] M. Aschbacher, Finite group theory. Cambridge Stud. Adv. Math. 10, Cambridge University Press, Cambridge 1986. Zbl 0583.20001 MR 0895134

[A5] M. Aschbacher, The 27-dimensional module for $E_{6}$. I. Invent. Math. 89 (1987), 159-195. Zbl 0629.20018 MR 892190 
[A6] M. Aschbacher, The 27-dimensional module for $E_{6}$, IV. J. Algebra 131 (1990), 23-39. Zbl 0698.20031 MR 1054997

[A7] M. Aschbacher, Simple connectivity of p-group complexes. Israel J. Math. 82 (1993), 1-43. Zbl 0829.20031 MR 1239044

[A8] M. Aschbacher, Sporadic groups. Cambridge Tracts in Math. 104, Cambridge University Press, Cambridge 1994. Zbl 0804.20011 MR 1269103

[A9] M. Aschbacher, 3-transposition groups. Cambridge Tracts in Math. 124, Cambridge University Press, Cambridge 1997. Zbl 0883.20010 MR 1423599

[ASe] M. Aschbacher and G. M. Seitz, Involutions in Chevalley groups over fields of even order. Nagoya Math. J. 63 (1976), 1-91; correction ibid 72 (1978), 135-136. Zbl 0359.20014 Zbl 0391.20010 MR 0422401 MR 0514895

[CKS] C. W. Curtis, W. M. Kantor, and G. M. Seitz, The 2-transitive permutation representations of the finite Chevalley groups. Trans. Amer. Math. Soc. 218 (1976), 1-59. Zbl 0374.20002 MR 0422440

[GL] D. Gorenstein and R. Lyons, The local structure of finite groups of characteristic 2 type. Mem. Amer. Math. Soc. 42 (1983). Zbl 0519.20014 MR 690900

[GLS3] D. Gorenstein, R. Lyons, and R. Solomon, The classification of the finite simple groups, Number 3. Part I, chapter A: almost simple $K$-groups, Math. Surveys Monogr. 40.3, Amer. Math. Soc., Providence, RI, 1998. Zbl 0890.20012 MR 1490581

[GMS] R. L. Griess, Jr., U. Meierfrankenfeld, and Y. Segev, A uniqueness proof for the Monster. Ann. of Math. (2) 130 (1989), 567-602. Zbl 0691.20014 MR 1025167

[LS] M.W. Liebeck and J. Saxl, On the orders of maximal subgroups of the finite exceptional groups of Lie type. Proc. London Math. Soc. (3) 55 (1987), 299-330. Zbl 0627.20026 MR 896223

[Q] D. Quillen, Homotopy properties of the poset of nontrivial $p$-subgroups of a group. Adv. in Math. 28 (1978), 101-128. Zbl 0388.55007 MR 493916

[S] Y. Segev, On the uniqueness of Fischer's Baby Monster. Proc. London Math. Soc. (3) 62 (1991), 509-536. Zbl 0733.20007 MR 1095231

[Se] G. M. Seitz, Unipotent elements, tilting modules, and saturation. Invent. Math. 141 (2000), 467-502. Zbl 1053.20043 MR 1779618

[Si] S. Sidki, Some commutation patterns between involutions of a finite group. II. J. Algebra 39 (1976), 66-74. Zbl 0379.20029 MR 0414708

Received November 3, 2006; revised March 6, 2007

M. Aschbacher, California Institute of Technology, Pasadena, CA 91125, U.S.A.

E-mail: asch@its.caltech.edu

R. Guralnick, Department of Mathematics, University of Southern California, Los Angeles, CA 90089-2532, U.S.A.

E-mail: guralnic@usc.edu

Y. Segev, Department of Mathematics, Ben-Gurion University, Beer-Sheva 84105, Israel

E-mail: yoavs@math.bgu.ac.il 